\title{
Delinquenz und Kriminalität als soziales Problem (Draft)
}

\author{
Dietrich Oberwittler
}

erscheint in:

Albrecht, Günter / Groenemeyer, Axel (Hg.), 2011:

Handbuch soziale Probleme (2. Auflage). Wiesbaden: VS Verlag für Sozialwissenschaften

(ISBN: 978-3-531-32117-2) 


\title{
Kriminalität und Delinquenz als soziales Problem
}

\author{
Dietrich Oberwittler
}

\section{Einleitung}

Die Spannbreite der Erfahrungen und Perspektiven, die sich mit den Begriffen Kriminalität und Delinquenz verbinden, ist enorm. Bankraub gehört ebenso zu dazu wie Insidertrading, körperliche Züchtigung der eigenen Kinder gleichermaßen wie Partnertötung, Ladendiebstahl ebenso wie Anlagebetrug, Schuleschwänzen, Schutzgelderpressung und Haschischkonsum. Kriminalität ist konkrete Erfahrung und mediale Konstruktion, Normalität und Extreme, Wahlkampfthema und Berufsfeld. Für die, die als Handelnde oder Erleidende direkt in Kriminalität involviert sind, reichen die Erfahrungen von Nervenkitzel und Freude über Routine, Frustration, Alltagsärgernis und Lebensschicksal. Kalkuliert man die durch Kriminalität verursachten Schäden und rechnet die Ausgaben für Polizisten, Staatsanwälte, Richter und Gefängnisse dazu, so kommt jährlich ein hoher Milliardenbetrag zusammen. Summiert man andererseits den durch reale und fiktive Geschichten von Kriminalität erzielten Umsatz von Zeitungen, Buchverlagen und Privatsendern, so zeigt sich ebenfalls eine beachtliche ökonomische Bedeutung. Kriminalität qualifiziert sich sicherlich für einen Spitzenplatz in der Rangliste sozialer Probleme. Als etwas, das per definitionem gegen die soziale Ordnung und gegen Normen verstößt, ist Kriminalität und Delinquenz immer ein soziales Problem, gemessen an eben diesen Normen.

Da, wie schon Emile Durkheim (1982 [1895]) feststellte, die Existenz von sozialen Normen notwendigerweise auch die Abweichung von diesen Normen bedingt, und Kriminalität daher zur Normalität jeder Gesellschaft gehört, kann auch der Diskurs über dieses Problem auf eine sehr lange Geschichte zurückblicken. Die Definitionen von und Reaktionen auf Kriminalität unterliegen einem historischen Wandel, der von gesellschaftspolitischen Grundströmungen getragen wird (Garland 1990b, 1991). Die wissenschaftliche Beschäftigung mit Kriminalität und Delinquenz ist ebenso vielfältig und widersprüchlich wie die Phänomene selbst und lässt sich daher nur schwer überschauen und synthetisieren. Um das soziale Problem Kriminalität und Delinquenz in einem Überblicksartikel zu behandeln, ist also ein gewisses Maß an Vereinfachung und Weglassen unabdingbar. Allerdings haben viele der in diesem Handbuch behandelten sozialen Probleme häufig auch einen direkten Bezug zu Kriminalität, stellen gar spezifische Delikttypen dar $(\Rightarrow$ Bannenberg: Korruption und Wirtschaftskriminalität als soziales Problem; $\Rightarrow$ Groenemeyer: Drogen, Drogenkonsum und Drogenabhängigkeit; $\Rightarrow$ Müller/Schröttle: Gewalt gegen Frauen und Gewalt im

Ich danke Gerhard Spieß (Universität Konstanz) für die Bereitstellung von Daten aus dem Konstanzer Inventar Kriminalitätsentwicklung, Gaby Löffler für ihre Hilfen bei der Textfertigstellung und den Herausgebern für wertvolle Hinweise und Unterstützungen. 
Geschlechterverhältnis; $\Rightarrow$ Stallberg: Prostitution) oder greifen in der Bearbeitung theoretischer Konzepte und Methoden auf Beispiele aus dem Bereich Kriminalität und Delinquenz zurück $(\Rightarrow$ Kury/Obergfell-Fuchs: Evaluationsforschung; $\Rightarrow$ Peters: Soziale Kontrolle; $\Rightarrow$ Albrecht: Methodische Probleme der Erforschung sozialer Probleme).

Das Ziel dieses Beitrages ist es, einen Überblick über den theoretischen und empirischen Wissensstand zu den gesellschaftlichen Bedeutungen von Kriminalität und Delinquenz und über aktuelle Forschungsschwerpunkte zu geben.

\section{Definitionen von Kriminalität und Delinquenz}

Kriminalität ist kein naturgegebenes Phänomen und keine klar umrissene Menge von Verhaltensweisen, auch wenn man zwischen einem eher stabilen Kernbereich und eher unscharfen und wechselhaften Rändern unterscheiden kann. Das lateinische Ursprungswort „crimen“ (Anklage, Vorwurf, Verbrechen) weist bereits auf die juristische Definition, nach der Kriminalität Handlungen beschreibt, die gegen strafrechtliche Normen verstoßen und daher mit den Mitteln des Strafrechts sanktioniert werden können. So vielfältig und wandelbar die Erscheinungsformen von Kriminalität auch sein mögen, so haben sie doch stets gemeinsam, dass sie einen - den Handelnden in der Regel auch bewussten - Bruch von Verbotsnormen darstellen (Hess/Scheerer 2004).

Eine gängige Definition für Kriminalität lautet daher „potenziell kriminalisierbare“ oder ,potenziell strafbare Handlungen“. Damit wird auch auf die wichtige Tatsache hingewiesen, dass nicht alle kriminellen Handlungen tatsächlich geahndet werden. Der juristische Kern der Kriminalitätsdefinition verkürzt komplizierte und nuancierte moralische Bewertungen im Einzelfall notwendigerweise auf eine 0/1-Entscheidung: Entweder eine Handlung ist strafbar oder sie ist es nicht (Cohen 1988). Welche Handlungen und welche Täter aus der Gesamtmenge aller strafbaren Handlungen von der staatlichen Sozialkontrolle erfasst werden und welche nicht, ist ein wichtiger Gegenstand der Kriminalitätsforschung, die hierfür unter anderem die Begriffe des Dunkelfelds und des „,second code“ geschaffen hat.

Im alltäglichen Sprachgebrauch und in den Massenmedien wird unter „der Kriminalität" meistens die Gesamtmenge der bekanntgewordenen und in der Kriminalstatistik dokumentierten Straftaten verstanden. Der aus der Wissenschaft stammende Begriff Delinquenz ist demgegenüber weiter und weicher: Er bezeichnet strafbare und nicht strafbare Regelverstöße eher leichterer Art, wie sie vor allem für das Kinder- und Jugendalter typisch sind, so auch Fehlverhalten in der Schule und gegenüber den Eltern oder aggressives Verhalten unter Gleichaltrigen. In der angelsächsischen Kriminalitätsforschung ist der Begriff „,delinquency“ gleichbedeutend mit Kriminalität und Problemverhalten von Kindern und Jugendlichen (Sampson 2001).

Es war vor allem das Verdienst des Definitionsansatzes (labeling approach), das Bewusstsein für die gesellschaftliche Konstruktion von Kriminalität und die Wandelbarkeit dieser Konstruktionen geschärft zu haben (Frehsee/Löschper/Smaus 1997). Welche Verhaltensweisen durch Strafrechtsnormen als kriminell definiert werden, ist prinzipiell variabel und reflektiert, was vom Staat (und denjenigen Gruppen, die die politische Willensbildung 
dominieren) als besonders schutzwürdig angesehen wird. Klar ist nur, dass stets die schwersten Normverstöße als Kriminalität definiert werden, für die die schärfsten Sanktionen, eben die Kriminalstrafen, angemessen erscheinen. Hierbei besteht keine vollständige Übereinstimmung, aber doch eine enge Beziehung zwischen dem, was man in der Tradition Durkheims (1977) als „moralisches Kollektivbewusstsein“ oder als gesellschaftliche Normvorstellungen bezeichnen könnte, und den Strafgesetzen, insbesondere wenn diese eine hohe Legitimität beanspruchen wollen. Wandeln sich gesellschaftliche Normvorstellungen, vollziehen die Strafgesetze diese Veränderung meist (teils mit einiger Verzögerung) nach.

Beispiele für den historischen Wandel von Strafnormen gibt es viele: Während VerstöBe gegen traditionelle Sexualnormen wie etwa Homosexualität oder vor- und außereheliche Beziehungen im Laufe des 20. Jahrhunderts weitgehend entkriminalisiert wurden $(\Rightarrow$ Lautmann: Sexuelle Auffälligkeit - Perversion), kam es in anderen Bereichen zu einer Auswietung der Kriminalitätsdefinitionen, so zum Beispiel bei verschiedenen Formen der Gewalt entgegen der Liberalisierung von Sexualnormen auch der sexuellen Gewalt (Klimke/Lautmann 2006; Menzel/Peters 2003). Die in den letzten Jahren in Deutschland erfolgte Kriminalisierung der körperlichen Züchtigung von Kindern durch ihre Erziehungsberechtigten (Bussmann 2005) oder des so genannten „Stalkings“ (Voß/Hoffmann/Wondrak 2006) sowie der Vergewaltigung in der Ehe sind vorläufige Endpunkte erfolgreicher „Karrieren“ sozialer Probleme, hinter denen eine sinkende Toleranzschwelle gegenüber Gewalt und deren Problematisierung durch gesellschaftliche Gruppierungen (,Moralunternehmer“) stehen (Cohen 1988). Die Entwicklung der Kriminalitätsdefinitionen folgt in diesem Bereich einer langfristigen Tendenz, Verletzungen der individuellen Unversehrtheit durch körperliche und psychische Aggressionen strenger zu sanktionieren, während andererseits Verstöße gegen traditionelle Moralvorstellungen und religiöse oder soziale Symbole und Tabus (etwa Blasphemie oder Majestäts-/Staatsbeleidigung) kaum oder gar nicht mehr als kriminell wahrgenommen werden (Durkheim 1982; Reuband 2004).

Dass dies früher anders war und in einigen Ländern heute noch anders ist, verweist nach Hess/Scheerer $(1997,2004)$ auf den historischen Zusammenhang der „ursprünglichen“ Kriminalitätskonstruktion mit dem „Widerspruch zwischen Herrschendem und Beherrschten“. In einigen „primitiven“ Gesellschaften blieben gemeinschaftliche Strafen ausschließlich den Verstößen gegen religiöse Normen und Tabus vorbehalten, während die Sanktionierung eines Diebstahls oder gar Mordes als Privatangelegenheit der Betroffenen angesehen wurde, denen jedoch Wiedergutmachung oder auch Vergeltung zustand (Ranulf 1964: 160 f.). Die Bezeichnung eines schädigenden Verhaltens als Kriminalität bedingt in diesem Sinne, dass sich außer dem Opfer auch die Gemeinschaft betroffen fühlt und zur Bekräftigung der verletzten sozialen Werte eine kollektive Sanktion erwartet (Mead 1918).

Der Herrschaftscharakter von Kriminalitätsdefinitionen tritt wohl am stärksten in totalitären Staaten hervor. Totalitäre Staaten benutzen das Strafrecht zum Beispiel zur Unterdrückung der freien Meinungsbildung und -äußerung, weil diese eine existentielle Gefahr für sie darstellt. Dass jedoch das Hören feindlicher Rundfunksender im Nationalsozialismus, die Republikflucht aus der DDR oder die Veröffentlichung regierungskritischer Blogs im Internet in China von der jeweiligen Bevölkerung als kriminell angesehen wurde und wird, ist nicht anzunehmen. Die Legitimität, das heißt die freiwillige Anerkennung der Normen 
in der Bevölkerung, ist daher ein zentraler Aspekt der Gültigkeit von Kriminalitätskonstruktionen jenseits der formal-juristischen Definition (Tyler 1990).

Die gesellschaftskritisch ausgerichtete Kriminalitätsforschung hat ganz im Gegensatz zu Durkheim (Garland 1990b: 55) den Konflikt- und Herrschaftscharakter der Kriminalitätsdefinitionen hervorgehoben. Warum ist Sozialhilfebetrug kriminell, aber die Vernichtung von tausenden Arbeitsplätzen, möglicherweise noch unter Mitnahme von staatlichen Subventionen, nicht? Die juristischen Legaldefinitionen und gesellschaftlichen Wahrnehmungen dessen, was „kriminell“ ist oder nicht, sind offensichtlich nicht immer deckungsgleich: Erstere orientieren sich an der formalen Strafbarkeit, Letztere eher am gesellschaftlichen Schaden des Handelns.

Die enormen Schadenshöhen treiben im Bereich der Wirtschaftskriminalität, für den Edwin Sutherland (1945) den Begriff des „white-collar crime“" geprägt hat, die Bemühungen um eine Ausweitung der Kriminalitätsdefinitionen an. So sind z. B. Schmiergeldzahlungen an ausländische Amtsträger seit 1999, an Privatpersonen im Ausland erst seit 2002 strafbar und damit kriminell im juristischen Sinne $\Leftrightarrow \Rightarrow$ Bannenberg: Korruption und Wirtschaftskriminalität als soziales Problem). Spektakuläre Einzelfälle wie der 2006 bekannt gewordene „Siemens-Skandal“ mit einer Schadenshöhe von mehr als einer Milliarde Euro sind eine direkte Folgewirkung der sich verändernden Kriminalitätsdefinitionen. Auch der erfolglose Versuch der Strafverfolgungsbehörden, die Prämienzahlungen in Millionenhöhe an Manager im Zusammenhang mit der Zerschlagung des Mannesmann-Konzerns und dem Verkauf von Konzernteilen an die britische Telekommunikationsgesellschaft Vodafone juristisch als Untreue zu ahnden, illustriert die ungeklärten Grauzonen im Bereich der Wirtschaftskriminalität (Boers/Theile/Karliczek 2004).

Obwohl sich die Liste der Beispiele von Entkriminalisierungen und Neudefinitionen von Kriminalität noch verlängern ließe (z. B. für den Bereich der (il)legalen Drogen einschließlich des Tabakrauchens), besteht doch andererseits eine bemerkenswerte historische und kulturvergleichende Konstanz in dem Feld, das häufig als „klassische Kriminalität“ bezeichnet wird und das den zahlenmäßig größten Teil der registrierten Straftaten ausmacht: Diebstahl, Einbruch, Raub, Betrug, Körperverletzung, Brandstiftung, Erpressung, Vergewaltigung, Mord und Totschlag und weitere Delikte, über deren kriminellen Charakter ein weitgehender gesellschaftlicher Konsens besteht. Der italienische Kriminologe Raffaelo Garofalo (1891) hat mit seinem allerdings umstrittenen Begriff des „,natürlichen Verbrechens" als erster versucht, einen kulturübergreifenden Kernbereich von Kriminalität unabhängig vom Strafrecht zu benennen (Brodeur/Ouellet 2004). Eine moderne Version findet sich in Gottfredson und Hirschis Definition von Kriminalität als „acts of force or fraud undertaken in the pursuit of self-interest" (1990: 15). In der Tat stehen Handlungen von Individuen, die andere oder die Allgemeinheit schädigen, indem sie sich deren Eigentum gegen deren Willen aneignen (durch Diebstahl, Raub, Betrug, Unterschlagung etc.), in den meisten Gesellschaften unter Strafe. Ebenso weit verbreitet ist das Verbot von Handlungen, die andere gegen deren Willen durch physische Gewalt verletzen, töten oder deren Besitz beschädigen. Beides widerspricht den prosozialen Grundsätzen der Fairness und Reziprozität, die den Kitt von Gesellschaften bilden und offenbar sehr tief im menschlichen Sozialverhalten angelegt sind, zumindest innerhalb der eigenen Bezugsgruppe oder Ingroup (Diekmann 2008; Fehr/Gintis 2007). Darauf weisen auch Studien der experimentellen Spieltheo- 
rie hin, nach der die Verletzung dieser prosozialen Verhaltensregeln bei den Mitmenschen ein Bedürfnis nach Bestrafung auslöst (de Quervain et al 2004; Fehr/Gächter 2002; Gintis/Bowles/Boyd 2005; Gürerk/Irlenbusch/Rockenbach 2006).

Dem kann zu Recht entgegengehalten werden, dass gerade in kapitalistischen Gesellschaften materieller Erfolg durch Konkurrenz und Bereicherung auch auf Kosten anderer moralisch nicht als verwerflich angesehen, sondern im Gegenteil geradezu idealisiert werden. Eine wichtige kriminalsoziologische Traditionslinie sieht Kriminalität sogar als eine deviante Spiegelung dieser ,subterranean values“ der konventionellen Gesellschaft (Hagan et al. 1998; Matza 1990 [1964]; Matza/Sykes 1961; Messner/Rosenfeld 1994). Trotz der hehren Ideale von Moral und Fairness ist der Alltag menschlichen Zusammenlebens von Konflikten durchzogen, in denen Unfairness und bösartige Schädigungen nichts Ungewöhnliches sind und die dennoch nicht strafrechtlich relevant sind (Hanak 1987). Was den im kulturvergleichenden Quer- und Längsschnitt recht stabilen Kern der Kriminalitätsdefinitionen von ihren weiten Grauzonen unterscheidet, ist möglicherweise die besonders plumpe, unmittelbare, oftmals physische und damit eindeutiger erkenn- und benennbare Verletzung der sozialen Spielregeln. Die Wahrnehmung von Normverletzungen als kriminell und die damit oftmals verbundene Empörung gelingt desto besser, je einfacher die Handlungsmuster strukturiert sind: Ein Bankraub entspricht diesem kriminellen Muster eher als eine strafbare Verletzung der Börsenregeln, selbst wenn Letztere den größeren Schaden erzeugt.

Es bleibt festzuhalten, dass trotz der Vielfalt, Veränderbarkeit und teils auch des Konfliktcharakters von Kriminalitätsdefinitionen dennoch viel für die These spricht, dass die Verschaffung von Vorteilen auf Kosten anderer und unter Missachtung allgemein akzeptierter Spielregeln, die auf moralischen Grundbegriffen der Fairness und Reziprozität aufbauen, stets einen Kern von Kriminalität ausmacht. Zwar verbieten die Heterogenität und Unschärfe, die an den Rändern der Kriminalitätsdefinitionen erheblich zunehmen, verkürzte und eindimensionale Begriffsbildungen. Andererseits wäre es ebenso verkehrt, dem Kriminalitätsbegriff deswegen eine Beliebigkeit zu unterstellen, nach der je nach subjektiver Perspektive oder Interessenlage alles oder nichts kriminell sein könnte (z. B. Kunz 2004: 31). Insofern Strafrechtsnormen die Einhaltung der Spielregeln und des prosozialen Verhaltens fördern, schützen sie die Schwächeren und die Allgemeinheit vor den Stärkeren und Rücksichtslosen. Selbst das englische Strafrechtssystem des 18. Jahrhunderts, von der gesellschaftskritischen Kriminalitätsforschung als Prototyp einer blutigen Klassenjustiz herausgestrichen (Hay et al. 1975; Linebaugh 2003), bietet nicht nur Anschauungsmaterial für den Gegensatz von Herrschenden und Beherrschten, sondern ebenso für diese Schutzfunktion staatlicher Herrschaft gegenüber Konflikt- und Kriminalitätsopfern (King 2000, 2004).

\section{Häufigkeit und Trends der Kriminalität}

\subsection{Amtlich registrierte Kriminalität}

Indem strafbares Verhalten von den Instanzen der staatlichen Sozialkontrolle entdeckt, registriert und sanktioniert wird, entsteht zugleich das Material, aus dem Kriminalstatistiken gewonnen werden. Da sie also im Zuge staatlichen Handelns entstehen, gehören Kriminalstatistiken zu der Gattung der so genannten ,prozessproduzierten Daten“ und teilen deren 
Vor- und Nachteile mit anderen amtlichen Datenquellen wie Arbeitslosen- oder Steuerstatistiken (Meulemann 2003). Kurz gesagt liegt der Vorteil prozessproduzierter Daten darin, dass sie durch die systematische und fortwährende Tätigkeit der staatlichen Verwaltungsorgane entstehen und in aller Regel Vollerhebungen sind. Die Summe aller individuellen Fäl- le und Personen geht in einer Statistik der Massenphänomene auf, die in modernen Gesellschaften eine eigene soziale Realität darstellt und maßgeblich zur Wahrnehmung sozialer Probleme beiträgt. Ihr Nachteil ergibt sich jedoch aus demselben Umstand: Sie gibt die soziale Realität so wieder, wie sie aus der Perspektive staatlichen Handelns erscheint. Das soziale Problem Kriminalität stellt sich in der amtlichen Kriminalstatistik genau so dar, wie es durch das Handeln von Polizei und Justiz generiert wird, das wiederum von den Kriminalitätsopfern angetrieben wird.

Die wichtigste Schlussfolgerung, die daraus zu ziehen ist, besteht in der grundlegenden Erkenntnis, dass amtliche Kriminalstatistiken zunächst ein Abbild der Aktivitäten der staatlichen Kontrollorgane sind. Die Summe der amtlich registrieren Straftaten wird als „Hellfeld“ der Kriminalität bezeichnet - im Gegensatz zum „Dunkelfeld“, der Summe aller potenziell strafbarer Handlungen einschließlich der nicht amtlich registrierten strafbaren Handlungen. Ob und welche potenziell strafbaren Handlungen registriert werden, hängt von einer Vielzahl von Faktoren ab - vor allem vom Anzeigeverhalten der Opfer - und stellt einen wichtigen Forschungsgegenstand dar.

Dennoch werden amtliche Kriminalstatistiken häufig als brauchbares Abbild einer sozialen Realität, der „Verbrechenswirklichkeit“ verstanden. Das gilt nicht nur für einen gu- ten Teil der medial-öffentlichen Wahrnehmung, sondern auch für einen wichtigen Zweig der quantitativ-empirischen Kriminologie, für die Kriminalstatistiken eine zentrale Datenquelle darstellen (Pratt/Cullen 2005). Dahinter steht die Annahme, dass das Hellfeld der amtlich registrierten Kriminalität Aussagekraft für die Struktur und Entwicklung der Kriminalität insgesamt hat (wenn nicht beide Aspekte - wie häufig in der medialen Berichterstattung - gänzlich gleichgesetzt werden). Diese hinsichtlich ihres Erkenntniswertes optimistische Interpretation der amtlichen Kriminalstatistik begleitet ihre Geschichte seit ihrer Entstehung im frühen 19. Jahrhundert (Melchers 1992).

Die so genannten Moralstatistiker wie der belgische Astronom Adolphe Quetelet (1921 [1869]) benutzten die jährlichen Statistiken der Strafgerichte ebenso wie Daten zu Suiziden, unehelichen Geburten etc. als Barometer des „moralischen“ Zustandes einer Gesellschaft. Zur Rechtfertigung formulierte Quetelet das Gesetz der „konstanten Verhältnisse“ zwischen Hell- und Dunkelfeld der Kriminalität (1921: 18), das allerdings mehr als einhundert Jahre vor der Erfindung von Dunkelfeldbefragungen gar nicht überprüfbar und schon bei seinen Zeitgenossen umstritten war. So kritisierte bereits 1838 der preußische Chefstatistiker die Verwendung der gerichtlichen Statistik als Indikator der Kriminalitätsentwicklung in einer Streitschrift mit dem aussagekräftigen Titel „Über die Unzulässigkeit eines Schlusses auf Sitten-Verfall aus der Vermehrung der gerichtlichen Untersuchungen gegen jugendliche Verbrecher" (Hoffmann 1847 [1838]). Seine These, dass ein Anstieg der registrierten Jugendkriminaliät nicht auf ein verändertes Verhalten der Jugendlichen, sondern auf eine zunehmende Tendenz zur formellen Sozialkontrolle zurückzuführen sei, ist bis heute aktu- ell geblieben und stellt nur ein Beispiel für eine weit verbreitete kritische Haltung gegen- über der

amtlichen

Kriminalstatistik

dar. 
Kriminalstatistik ist ein Sammelbegriff für unterschiedliche Fall- und Personenzählungen, die auf den aufeinander folgenden Stufen des Strafrechtssystems stattfinden. Die wichtigsten Indikatoren dieser Zählungen in der Bundesrepublik Deutschland sind die Polizeiliche Kriminalstatistik (PKS) und die justizielle Strafverfolgungsstatistik (BMI/BMJ 2001; Heinz 1990, 2008a).

Die Polizeiliche Kriminalstatistik (PKS) bildet die unterste, breiteste Stufe und ist die bekannteste Statistik. In der PKS werden die polizeilich registrierten Straftaten und, soweit vorhanden und bekannt, Opfer und Tatverdächtige gezählt. Maßgeblich ist der polizeiliche Kenntnis- und Bewertungsstand bei Abgabe des Falles an die Staatsanwaltschaft. Spätere Korrekturen und veränderte Bewertungen - z. B. die Unschuld eines Tatverdächtigen oder die Umdefinition von Straftatsbeständen - bleiben hier unberücksichtigt. Aus den Zählungen werden verschiedene bevölkerungsbasierte Raten gebildet, die als zentrale Indikatoren der Kriminalitätslage und -entwicklung gelten.

Die Häufigkeitsziffer (HZ) misst die Rate der Straftaten bezogen auf 100.000 Einwohner des jeweiligen Gebietes, in dem sich die Tat ereignet hat. Die HZ ist ein Indikator für das Volumen und die Entwicklung der registrierten Kriminalität und wird u. a. auch für regionale Vergleiche herangezogen.

Die Tatverdächtigenbelastungsziffer (TVBZ) misst die Rate der tatverdächtigen Personen, ebenfalls bezogen auf 100.000 Einwohner des Gebietes, in dem sich die Tat ereignet hat. Definitionsgemäß ist dies nur bei aufgeklärten Straftaten möglich. Wegen der erheblichen Tätermobilität in die urbanen Zentren führt die Zählung am Tatort statt am Wohnort zu einer selten beachteten Überschätzung der „Kriminalitätsbelastung“ der städtischen Bevölkerung. Spezifische Belastungsziffern nach sozio-demographischen Merkmalen (Altersgruppen, Geschlecht, Nationalität) sollen Vergleiche der kriminellen Aktivität verschiedener Bevölkerungsgruppen ermöglichen. Seit 1984 werden Personen, die mehrmals innerhalb eines Jahres registriert werden, nur noch einmal gezählt (,Echttäterzählung“).

Auf den nächsten Stufen folgt nach der weniger aussagekräftigen Staatsanwaltschaftsstatistik, die eine Zählung der Verfahrenserledigungen enthält, die Strafverfolgungsstatistik (StVSt) mit einer nach Delikten, Alter, Geschlecht und Staatsangehörigkeit der Angeklag- ten differenzierten Zählung der gerichtlichen Entscheidungen und Sanktionsformen. Über die Anwendung der schärfsten Sanktionsform, der Freiheitsstrafe, berichtet schließlich die Strafvollzugsstatistik mit den Belegungszahlen der Gefängnisse.

Ein Vergleich dieser Statistiken zeigt eindrucksvoll, wie stark die registrierte Kriminalität von der polizeilichen Erfassung der Straftaten bis zur gerichtlichen Verurteilung zahlenmäßig schrumpft, indem auf jeder Stufe des Strafrechtssystems ein ganz erheblicher Teil der Taten bzw. Täter herausgefiltert wird. Für 5,5 Mio. registrierte Straftaten in Westdeutschland im Jahr 2004 wurden von der Polizei 1,9 Mio. Tatverdächtige ermittelt, von denen weniger als die Hälfte, 0,7 Mio., gerichtlich abgeurteilt wurden. 580.000 Tatverdächtige wurden verurteilt, ca. 60.000 von ihnen zu Freiheitsstrafen (BMI/BMJ 2006: 22). Bis zur Anwendung der härtesten Sanktionsform werden also 97 Prozent der ursprünglich Tatverdächtigen im Prozess der formalen Sozialkontrolle herausgefiltert. Selbst bei Tötungsdelikten ist ein starker Schrumpfungsprozess von der polizeilichen Registrierung bis zur rechtskräftigen Verurteilung feststellbar: Nur in einem Viertel bis einem Drittel aller polizeilich registrierten Tötungsdelikte enden die Verfahren mit der Verurteilung der Tatver- 
dächtigen wegen eines Tötungsdeliktes (BMI/BMJ 2006: 77). Betrachtet man nur vollendete Tötungsdelikte, liegt dieser Anteil bei weniger als der Hälfte. Ein maßgeblicher Grund für diesen Schwund liegt in der Tendenz der Polizei, Delikte im Zweifelsfall schwerer zu bewerten, was in der Folge durch nachträgliches „Herunterdefinieren“ durch Staatsanwaltschaft oder Gericht korrigiert wird. Aus einem versuchten Mord kann so z. B. eine gefährliche Körperverletzung werden. Der Grundsatz ,in dubio pro reo“ führt andererseits in vielen Fällen zu einer Unterschätzung der Deliktschwere auf der gerichtlichen Ebene. Dieses Beispiel illustriert nur eines von vielen ähnlichen Problemen der amtlichen Kriminalstatistik, die zur Vorsicht bei der Verwendung und Interpretation dieser Zahlen mahnen.

Mengenmäßig wird die registrierte Kriminalität in Deutschland (6,3 Mio. Straftaten im Jahr 2007) von Bereicherungsdelikten (Eigentums- und Vermögenskriminalität) dominiert. Einfacher Diebstahl (z. B. Ladendiebstahl), schwerer Diebstahl (z. B. Wohnungseinbruch) und Vermögens- und Fälschungsdelikte (z. B. Kreditkartenbetrug) sind mit je rund 20 Prozent aller Straftaten für mehr als die Hälfte aller Straftaten verantwortlich.

Abbildung 1: Entwicklung der Häufigkeitsziffer der polizeilich registrierten Kriminalität, Deutschland 1963-2009*

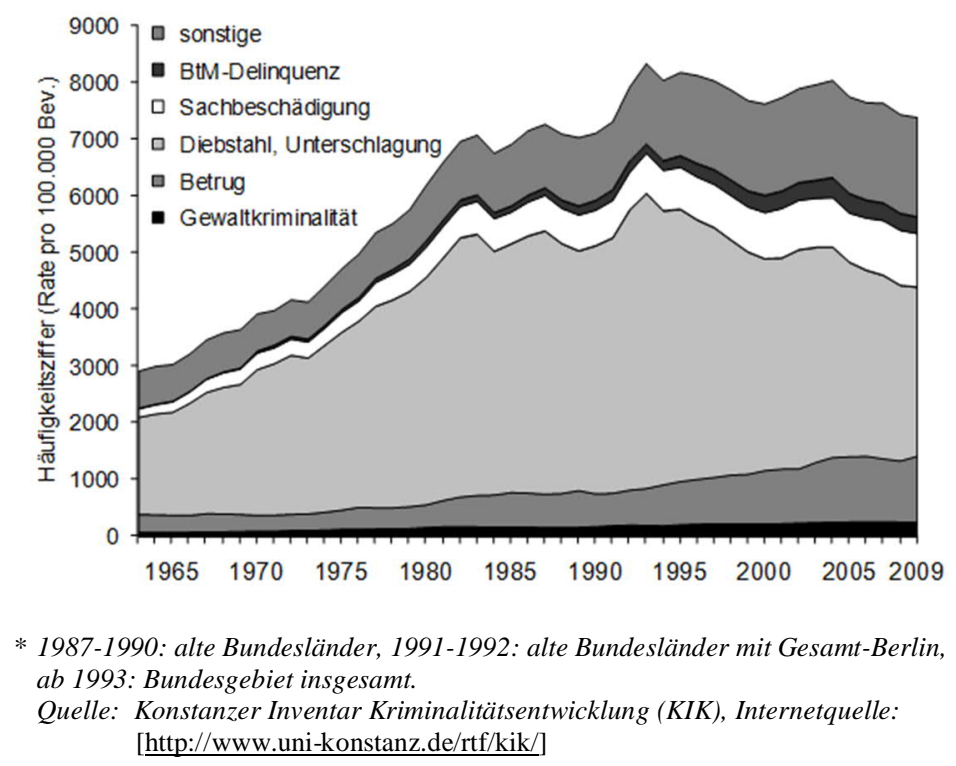

In den meisten Fällen ist der materielle Schaden gering. Der Schadenswert beträgt bei vier Fünfteln der einfachen Diebstahlsdelikte und bei zwei Dritteln der Betrugsdelikte maximal $250 €$. Dagegen entsteht in lediglich zwei Prozent der Fälle von Eigentums- und Vermögenskriminalität, die zum Bereich der Wirtschaftskriminalität gezählt werden, 50 Prozent des registrierten Gesamtschadens, nämlich 4,2 Milliarden $€$ im Jahr 2005 (BMI/BMJ 2006: 22). Während also die große Mehrzahl der Delikte Bagatellcharakter hat, richtet eine kleine 
Zahl von Straftaten im Bereich der „Weiße Kragen“-Kriminalität erheblichen materiellen Schaden an - und mit der öffentlichen Skandalisierung natürlich auch immateriellen Schaden etwa in Hinblick auf das Systemvertrauen der Bevölkerung.

$\mathrm{Zu}$ den frappierenden Entwicklungen in den meisten westlichen Industrieländern gehört der langfristige und steile Anstieg der Kriminalitätsraten nach dem Zweiten Weltkrieg auf das Zwei- bis Dreifache des Ausgangsniveaus. Dieser Trend dauerte in Deutschland in die die 1990er Jahre an (Abbildung 1) und machte laut David Garland (2001: 106) Kriminalität und die Furcht davor zu einer ,prominenten Tatsache des modernen Lebens“". Dass dieser Anstieg über weite Strecken mit Phasen wirtschaftlichen Wachstums zusammenfiel, hat die klassische Vorstellung des engen Zusammenhangs von Armut und Kriminalität nachhaltig erschüttert und die Entwicklung alternativer Erklärungsansätze - vor allem des Routine Activities Approach - provoziert (Cohen/Felson 1979, siehe Abschnitt 5.2).

Ein erheblicher Teil dieses Anstiegs geht auf „klassische“ Massendelikte wie Diebstahl und Unterschlagung zurück. In diesem Bereich ist jedoch seit Begin der 1990er Jahre eine Trendwende zu verzeichnen, die bis heute anhält. Ladendiebstähle sind seit ihrem Höchststand 1993 bis 2007 um 40 Prozent zurückgegangen. Schwerere Delikte wie Kfz-Diebstahl, Wohnungseinbrüche und Raubüberfälle auf Banken, Poststellen, Waren- und Werttransporte (diese zählen zur Gewaltkriminalität) sind sogar um 50 bis 60 Prozent seltener geworden (siehe Abbildung 2).

Abbildung 2: $\quad$ Entwicklung der Häufigkeitsziffer polizeilich registrierter Eigentumsdelikte und ihre prozentuale Veränderung, Deutschland 1987-2009"

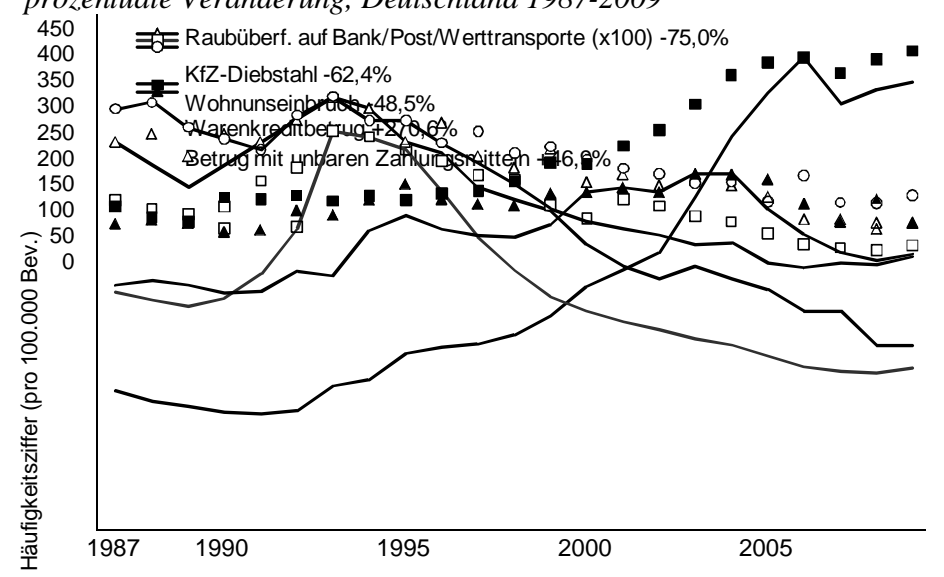

1987-1990: alte Bundesländer, 1991-1992: alte Bundesländer mit Gesamt-Berlin, ab 1993: Bundesgebiet insgesamt. Die Häufigkeitszahl für Raubüberfälle auf Bank/Post/Wertransporte wurde mit 100 multipliziert, da sie zu niedrig ist, um Entwicklungen im Maßstab der Tabelle darzustellen.

Quelle: Bundeskriminalamt, PKS-Zeitreihen 1987 bis 2009, eigene Berechnungen. Internetquelle: [http://www.bka.de/pks/zeitreihen/pdf/t01.pdf] 
Dagegen haben sich Betrugsdelikte in den vergangen zwei Jahrzehnten beinahe verdoppelt. In Abbildung 2 ist die besonders starke Zunahme des Warenkreditbetrugs, der vor allem im Kontext von Warenbestellungen und Ratenkäufen in Erscheinung tritt, um 250 Prozent zu erkennen. Diese Verschiebungen von traditionellen Formen der Bereicherungskriminalität, die quasi noch Handarbeit erforderten, zur eher an moderne Formen des Geschäftslebens angepassten Betrugskriminalität reflektiert möglicherweise den sozialen Wandel von der Industrie- zur Dienstleistungsgesellschaft mit veränderten Gelegenheitsstrukturen. Der Warenaustausch, die Zahlungswege und die Kommunikation insgesamt sind zunehmend weniger direkt organisiert, sondern werden in die Computernetze verlagert. Dadurch werden traditionelle Formen der Kriminalität weniger attraktiv und durch neue Formen wie z. B. Phishing ersetzt, die neu entstandende Gelegenheiten zur illegalen Bereicherung nutzen.

Die Gewaltkriminalität - darunter werden in der PKS Straftaten gegen das Leben, gegen die sexuelle Selbstbestimmung und Rohheitsdelikte wie Raub, Erpressung, Bedrohung und Körperverletzung (jedoch ohne das Massendelikt der leichten vorsätzlichen Körperverletzung) zusammengefasst - macht mit 3,5 Prozent nur einen sehr kleinen Teil der registrierten Kriminalität aus. Auch innerhalb der Gewaltkriminalität dominieren zahlenmäßig die weniger schweren Formen, während Sexual- und Tötungsdelikte in Deutschland sehr selten sind. Auch der deutliche Anstieg der registrierten Gewaltkriminalität in den letzten Jahren geht vor allem auf den Anstieg der weniger schwerwiegenden Delikte Körperverletzung und Raub zurück, während Tötungsdelikte und Vergewaltigung im zeitlichen Verlauf stabil bzw. rückläufig waren (Abbildung 3).

Abbildung 3: Entwicklung der Häufigkeitsziffer polizeilich registrierter Gewaltdelikte, Deutschland 1963-2009

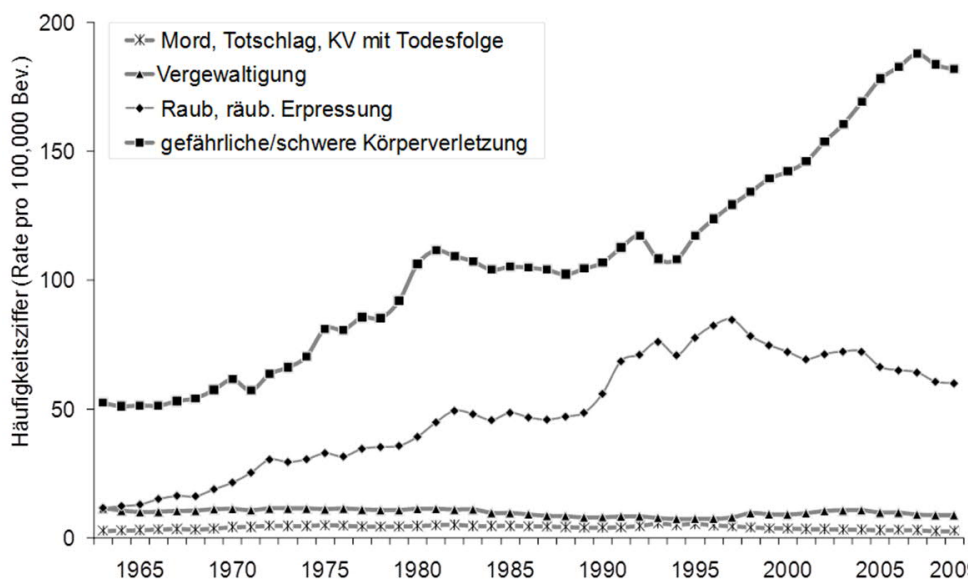

* 1987-1990: alte Bundesländer, 1991-1992: alte Bundesländer mit Gesamt-Berlin, ab 1993: Bundesgebiet insgesamt.

Quelle: Konstanzer Inventar Kriminalitätsentwicklung (KIK), Internetquelle: [http://www.uni-konstanz.de/rtf/kik/] 
Tötungsdelikte insgesamt sind seit der Mitte der 1990er Jahre um ca. 40 Prozent zurückgegangen, so dass Deutschland inzwischen zu den Ländern mit der niedrigsten Homizidrate in Europa gehört (BMI/BMJ 2006: 80). Auch Sexualmorde an Kindern und sexueller Missbrauch von Kindern werden entgegen der öffentlichen Wahrnehmung seit rund 30 Jahren kontinuierlich seltener registriert.

Im Gegensatz zu Bereicherungs- und Gewaltdelikten zeichnen sich verschiedene „opferlose“ Kriminalitätsformen dadurch aus, dass keine konkreten Opfer geschädigt werden. Ihre Verfolgung hängt daher weitgehend von den Aktivitäten der staatlichen Kontrollorga- ne (neben der Polizei auch des Zolls und anderer Behörden) ab. Besonders bedeutsam sind hier die Rauschgiftdelikte, die im Betäubungsmittelgesetz definiert werden. Die Zahl der registrierten Rauschgiftdelikte hat sich in den letzten 20 Jahren auf über 270.000 mehr als vervierfacht. Den größten Anteil (63\%) an der Rauschgiftkriminalität haben cannabisbezogene Delikte (BMI/BMJ 2006: 304).

Die in der PKS berechneten Tatverdächtigenbelastungsziffern (TVBZ) und deren zeitliche Entwicklung werden in der Öffentlichkeit als Gradmesser der „kriminellen Neigungen“ bestimmter Bevölkerungsgruppen wahrgenommen. Das für die bundesweite PKS verantwortliche Bundeskriminalamt verzichtet wegen methodischer Bedenken seit Längerem darauf, Kriminalitätsraten für Bevölkerungsgruppen mit nicht-deutscher Staatsangehörigkeit zu berechnen. Daher beziehen sich die folgenden Daten nur auf die deutsche Wohnbevölkerung.

Was dabei vor allem ins Auge sticht, sind die überdurchschnittlich hohen Belastungsziffern der männlichen Jugendlichen. Dem bekannten Muster der „Age-Crime“-Kurve folgend steigt die Kriminalitätsrate der männlichen Jugendlichen bis ca. 20 Jahre sehr steil an und fällt im Erwachsenenalter etwas flacher wieder ab (Abbildung 4).

2006 lag die TVBZ der männlichen Jugendlichen und Heranwachsenden mit deutscher Staatsangehörigkeit bei über 10.000. Das bedeutet, dass jährlich mehr als 10 Prozent aus dieser Gruppe polizeilich registriert werden; in den sozial benachteiligten Wohnquartieren der Großstädte erreicht dieser Anteil leicht 25 bis 30 Prozent. Nicht nur strafbares Verhalten, sondern auch dessen formelle Registrierung wird also zunehmend „normal“ für männliche Jugendliche. Die TVBZ der weiblichen Jugendlichen und Heranwachsenden liegt deutlich niedriger und ist in den letzten Jahren auch weniger stark angestiegen als die der männlichen, wenn man die absoluten Veränderungen der TVBZ betrachtet. Die oft geäuBerte Vermutung, dass Mädchen gegenüber den Jungen in Sachen Kriminalität „aufholen“, ist auf der Basis der registrierten Straftaten falsch.

Diese annähernde Verdoppelung der registrierten Jugendkriminalität in den 1990er Jahren, während die Kriminalitätsrate der erwachsenen Bevölkerung konstant geblieben ist, hat die gesellschaftliche Besorgnis über Jugendkriminalität immer wieder angeheizt und in der Kriminologie die Diskussion verstärkt, ob der Anstieg auf eine „,reale“ Zunahme des strafbaren Verhaltens oder auf eine Veränderung der sozialen Kontrolle von Jugendlichen zurückgeht. 
Abbildung 4: Tatverdächtigenbelastungsziffer (TVBZ) nach Altersgruppen und Geschlecht (nur Tatverdächtige mit deutscher Staatsangehörigkeit, Deutschland 2006)

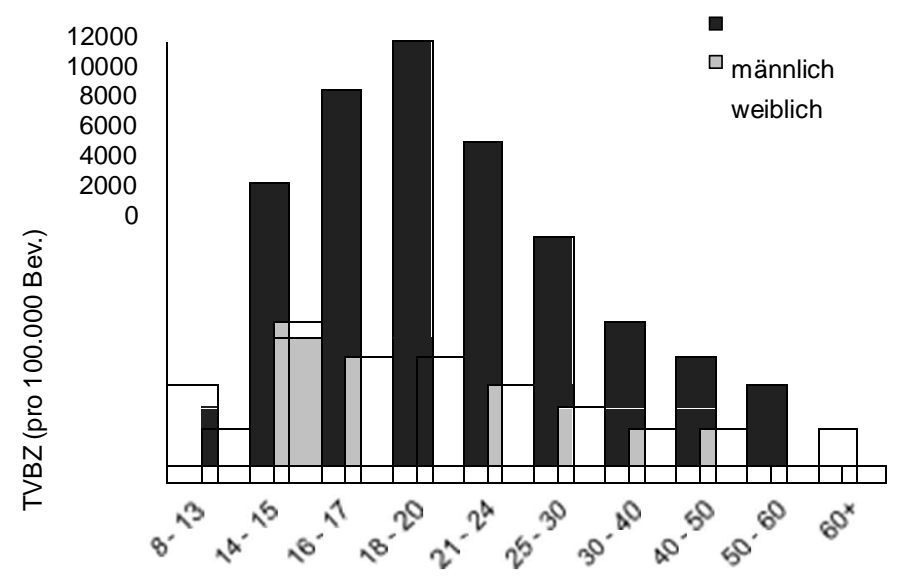

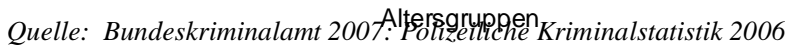

Abbildung 5: Entwicklung der Tatverdächtigenbelastungsziffern und Verurteiltenraten nach Altersgruppen in Deutschland 1984-2006*

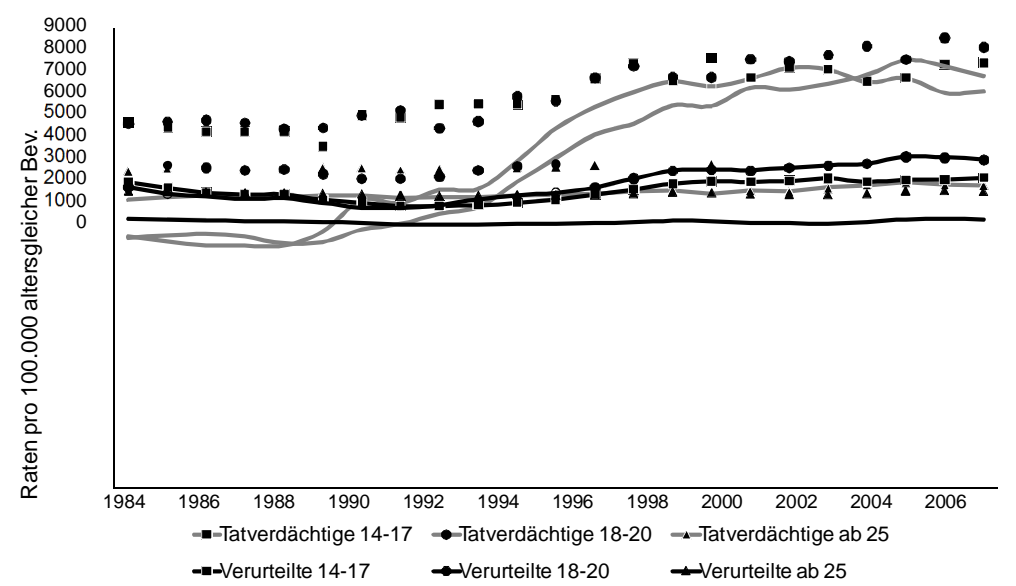

* 1987-1990: alte Bundesländer, 1991-1992: alte Bundesländer mit Gesamt-Berlin, ab 1993. Bundesgebiet insgesamt, nur deutsche Staatsangehörige.

Quelle: Konstanzer Inventar Kriminalitätsentwicklung (KIK), Internetquelle: [http:// www.uni-konstanz.de/rtf/kik/] 
Abbildung 5 zeigt, dass dem Anstieg der polizeilichen Registrierungen von Jugendlichen kein entsprechender Anstieg in den gerichtlichen Verurteilungen gefolgt ist. Die Erfassung von straffälligen Jugendlichen durch die staatliche Sozialkontrolle hat also stark zugenommen, jedoch haben sich die Behandlungs- und Sanktionsformen schon aus Kapazitätsgründen deutlich verändert, nämlich ins Vorfeld richterlicher Entscheidungen verlagert. Insofern dürften sich die Erfahrungen delinquenter Jugendlicher im System der staatlichen Sozialkontrolle in den letzten fünfzehn Jahren quantitativ und qualitativ verändert haben.

Der Anstieg der registrierten Jugendkriminalität ist nicht nur, aber zu einem bedeutsamen Teil auf die Gewalt- und BtM-Delikte zurückzuführen. In Abbildung 6 ist die Entwicklung der TVBZ jugendlicher Altersgruppen seit 1987 für vier Deliktsbereiche dargestellt.

Abbildung 6: $\quad$ Entwicklung der Tatverdächtigenbelastungsziffern einzelner Delikte nach Altersgruppen (nur deutsche Tatverdächtige, Deutschland 1987-2009)*
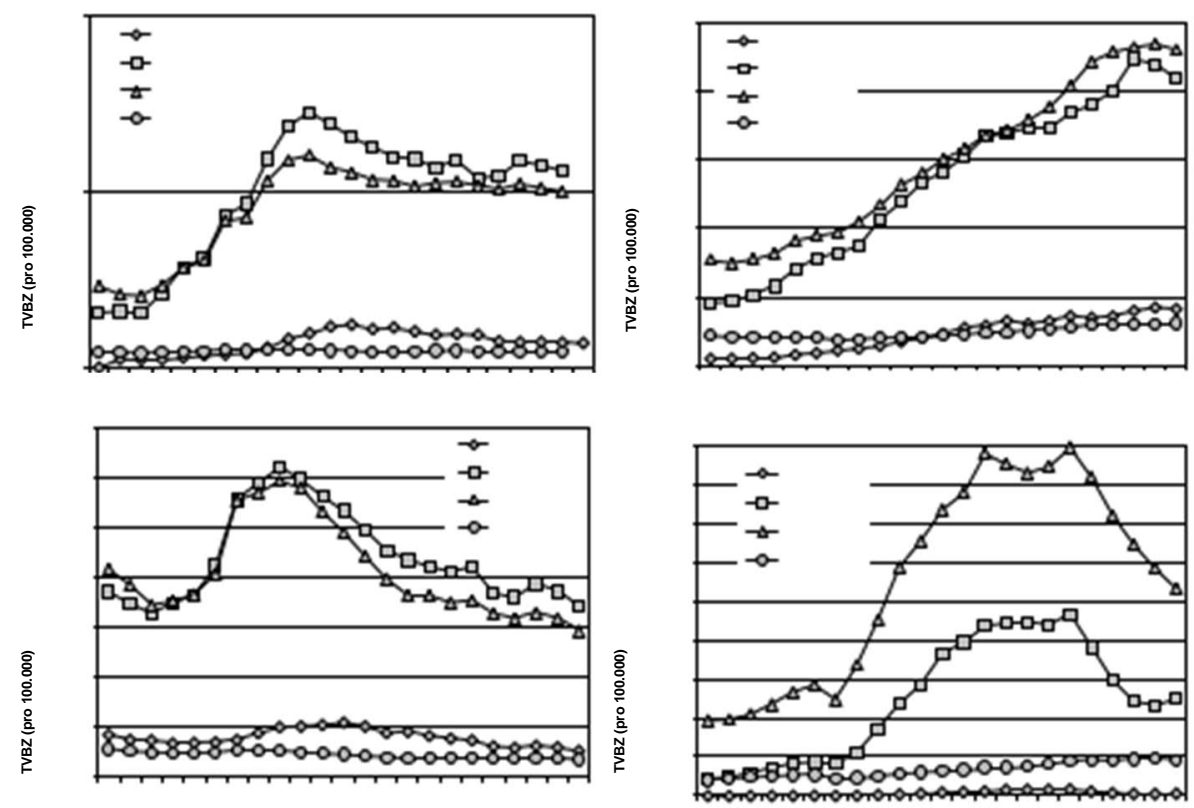

Quelle: Bundeskriminalamt, PKS-Zeitreihen 1987 bis 2009, Internetquelle: [http://www.bka.de/pks/zeitreihen/]

Während der starke Anstieg beim Raub und schweren Diebstahl seit dem Ende der 1990er Jahre gestoppt bzw. umgekehrt wurde, zeichnet sich bei den Körperverletzungsdelikten erst 
seit Kurzem ein Ende des Anstiegs ab, der in zwanzig Jahren zu einer Vervierfachung der Belastungsziffern geführt hat. Die TVBZ der BtM-Delikte, einem klassischen Kontrolldelikt, sind in den 1990er Jahren förmlich explodiert, dannach stagnieren sie auf hohem Niveau und fallen seit 2005 wieder deutlich ab.

\subsection{Dunkelfeldbefragungen}

Methodische Aspekte

Standardisierte Bevölkerungsbefragungen zu den persönlichen Erfahrungen mit Kriminali- tät wurden seit den 1970er Jahren zuerst in den USA durchgeführt und später dort wie auch in den Niederlanden und Großbritannien als regelmäßige nationale Opferbefragungen institutionalisiert (Lynch 2006; Zauberman 2008). Diese Befragungen ebenso wie Täterbefragungen zur selbstberichteten Delinquenz haben heute eine zentrale Position als Datenbasis der sozialwissenschaftlichen Kriminalitätsforschung. In der öffentlichen Wahrnehmung haben sie jedoch der PKS noch längst nicht den Rang abgelaufen, zumal in Deutschland, wo es bislang mit wenigen Ausnahmen bei lokal begrenzten Studien geblieben ist.

Opfer- und Täterbefragungen mit Zufallsstichproben aus der Wohnbevölkerung dienen primär dem Ziel, das Dunkelfeld der nicht polizeilich registrierten Straftaten aufzuhellen und damit eine von der staatlichen Sozialkontrolle unabhängige Informationsquelle zur Kriminalität zu schaffen. Ein unverzerrtes Abbild der Kriminalitätswirklichkeit können allerdings auch Dunkelfeldbefragungen nicht bieten, denn auch sie stellen eine - wenn auch anders und viel breiter angelegte - Konstruktion der sozialen Realität dar, die den spezifischen subjektiven Perspektiven der beteiligten Akteure folgt (Lynch/Addington 2007; Wetzels 1996).

Um selbst verübte oder erlittene Handlungen in Dunkelfeldbefragungen zu berichten, müssen die Befragten diese zunächst als kriminell oder potenziell strafbar einordnen, zudem müssen sie sich überhaupt an sie erinnern und bereit sein, ehrlich darüber Auskunft zu geben. Diese Bedingungen sind stets nur unvollkommen und annäherungsweise erfüllt. Vor allem in den unscharfen Grauzonen der Strafbarkeit von Handlungen, wie zum Beispiel bei Gewaltinteraktionen zwischen Jugendlichen oder den alltäglichen Schummeleien im Geschäftsleben, kann es letztlich keine objektive Messung der Kriminalität geben; vielmehr entscheiden hier die Wahrnehmung und die Interessen der Betroffenen, was kriminell ist und was nicht (Hanak/Stehr/Steinert 1989).

Dies betrifft in erster Linie das Feld der alltäglichen, eher trivialen Vorfälle; je schwerwiegender die Handlungen, desto eindeutiger die Zuschreibung der (potenziellen) Strafbarkeit, selbst wenn sie im Dunkelfeld verbleiben. Wieder andere, ernst zu nehmende Bereiche der Kriminalität liegen in einem so genannten „doppelten Dunkelfeld“, das selbst in Befragungen nur schwer aufgehellt werden kann. So nimmt man an, dass Opfer von Gewalt in Partnerbeziehungen ihre Erfahrungen eher verschweigen oder gar nicht als Straftat interpretieren, als wenn es sich um Gewalt durch Außenstehende handelt $(\Rightarrow$ Müller/Schröttle: Gewalt gegen Frauen und Gewalt im Geschlechterverhältnis). Die üblichen Opferbefragungen lassen Kriminalitätsformen, die sich gegen institutionelle Opfer richten (z. B. Ladendieb stahl oder Versicherungsbetrug) ebenso wie Kontrolldelikte (z. B. Drogenhandel) außer 
Acht. In jüngster Zeit werden ergänzend spezialisierte Opferbefragungen bei Firmen durchgeführt (Bussmann/PricewaterhouseCoopers 2007).

Bei Täterbefragungen spielt die Bereitschaft der Befragten zur Offenheit eine noch gröBere Rolle für die Validität der Selbstberichte. Während Jugendliche noch eher bereit sind, ehrlich über ihr strafbares Verhalten zu berichten, dürfte diese Bereitschaft bei Erwachsenen geringer sein, weswegen Täterbefragungen jenseits des Jugendalters selten sind (Junger-Tas/Marshall 1999; van der Heijden et al. 2000).

Die Qualität von Täter- wie Opferbefragungen steht und fällt mit der Qualität der erreichten Stichprobe, wobei sinkende Ausschöpfungsraten von 50 Prozent oder weniger Probleme bereiten (Schnell 1997; Stoop 2005). Besonders marginalisierte Bevölkerungsgruppen wie z. B. Obdachlose oder Konsumenten und Konsumentinnen harter Drogen, die (aus der Täter- oder Opferperspektive) besonders anfällig für Kriminalität sind, können in repräsentativen Bevölkerungsbefragungen nur schlecht erreicht werden. Das Gleiche gilt auch für ältere und pflegebedürftige Menschen zu Hause oder in Seniorenheimen und Pflegeeinrichtungen, deren Gewalterfahrungen durch Angehörige oder Pflegepersonal kaum aufzudecken sind (Greve/Hosser/Wetzels 1996; Görgen 2004a, 2004b). Dies sind nur einige Beispiele für Probleme, die einen „naiven Realismus“ (Wetzels 1996) hinsichtlich der Abbildqualität von Dunkelfeldbefragungen ausschließen.

Ebenso wenig ist es jedoch angebracht, angesichts dieser Probleme das Kind mit dem Bade auszuschütten und den Informationsgehalt von Dunkelfeldbefragungen grundsätzlich zu negieren. Ein gestohlenes Mobiltelefon bleibt ein gestohlenes Mobiltelefon, und über die Strafbarkeit dieses Diebstahls bestehen weder beim Opfer noch beim Täter Zweifel. Weite Bereiche krimineller Handlungen lassen keinen nennenswerten Interpretationsspielraum zu, und ihre Bewertung als potenziell strafbar ist auch im Zeitverlauf recht stabil. Opfer- und Täterbefragungen erlauben es daher in der Tat, erheblich realistischere Aussagen über das Ausmaß von Kriminalität zu machen als amtliche Kriminalstatistiken. Auch sprechen unvermeidbare Messfehler und Unschärfen nicht grundsätzlich gegen die Interpretation zeitlicher Trends als Indikator eines Anstiegs oder Rückgangs der Kriminalität im Dunkelfeld (Lynch 2006: 268; Robert 2009). Externe Validierungen von Opferbefragungen zu Gewalt mit Daten der Notfallmedizin in Großbritannien haben eine sehr hohe Übereinstimmung der zeitlichen Trends ergeben (Sivarajasingam et al. 2009). Darüber hinaus erlauben es Dunkelfeldbefragungen auch, die Prozesse und Selektionsmechanismen des Übergangs einer strafbaren Handlung vom Dunkel- ins Hellfeld zu untersuchen. Schließlich verknüpfen Dunkelfeldbefragungen Informationen über strafbare Handlungen mit einer Vielzahl von persönlichen und kontextuellen Variablen und ermöglichen damit Zusammenhangsanalysen, die auf der Basis amtlicher Kriminalitätsdaten ausgeschlossen sind.

\section{Ergebnisse von Opferbefragungen}

Die letzte umfangreichere nationale Opferbefragung in Deutschland wurde im Jahr 1997 durchgeführt (Heinz et al. 1998; für einen Überblick siehe Schneider 2007). Dabei gaben rund 20 Prozent der Befragten an, im Laufe eines Jahres Opfer eines von 13 abgefragten Delikte geworden zu sein. Frauen und Männer waren ungefähr gleich häufig betroffen, mit dem Alter sank das Opferrisiko deutlich von ca. 25 auf 10 Prozent ab. Zu den häufigsten Delikten gehörten Sachbeschädigungen am Pkw (5,7 \%), Fahrraddiebstahl (5,2\%) und Be- 
trug (4\%); Gewaltdelikte wurden mit 2 Prozent für Bedrohung und tätlichen Angriff, 1,2 Prozent für Raub und 0,3 Prozent für Vergewaltigung (einschließlich Versuch) deutlich seltener berichtet. Vom Diebstahl ihres Pkw waren 1 Prozent, von einem Wohnungseinbruch (einschließlich Versuch) 1,8 Prozent der Befragten betroffen.

Eine Schweizer Opferbefragung im Jahr 2000 ergab folgende, recht ähnliche Raten (Killias/Haymoz/Lamon 2007): Wohnungseinbruch 2,6 Prozent, Pkw-Diebstahl 0,4 Prozent, Fahrraddiebstahl 6,5 Prozent, Gewalt/Drohung 2,5 Prozent, Raub 0,6 Prozent. Im internationalen Vergleich liegen diese Zahlen eher am unteren Ende der Skala. Allerdings unterschätzen allgemeine Opferbefragungen insbesondere Gewalt im sozialen Nahraum erheblich und werden daher durch spezialisierte Dunkelfeldbefragungen ergänzt (Strauss/ Gelles/Steinmetz 2006). In einer deutschen Studie zur Gewalt gegen Frauen berichteten 1 Prozent der Befragten, im letzten Jahr Opfer sexueller Gewalt geworden zu sein; diese Rate steigt auf 12,8 Prozent, wenn alle Opfererfahrungen sexueller Gewalt ab dem 16. Lebensjahr berücksichtigt werden (Müller/Schröttle 2004, $\Rightarrow$ Müller/Schröttle: Gewalt gegen Frauen und Gewalt im Geschlechterverhältnis).

Da sich Opferbefragungen in der Regel auf die erwachsene Wohnbevölkerung konzentrieren, bleiben in ihnen auch die Erfahrungen von Kindern und Jugendlichen unterbelichtet. Hier zeigen Schulbefragungen, die in der Regel kombinierte Täter- und Opferbefragungen sind, dass Jugendliche bedeutend öfter mit Gewalt konfrontiert werden als Erwachsene. Verschiedene Studien ergaben Opferraten von ca. 5 bis 8 Prozent für Raub, über 10 Prozent für Körperverletzung und 5 Prozent für sexuelle Gewalt gegen Mädchen (Baier et al. 2006; Oberwitttler et al. 2001).

Durchschnittswerte der Opferwerdung verdecken erhebliche Ungleichverteilungen des Opferrisikos, deren Umfang und Ursachen in Deutschland nur wenig untersucht sind (Schneider 2007). Zum einen gibt es regionale und lokale Unterschiede im Opferrisiko, für deren Untersuchung es sehr großer Stichproben bedarf. Großstadtbewohner, und besonders Bewohner und Bewohnerinnen sozial benachteiligter Wohnquartiere, haben bei bestimmten Delikten wie Gewalt und Sachbeschädigung ein deutlich erhöhtes Viktimisierungsrisiko. Studien auf der Basis US-amerikanischer und britischer Opferbefragungen konnten dies detailliert zeigen (Lauritsen 2001; Lauritsen/White 2001; Tseloni 2000, 2006). Daneben hat die Opferforschung schon früh die Abhängigkeit des Opferrisikos vom Lebensstil herausgearbeitet: Wer einen aktiven, außerhäuslichen Lebensstil hat und insbesondere Kneipen und Vergnügungsviertel aufsucht, hat ein erheblich höheres Opferrisiko als andere Menschen (Arnold 1986; Hindelang/Gottfredson/Garofalo1978; Tseloni et al. 2004; Wittebrood/ Nieuwbeerta 1999).

Weitere, in Deutschland wenig erforschte Aspekte der ungleichen Risikoverteilung sind Mehrfachviktimisierungen und die Überlappung von Opfer- und Täterstatus. Nach den Ergebnissen des British Crime Survey konzentrieren sich ca. ein Drittel aller persönlichen Viktimisierungen auf lediglich 10 Prozent der Bevölkerung (Farrall/Pease 2007; Tseloni/ Pease 2005). Zudem wurde in vielen Studien festgestellt, dass Menschen, die selbst gegen Strafgesetze verstoßen, auch ein wesentlich höheres Opferrisiko tragen als gesetzestreue Menschen. Dies gilt sowohl für Gewaltdelikte bis hin zu Mord als auch für Eigentumsdelikte wie z.B. Wohnungseinbruch (Lauritsen/Laub 2007; Oberwittler/Wikström 2006). Für eine niederländische Kohorte von Straftätern lag das Risiko, später selbst durch ein Tö- 
tungsdelikt zu sterben, sechs mal höher als für die Normalbevölkerung. Eine von mehreren möglichen Erklärungen für dieses Phänomen baut auf der Subkulturtheorie auf, nach der Kriminalität in sozial marginalisierten Sozialmilieus konzentriert ist und zudem häufig den Charakter von Vergeltungsschlägen in konfliktreichen Interaktionen hat (Kubrin/Weitzer 2003b; Wolfgang/Ferracuti 1967, siehe Abschnitt 5.4).

\section{Reaktionen der Opfer auf Kriminalität}

Die Frage, welche Auswirkungen die Viktimisierung auf die Betroffenen haben, wie stark Opfer unter den Folgen einer Straftat leiden, wurde von der Forschung erst seit den 1980er Jahren im Zuge des Perspektivenwechsels vom Täter zum Opfer eingehender untersucht (Hassemer/Reemstma 2002; Kaiser/Kury/Albrecht 1991; Walklate 2007).

Die Opfererfahrungen sind ebenso vielfältig wie das Phänomen Kriminalität selbst, und die Konsequenzen hängen sowohl von der Schwere und Konstellationen der Tat als auch von den unterschiedlichen Ressourcen und Persönlichkeiten der Betroffenen ab, so dass Verallgemeinerungen nicht weiterführen. Das Ausmaß der persönlichen Betroffenheit lässt sich auch nicht ausschließlich in strafrechtlich relevanten Kriterien der Tatschwere bemessen, sondern hängt in vielen Fällen von anderen, nicht justiziablen Dimensionen ab. Hanak, Stehr und Steinert (1989) haben in einer ihrer offen angelegten qualitativen Studie zu „Ärgernissen und Lebenskatastrophen" herausgearbeitet, dass insbesondere länger andauernde Konflikt- und Opfererfahrungen in Partnerschaften und im sozialen Nahraum, die mit Ohnmacht und sozialer Isolation verbunden sind, weitreichende negative Konsequenzen tragen. In anderen Konstellationen, die zum Beispiel die erfolgreiche Mobilisierung sozialer Unterstützung ermöglichen, können dagegen auch schwere Straftaten unter Umständen gut verarbeitet werden. In ihren Interviews fanden Hanak et al. auch Beispiele, die sie wegen der erfolgreichen Verarbeitung und dem Gewinn an Lebenserfahrung und Selbstbehauptungskompetenz sogar als „Siegergeschichten“ bezeichnen, denen die Betroffenen auch positive Aspekte abgewinnen können (vgl. auch Aromaa 1991).

Opfererfahrungen können unter Umständen als Preis für riskante, aber nichtsdestotrotz lohnende Erlebnisse in Kauf genommen werden. Dieser überraschende Befund wird in ei- ner quantitativen Befragung von Gewaltopfern, die von der Opferhilfeorganisation „Der Weiße Ring" betreut wurden, bestätigt: 24 Prozent der Opfer berichteten über positive Auswirkungen der Viktimisierung auf ihre Lebensqualität vor allem in Hinblick auf die Erfahrung von Durchsetzungsfähigkeit und sozialer Unterstützung (Richter 1997: 127). Allerdings berichten dieselben Opfer ganz überwiegend über negative Auswirkungen auf das körperliche und seelische Befinden. Zwei Drittel der Befragten litten unter andauernden psychischen Beeinträchtigungen auch noch durchschnittlich drei Jahre nach der Tat; am schwersten waren diese Beeinträchtigungen bei Sexualdelikten. Wohnungseinbrüche führen zu einer ähnlich hohen psychischen Belastung wie Gewaltdelikte (Kilchling 1995: 157). Bei vielen Massendelikten wie Diebstahl oder Kfz-Sachbeschädigung stehen demgegenüber materielle Schäden im Vordergrund.

Die umfangreiche Forschung zur Kriminalitätsfurcht hat überwiegend keine eindeutigen und starken Effekte von Opfererfahrungen auf Kriminalitätsfurcht und Vermeidungsverhalten der Opfer erbracht (Boers 2001; Hale 1996). Jedoch gibt es durchaus Studien, die einen Furcht steigernden Einfluss von Opfererfahrungen aufgezeigt haben (Gabriel 1998; 
Kury/Obergfell-Fuchs/Würger 2000). Das Ausmaß der Furcht dürfte vor allem von der persönlichen Vulnerabilität der Betroffenen, die bei Frauen und älteren Menschen größer ist, sowie von einer erfolgreichen Verarbeitung der Opfererfahrung abhängen (Greve 1998; Killias 2002: 402; Schwind et al. 2001: 270).

Kriminalitätsopfer nehmen eine Schlüsselrolle im System der Sozialkontrolle ein, indem sie mit der Entscheidung für oder gegen eine Strafanzeige die grundsätzliche Richtung der Reaktionen auf eine strafbare Handlung festlegen: Polizeiliche Registrierung und eventuelle strafrechtliche Sanktionierung oder Verzicht auf staatliche Interventionen und eventuelle informelle Regelung oder Sanktionierung. In der 1997 durchgeführten nationalen Opferbefragung lagen die Anzeigeraten zwischen 20 Prozent bei sexueller Belästigung, 26 Prozent bei Betrug, 37 Prozent bei tätlichem Angriff, 57 Prozent bei einfachem Diebstahl und Sachbeschädigung und rund 90 Prozent bei PKW-Diebstahl und Einbruchsdiebstahl (Heinz et al. 1998: 2 ff.). Kury, Obergfell-Fuchs und Würger (2000: 340) stellten in einer lokalen Opferbefragung niedrigere Anzeigeraten fest, die z. B. bei Raub und Diebstahl um 30 Prozent und bei Betrug nur bei 5 Prozent lagen. Bei jugendlichen Opfern ist die Anzeigeneigung etwas niedriger, so liegt sie z. B. nach den Ergebnissen der MPI-Schulbefragung ca. 15 Prozent bei Körperverletzung und 28 Prozent bei Raub (Oberwittler et al. 2001). Jedoch gibt es hier Hinweise auf einen ansteigenden Trend.

Auch die Polizei hat einen häufig unterschätzten Ermessensspielraum bei der Bearbeitung von Strafanzeigen. Während die Polizei gerade im Bereich der alltäglichen Gewalt traditionell einen großen Teil der Anzeigen quasi unter den Tisch fallen ließ (Kürzinger 1978), könnte sich dies inzwischen geändert haben. Dass in sehr vielen Fällen keine Strafanzeige gestellt wird und der Fall damit im Dunkelfeld verbleibt, hat vielschichtige Hintergründe (Hanak/Stehr/Steinert 1989; Köllisch 2004, 2009). Neben der Schwereeinschätzung hängt es auch von der Verfügbarkeit informeller Konfliktregelungsmechanismen oder Sanktionen ab, die häufig dann bevorzugt werden, wenn Täter und Opfer sich bereits kennen. Strafanzeigen sind dagegen die Regel, wenn diese wie bei Versicherungsleistungen eine Voraussetzung für den Ausgleich materieller Schäden sind.

Während ein Verzicht auf formelle Strafverfolgung in vielen Fällen, so vor allem bei Bagatelldelikten von Jugendlichen, als angemessen erscheint, kann dies in anderen Konstellationen auch ein Ausdruck der Resignation und mangelnden Vertrauens in das Polizei sein oder auf Ausbreitung subkultureller Milieus hindeuten, in denen Kriminalitätsopfer Angst vor Repressalien haben oder aber Selbstjustiz und Vergeltung bevorzugen (Black 1983; Carr/Napolitano/Keating 2007; Kubrin/Weitzer 2003b; Pitts 2008). Eine Situation eskalierender Bandenkämpfe wie in US- oder lateinamerikanischen Städten findet sich in Deutschland sicherlich nicht, aber dennoch gibt es empirische Anhaltspunkte für die Wirksamkeit eines subkulturellen „Code of the street“ (Anderson 1999) auf das Anzeigeverhalten. So errechnete Köllisch (2004: 161), dass bei jugendlichen Opfern von Raub oder Erpressung mit der Schwere des Deliktes zwar zum einen die Anzeigeneigung zunimmt (von $13 \%$ bei einem Schaden von unter $10 €$ auf $33 \%$ bei einem Schaden von über $50 €$ ), jedoch gleichzeitig auch der Anteil der Opfer von 11 auf 24 Prozent wächst, die eine Anzeige aus Angst vor Repressalien unterlässt. Jugendliche Gewaltopfer, die selber häufig delinquent sind, tendieren wesentlich eher zur Selbstjustiz und haben ein geringeres Vertrauen in die Polizei (Köl- 
lisch 2004: 182). Diese Befunde dürften auf erwachsene Bevölkerungsgruppen übertragbar sein.

\section{Ergebnisse von Täterbefragungen}

Seit Beginn der 1990er Jahre haben Jugendsoziologen, Pädagogen und Kriminologen im deutschsprachigen Raum eine ganze Reihe von lokalen oder regionalen Schulbefragungen durchgeführt, die das Dunkelfeld der Jugendkriminalität weiter aufhellen konnten (Baier et al. 2009; Eisner/Manzoni/Ribeaud 2000; Holtappels et al. 1997; Lösel/Bliesener 2003; Oberwittler et al. 2001; Pfeiffer et al. 1998; Raithel/Mansel 2003; Tillmann et al. 1999; Wetzels et al. 2001; Wilmers et al. 2002). Einige dieser Befragungen wurden nach einigen Jahren teils mehrfach wiederholt (Baier 2008; Baier/Windzio 2008; Fuchs et al. 2005; Mansel/Hurrelmann 1998), andere Studien begleiten Jugendliche über mehrere Jahre (Boers/ Reinecke 2007; Schumann 2003a, 2003b), so dass trotz des Fehlens nationalrepräsentativer Befragungen auch einige Aussagen über zeitliche Entwicklungen möglich sind. Im Vergleich zu den zuvor eher üblichen haushaltsbasierten face-to-face Befragungen (z. B. Albrecht/Howe/Wolterhoff-Neetix 1988; Albrecht/Howe 1992; Villmow/Stephan 1983) zeichnen sich Schulbefragungen im Klassenverband durch wesentlich größere Stichproben, höhere Ausschöpfungsraten und vermutlich auch geringere Verzerrungseffekte der sozialen Erwünschtheit aus (Oberwittler/Naplava 2002; Naplava/Oberwittler 2002; Köllisch/Oberwittler 2004). Inzwischen wurden auch international vergleichende Schulbefragungen zur selbstberichteten Delinquenz durchgeführt (Junger-Tas et al. 2010, vgl. Aebi 2009; Zauberman 2009).

Die Schulbefragungen haben bisherige grundlegende Erkenntnisse über die Erscheinungsformen der Jugenddelinquenz bestätigt, die unter den drei Begriffspaaren Ubiquität/Normalität, Spontanbewährung/Abbruch und Intensität/Konzentration zusammengefasst werden können (Boers 2008; Walter 2001). Mit Ubiquität ist gemeint, dass strafbare Verhaltensweisen im Jugendalter sehr weit verbreitet und insofern etwas Normales sind. Mit etwa 14 Jahren, also bei Beginn der Strafmündigkeit, begehen ca. 40 bis 50 Prozent aller (städtischen) Jugendlichen mindestens ein strafbares Delikt im Jahr - Schwarzfahren und illegale Kopien oder Downloads nicht mitgerechnet (Tabelle 1).

Beim Eintritt in das Erwachsenenalter sind dann Jugendliche, die in ihrem Leben noch gar nicht gegen Strafgesetze verstoßen haben, deutlich in der Minderheit. Je nach Studie liegt die Prävalenzrate (der Prozentanteil der Befragten, die ein oder mehrere Delikte angeben) für Ladendiebstahl bei 15 bis 30 Prozent, für Gewaltdelikte, unter denen die Körperverletzung ohne Waffen dominiert, um 20 Prozent. Schwere Delikte wie Wohnungseinbruch, Raub, Diebstahl aus Kfz oder von Motorrollern sind mit Prävalenzraten von rund 3 bis 6 Prozent weitaus seltener als Bagatelldelikte. Der Anteil der delinquenten Jungen liegt in allen Studien deutlich höher als der der Mädchen.

Die überwiegende Mehrheit der Delikte von Jugendlichen erreicht die Instanzen der staatlichen Sozialkontrolle nicht und bleibt damit im Dunkelfeld verborgen. In der MPISchulbefragung gaben nur 10 Prozent der Täter, die einen Raub begangen hatten, einen Polizeikontakt an; bei Fahrraddiebstahl waren es nur 5 Prozent, bei Ladendiebstahl immerhin 25 Prozent (Köllisch 2004: 218). Dennoch, und dies ist eine für die Jugendkriminalpolitik besonders wichtige Erkenntnis, kommt es schon im Laufe des Jugendalters bei vielen 
Jugendlichen zur so genannten Spontanbewährung, d. h. die Neigung zur Delinquenz geht deutlich zurück, ohne dass es einer formellen Sanktionierung oder Intervention bedarf.

Tabelle 1: Prävalenzraten* der selbstberichteten Delinquenz von Jugendlichen (Schüler und Schülerinnen in der 9. Jahrgangsstufe)

\begin{tabular}{lccc} 
& $\begin{array}{c}\text { Köln/Freiburg } \\
1999 / 2000^{\mathrm{a}}\end{array}$ & $\begin{array}{c}\text { Duisburg } \\
2004^{\mathrm{b}}\end{array}$ & $\begin{array}{c}\text { KFN } \\
2005 / 06^{\mathrm{c}}\end{array}$ \\
\hline Gesamtdelinquenz & 54 & 43 & - \\
Ladendiebstahl & 31 & 15 & 17 \\
Einbruch & 4 & 3 & 4 \\
Gewalt & 22 & 16 & 17 \\
Körperverletzung & 17 & 14 & 16 \\
Raub & 6 & 3 & 3
\end{tabular}

* Prozentanteil der befragten Schüler/innen, die im letzten Jahr ein oder mehrere Delikte begangen haben.

Quellen: a MPI-Schulbefragung 1999/2000 (Oberwittler et al. 2001, eigene Berechnungen);

${ }^{b}$ Projekt ,Jugendkriminalität in der Modernen Stadt' (Boers/Walburg/Reinecke 2006); ${ }^{c}$ KFN-

Schülerbefragung 2005 in Hannover, München, Stuttgart und Schwäbisch Gmünd (Einbruch: nur München/Hannover) (Baier 2008).

In der Panelbefragung von Duisburger Jugendlichen vom 13. bis zum 18. Lebensjahr liegt der Gipfel der Delinquenz bereits im Alter von 14 bis 15 Jahren, anschließend sinken die jährlichen Prävalenzraten wieder ab (Abbildung 7).

Abbildung 7: Entwicklungsverläufe der selbstberichteten Gewalt- und Eigentumsdelinquenz nach Geschlecht, 13. bis 18. Lebensjahr (Jahresprävalenzen, Duisburg 2002 bis 2007, Panelstudie „Kriminalität in der modernen Stadt“)
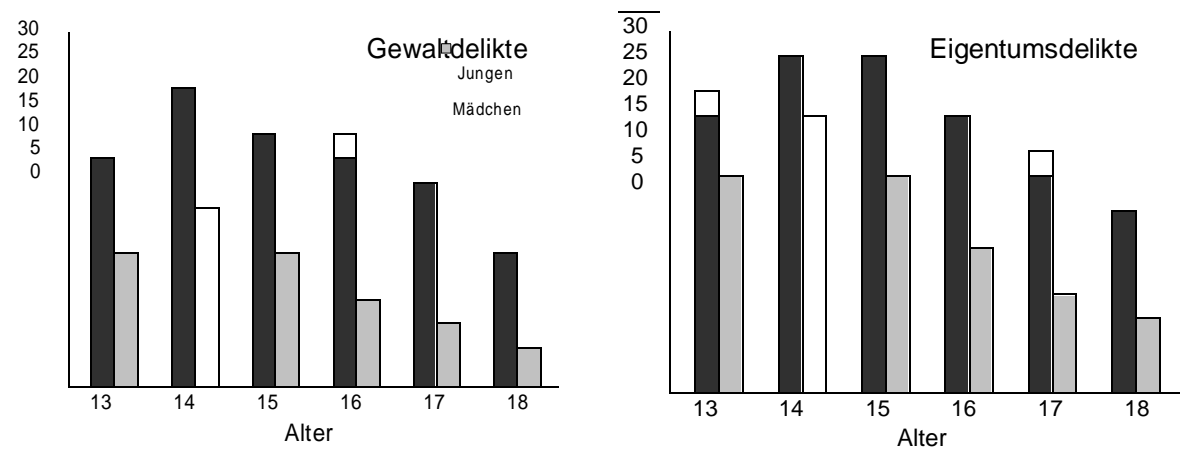

Quelle: Boers 2008: 346 
Der Altersgipfel der selbstberichteten Straffälligkeit liegt bei den Jungen demnach viel früher, als es aufgrund der polizeilich registrierten Jugendkriminalität erscheint; bei den Mädchen allerdings stimmen die „Age-Crime“-Kurven im Hell- und Dunkelfeld eher überein. Bei diesem akzentuierten Altersverlauf der Kriminalität mit dem Höhepunkt im Jugendalter handelt es sich um ein gesellschafts- und kulturübergreifendes Phänomen, das delinquentes Verhalten geradezu konstituierenden Bestandteil des Jugendalters erscheinen lässt, gleich, ob man dies eher soziologisch oder anthropologisch erklären möchte (Eisner 2002).

Während diese Ergebnisse der Dunkelfeldforschung also eine gelassene Haltung gegenüber dem Thema Jugendkriminalität fördern, weisen die Begriffe „Intensität“ und „Konzentration" auf die eigentlich problematische Dimension hin: Es gibt eine kleine Minderheit von etwa 5 Prozent der Jugendlichen, die aufgrund der Häufigkeit und Intensität ihres strafbaren Verhaltens als „Intensivtäter“ bezeichnet werden. Diese relativ kleine Gruppe ist et- wa für die Hälfte aller Delikte von Jugendlichen und für die meisten schweren Delikte wie Raub, Einbruch oder Drogenhandel verantwortlich, wobei in der Regel keine besondere Spezialisierung entweder auf Gewalt- oder Eigentumsdelikte zu beobachten ist (Boers/ Walburg/Reinecke 2006, siehe Abschnitt 5.4).

Die Konzentration der Delikte auf diese kleine Gruppe ist in Abbildung 8 anhand der Daten der MPI-Schulbefragung illustriert, in der die Jugendlichen aufsteigend nach ihrem Anteil an der Gesamtheit aller Delikte nach der Häufigkeit der selbstberichteten Delinquenz angeordnet sind. Nach dieser Berechnung fallen 57 Prozent aller Taten im Dunkelfeld auf 5 Prozent der Jugendlichen, die am meisten Delikte begehen. Es ist vor allem diese relativ kleine Gruppe von Intensivtätern am obersten Ende dieser sehr ungleichen Verteilung, die Risikofaktoren in persönlichen, familiären, schulischen und sozialräumlichen Dimensionen aufweisen und auf die sich die Forschung ebenso wie kriminalpolitisches Handeln konzentrieren sollten. Diese Extremgruppe ist der Polizei überwiegend bekannt, da das Entdeckungsrisiko insbesondere bei Gewaltdelikten und Einbruchsdiebstahl mit der Häufigkeit der Taten bis auf 100 Prozent ansteigt und damit das Dunkelfeld gänzlich aufgehellt wird (Köllisch 2004: 221).

Dunkelfeldstudien zur selbstberichteten Delinquenz im Erwachsenenalter sind weitaus seltener, und empirisch gesichertes Wissen muss durch Vermutungen ergänzt oder ersetzt werden. Sicher scheint einerseits, dass mit dem Alter nicht nur die eher jugendtypischen Formen der Kriminalität wie Vandalismus, Körperverletzungen, Raub, Einbruch, Diebstahl etc., sondern auch die Gesamthäufigkeit strafbaren Verhaltens deutlich zurückgehen. Andererseits gewinnt der Bereich der Kriminalität zunehmend an Bedeutung, den Frehsee (1991) als „Abweichung der Angepassten“ bezeichnet hat, und der im Kern die illegale materielle Bereicherung durch den Verstoß gegen Regeln im Beruf oder privaten Leben, etwa im Bereich der Steuern, Sozialabgaben oder des Umweltschutzes, betrifft. Berufliche Laufbahnen schaffen erst die passenden Gelegenheiten. Da hier häufig die Allgemeinheit oder eher anonyme Institutionen wie Arbeitgeber, Finanzamt und Versicherungskonzerne geschädigt werden, ist das Dunkelfeld ganz besonders groß. 
Abbildung 8: Konzentration der Delikte im Dunkelfeld nach Intensität der selbstberichteten Delinquenz, (Lorenzkurve)

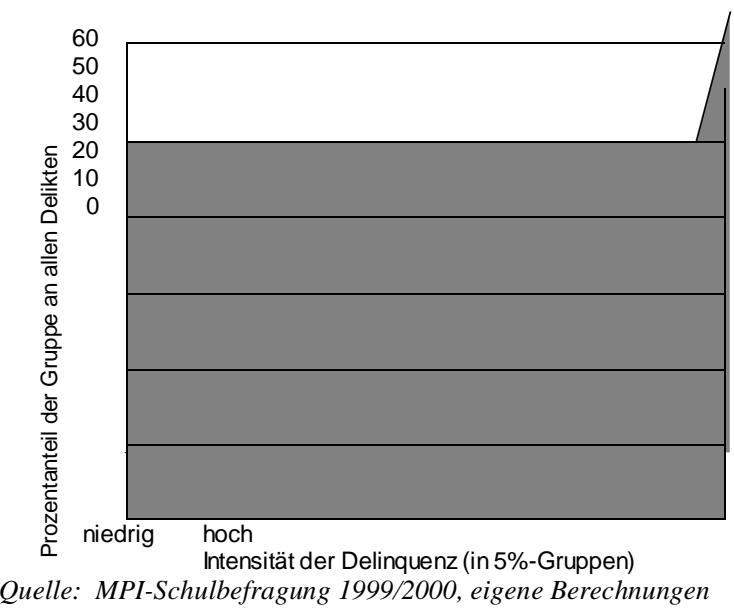

Karstedt und Farall (2006) führten hierzu eine Bevölkerungsbefragung in Großbritannien und Deutschland durch, bei der 70 Prozent der westdeutschen und 60 Prozent der britischen Befragten angaben, in ihrem Leben bereits eine von zehn Handlungen wie etwa überzogene Schadensmeldungen bei Versicherungen, fälschlichen Bezug von Sozialleistungen oder Vermeidung der Mehrwertsteuer begangen zu haben. Der Begriff der Ubiquität ist also auch zur Charakterisierung der Kriminalität im Erwachsenenalter angemessen, auch wenn die häufig mit großem materiellen Schaden verbundenen Normverstöße in der ,anständigen“ Mitte der Gesellschaft nicht im gleichen Maße wahrgenommen werden wie die Verfehlungen etwa von delinquenten Jugendlichen.

\subsection{Kriminalitättrends im Hell- und Dunkelfeld}

Ein maßgebliches Ziel von Dunkelfeldstudien ist es, zeitliche Veränderungen der Kriminalitätsbelastung unabhängig von der offiziell registrierten Kriminalität zu untersuchen und die Effekte des Anzeige- und Registrierungsverhaltens auf die Kriminalstatistik sowie de- ren mögliche Veränderungen im Zeitverlauf zu identifizieren. Insbesondere angesichts ei- nes lange andauernden Anstiegs der registrierten Jugendkriminalität lag das Erkenntnisinte- resse der meisten Forscher und Forscherinnen in den letzten Jahren darin, der amtlichen Kriminalstatistik etwas entgegenzusetzen und damit zur Entdramatisierung der öffentlichen Wahrnehmung beizutragen. Daher soll im Folgenden zunächst der Trend der Jugendkriminalität betrachtet werden.

In der Tat haben wiederholte Dunkelfeldbefragungen Belege dafür gefunden, dass die Häufigkeit delinquenten Verhaltens von Jugendlichen in der jüngsten Vergangenheit eher rückläufig ist. Fuchs et al. (2005) untersuchten die Entwicklung der Gewalt an bayerischen Schulen in den Jahren 1994, 1999 und 2004. Dabei stellten sie einen Rückgang der Gewalt 
an allen Schulformen insbesondere zwischen 1999 und 2004 fest, der an den Hauptschulen allerdings weniger ausgeprägt war als an Realschulen und Gymnasien.

Nach den wiederholten Schulbefragungen des KFN in mehreren deutschen Städten sank die Gewaltbelastung zwischen 1998 und 2005 aus der Täter- und Opferperspektive um ca. 10 bis 15 Prozent, jedoch ging die Gewalt am stärksten bei Gymnasiasten zurück, während bei Hauptschülern sogar ein weiterer Anstieg zu verzeichnen war (Baier 2008: 21). Rückläufig waren auch Sachbeschädigung und insbesondere Diebstahl. Die Duisburger Längsschnittstudie ergab einen Rückgang der selbstberichteten Gewalt- und Eigentumsdelikte zwischen 2002 und 2004 um sogar 20 bis 30 Prozent (Boers/Walburg/Reinecke 2006). Dieser rückläufige Trend scheint seit dem Ende der 1990er Jahre begonnen zu haben, während die noch spärlicheren Vergleichsbefragungen der 1980er und frühen 1990er Jahre zum Teil Hinweise auf eine ansteigende Gewaltneigung insbesondere von Mehrfachtätern ergeben hatten (Lösel/Bliesener/Averbeck 1998; Mansel/Hurrelmann 1998).

Eine weitere, sowohl von der polizeilichen Kriminalstatistik als auch von Dunkelfeldbefragungen unabhängige Informationsquelle unterstützt die Annahme vom Gewaltrückgang bei Jugendlichen: Die Versicherungsstatistik der so genannten „Raufunfälle“ in Schulen, in der alle ärztlich behandelten Verletzungen von Schülern und Schülerinnen erfasst werden, weist einen über viele Jahre anhaltenden rückläufigen Trend aus (Köllisch 2007).

Auch in national-repräsentativen Jugendbefragungen in den USA, Schweden und Finnland gehen die Dunkelfeldziffern aus der Täter- und Opferperspektive seit Mitte der 1990er Jahre zurück, so z. B. bei selbstberichtetem Diebstahl in Schweden von 66 auf 52 Prozent (Dinkes et al. 2007; Kivivouri 2007; Svensson/Ring 2007). Demgegenüber ist die Häufigkeit der Jugenddelinquenz im englischen Offending, Crime and Justice Survey in der jedoch kurzen Zeitspanne von 2004 bis 2006 konstant geblieben (Roe/Ashe 2008).

Entgegengesetzte Trends der Jugendgewalt in Dunkelfeldbefragungen und in der PKS wie in Deutschland legen den Verdacht nahe, dass mehr jugendliche Täter als früher bei der Polizei angezeigt und von dieser auch formell registriert werden. Diese Hypothese kann auf der Basis von Dunkelfeldbefragungen überprüft werden, die auch Fragen nach den erfolg- ten Reaktionen auf strafbare Handlungen stellen. In den KFN-Schulbefragungen zeigt sich dabei eine leichte, jedoch nicht signifikante Zunahme der Anzeigerate von jugendlichen Gewaltopfern zwischen 1998 und 2005, bei Raubdelikten fiel dieser Anstieg deutlicher aus (Baier 2008: 21). Ein Anstieg der Anzeigerate im gleichen Zeitraum fand sich auch in der Schweiz (Ribeaud/Eisner 2009). Der längste Zeitvergleich der Anzeigeraten bei Jugendgewalt anhand einer lokalen Wiederholungsstudie erstreckt sich über 25 Jahre (Köllisch/Oberwittler 2004; Oberwittler/Köllisch 2004). Während die Gewalthäufigkeit im Dunkelfeld zwischen 1983 und 1999 sogar leicht zurückging, wurde keiner der 1983 befragten Jugendlichen mit selbstberichteter Gewalt polizeilich registriert; 1999 war es dagegen jeder Dritte, und auch bei Diebstahlsdelikten erhöhte sich die Wahrscheinlichkeit einer polizeilichen Registrierung. Die Autoren interpretieren diese Entwicklung als Ausdruck eines grundlegenden sozialen Wandels, der den Umgang mit Problemverhalten von Kindern und Jugendlichen - nicht nur strafbares, sondern beispielsweise auch gesundheitsrelevantes Verhalten zunehmend aus der Hand der Familien und direkt Betroffenen in die Kompetenz von professionellen Instanzen verlagert. Andere mögliche Faktoren sind die Zunahme von interethnischen Täter-Opfer-Kons- 
tellationen, bei denen eine informelle Konfliktregelung schwerer fällt als in einer ethnisch homogenen Gesellschaft.

Zusammengefasst sprechen einige Indizien dafür, dass die scheinbar paradoxe Entwicklung der Jugendgewalt in Deutschland - Zunahme im Hellfeld, Rückgang im Dunkelfeld - durch Veränderungen im Anzeige- und Registrierungsverhalten erklärt werden kann. Dies unterstreicht einmal mehr die Bedeutung von regelmäßigen Dunkelfeldbefragungen für eine realistische Einschätzung von Kriminalitätstrends.

Allerdings sind Jugendliche schon aufgrund ihres geringen Bevölkerungsanteils von unter 10 Prozent nicht allein für die Kriminalitätsentwicklung insgesamt verantwortlich zu machen. Die Bochumer Opferbefragung von Schwind et al. (2001), die 1975, 1986 und 1998 durchgeführt wurde, ist die einzige deutsche Dunkelfeldstudie, die auch einige Aussagen über längerfristige Trends jenseits des Jugendalters erlaubt. Bei den Körperverletzungen zeigte sich über diesen Zeitraum keine nennenswerte Veränderung der Viktimisierungsrate, jedoch nahm auch bei den Erwachsenen der Anteil der Opfer deutlich $\mathrm{zu}$, die eine Anzeige erstatteten.

$\mathrm{Ob}$ sich diese punktuellen Indizien $\mathrm{zu}$ einem grundlegenden Trend verallgemeinern lassen, kann nur mit Hilfe der regelmäßigen nationalen Opferbefragungen in anderen Ländern entschieden werden. Für die Schweiz berichten Killias/Haymoz/Lamon (2007: 115) über stabile Anzeigeraten bei Körperverletzungsdelikten und sogar fallende Anzeigeraten bei Raub und Einbruch seit den 1980er Jahren. In England sind die im British Crime Survey gemessenen Anzeigeraten zwischen 1981 und 2006 dagegen bei verschiedenen Delikten deutlich angestiegen, so insbesondere bei Gewaltviktimisierungen durch Partner oder Bekannte, nicht jedoch durch Fremde, die von Beginn an höher lagen (Nicholas et al. 2007: 47). In den Niederlanden veränderte sich zwar das Anzeigeverhalten der Gewaltopfer zwischen 1980 und 2000 nicht deutlich, jedoch wurde ein wachsender Anteil dieser Anzeigen von der Polizei tatsächlich registriert (66\% im Jahr 1998 gegenüber nur $37 \%$ einige Jahre zuvor), unter anderem durch die Einführung von Computersystemen (Wittebrood/Junger 2002).

Das veränderte Verhalten der Polizei, nicht nur der Opfer, könnte demnach für einen Teil des Anstiegs der offiziellen Kriminalstatistik verantwortlich sein. 2002 sorgten in England neue Erfassungsregeln für die polizeiliche Registrierung von Delikten zu einem Anstieg der Kriminalstatistik um ca. 10 Prozent (Simmons/Legg/Hosking 2003). In einer de-

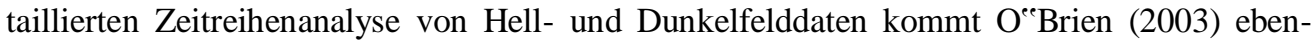
falls zu dem Schluss, dass der Anstieg der registrierten Gewaltkriminalität in den USA während der 1970er und 1980er Jahre in erster Linie auf eine gesteigerte Effizienz der polizeilichen Registrierung zurückzuführen ist. Die durch einen tatsächlichen Kriminalitätsanstieg in den 1960er Jahren mit angestoßene punitive Kriminalpolitik des ,get tough on cri-

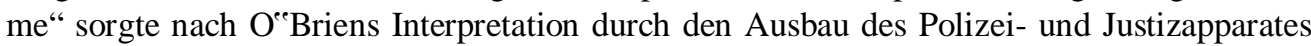
selbst dafür, dass die Kriminalstatistik auch in den darauffolgenden Jahrzehnten nach oben zeigte und sich damit für einige Zeit von den realen Trends abkoppelte (ebd.: 515).

Diese Befunde könnten den Eindruck verstärken, der bei vielen statistik-skeptischen Kriminalitätsforschern und -forscherinnen ohnehin dominant ist (z. B. Kunz 2004), dass Kriminalstatistiken kein brauchbarer Indikator einer wie auch immer definierten Kriminalitätswirklichkeit sind und daher auch keine validen Erkenntnisse über zeitliche Veränderun- 
gen ermöglichen. Dies ist insofern richtig, als amtliche Kriminalstatistiken am besten gemeinsam mit regelmäßig wiederholten Dunkelfeldbefragungen interpretiert werden sollten, weil dadurch das Verhältnis von potenziell strafbaren und tatsächlich durch die staatliche Sozialkontrolle registrierten Handlungen geschätzt und einige der Filtermechanismen zwischen diesen beiden Ebenen transparent gemacht werden können. Gerade die Entwicklung der letzten ca. fünfzehn bis zwanzig Jahre zeigt jedoch in einigen Bereichen, wie teilweise verblüffend ähnlich die Trends in Dunkelfeldbefragungen und in der amtlichen Kriminalstatistik verliefen. In England und den USA zeigt die Entwicklung bei den Eigentumsdelikten sowohl in der polizeilichen Kriminalstatistik als auch in den Opferbefragungen nach einem Höhepunkt in der Mitte der 1990er Jahre gleichermaßen und deutlich nach unten, im Dunkelfeld sogar um rund 50 Prozent (Catalano 2006; Nicholas/Kershaw/Walker 2007). Die verfügbaren Statistiken in vielen westlichen Ländern lassen keinen Zweifel an der Einschätzung zu, dass die „klassische“ Kriminalität im letzten Jahrzehnt kontinuierlich und deutlich im Rückzug begriffen ist.

\section{Wahrnehmungen von Kriminalität als soziales Problem}

Die gesellschaftliche Bedeutung von Kriminalität geht über die statistischen Zahlen und die konkrete Erfahrungen als Opfer oder Täter, die die meisten Menschen selbst selten oder nie machen, weit hinaus. Für die Konstituierung von Kriminalität als soziales Problem sind die Wahrnehmungen und Interpretationen des normverletzenden Verhaltens sowie die Reaktionen darauf maßgeblich. Ein ähnliches Kriminalitätsvolumen kann im einen Land (oder zu einer Zeit) mit Gelassenheit registriert oder gar ignoriert und im anderen Land (oder zu einer anderen Zeit) als Ausdruck einer bedrohlichen Krise interpretiert werden. Ein und dieselbe kriminelle Handlung kann höchst unterschiedliche Reaktionen bei den direkt Betroffenen, den Mitmenschen, der (medialen) Öffentlichkeit und den Instanzen der Sozialkontrolle auslösen, wie die vergleichbaren Fälle von Tötungsdelikten von Kindern durch Kin- der in Großbritannien und Norwegen exemplarisch gezeigt haben (Green 2007, 2008). Problemwahrnehmungen stellen daher unabhängig von den realen Ereignissen eigene soziale Fakten mit vielfältigen Bezügen und Auswirkungen dar, die von alltäglichen Verhaltensroutinen bis hin zur politischen Kultur reichen.

Warum sich Menschen und Gesellschaften in der Bewertung von Kriminalität und in den damit korrespondierenden Präferenzen für den Umgang mit abweichendem Verhalten unterscheiden, und ob und wie sich im Zeitverlauf die Problemwahrnehmungen und die Kriminalpolitik verändert haben, hat sich in den letzten Jahren unter dem Schlagwort der „punitiven Wende“ zu einem zentralen und kontrovers diskutierten Forschungsthema entwickelt. Kriminalität ist ein symbolträchtiges Phänomen, das mit anderen Problemen verknüpft, mit verschiedenen Besorgnissen aufgeladen werden kann und insofern auch das widerspiegelt, was Gesellschaften insgesamt bewegt und verunsichert. Dieser Beitrag konzentriert sich auf die Problemwahrnehmungen von Kriminalität, während die Bezüge zum kriminalpolitischen Handeln dem Themenfeld „Soziale Kontrolle“ zuzuordnen sind, auch wenn beide Aspekte miteinander verwoben sind ( $\Rightarrow$ Peters: Soziale Kontrolle). 
Im Folgenden werden zunächst einige elementare Bausteine oder Dimensionen vorgestellt, die in verschiedenen Gesellschaften in unterschiedlicher Weise bei der Konstruktion der sozialen Problemwahrnehmungen zusammenwirken. $\mathrm{Zu}$ diesen Bausteinen zähle ich grundlegende emotionale Reaktionen auf Normverletzungen, insbesondere Strafbedürfnisse und Furcht, Deutungsmuster von Kriminalität sowie die Rolle von Medien und Politik. Diese kollektiven sozialen Problemwahrnehmungen sind von den konkreten, individuellen Opfererfahrungen $\mathrm{zu}$ unterscheiden. Weiterhin ist es wichtig, die veröffentlichten Diskurse und Meinungen in den Massenmedien nicht mit den Alltagsvorstellungen der Menschen gleichzusetzen, auch wenn diese zweifelsohne von den Medien mit beeinflusst werden.

\subsection{Emotionen und Strafbedürfnisse}

Auf der elementarsten Ebene kann angenommen werden, dass Normverletzungen bei den Mitmenschen häufig negative emotionale Reaktionen auslösen (de Quervain et al. 2004; Fehr/Gächter 2002). Dies gilt, obwohl auch ambivalente oder sogar positive Bewertungen zur Realität des sozialen Problems Kriminalität gehören (Katz 1988). Bemerkenswert ist die Allgegenwart, mit der reale und erfundene Darstellungen von Kriminalität die Medienwelt durchziehen, und zwar nicht erst im Zeitalter der elektronischen Massenmedien (Hess/ Scheerer 1997: 134; Walter/Albrecht/Kania 2004). Die prominente Behandlung des Themas Kriminalität gerade in fiktionalen Genres hat einerseits mit der Faszination der Grenzüberschreitungen und Irrwegen menschlichen Handelns und den damit verbundenen Gefahren zu tun - vor allem wenn man selbst nicht davon betroffen ist -, andererseits wird mit den Kriminalitätsgeschichten eine Vergewisserung moralischer Prinzipien transportiert (Stehr 1998).

Die Rolle von starken Emotionen, von Empörung, Vergeltung, ja Rachegefühlen im System der Kriminalitätskontrolle wird in jüngster Zeit wieder neu diskutiert (Bieneck/Oswald/Hupfeld-Heinemeann 2009; Karstedt 2006, 2007a, 2007b; vgl. auch Fehr/Gintis 2007). Nach konventioneller Auffassung wurden diese Emotionen im Laufe der Zivilisierung, Rationalisierung und Bürokratisierung staatlicher Herrschaft immer weiter zugunsten eines vernünftigen Umgangs mit Kriminalität zurückgedrängt, grausame körperliche Strafen abgeschafft und Vergeltung als Strafziel durch Resozialisierung, Prävention und Wiedergutmachung überlagert oder ersetzt (Oswald et al. 2002; Sessar 1992). So sahen auch die Klassiker der Kriminalsoziologie wie Emile Durkheim (1992) und George Mead (1918) eine aggressive Feindseligkeit als die ,ursprüngliche“ Reaktionen der Gemeinschaft gegen Normabweichler an (vgl. dazu Jones/Scull 1992; Spitzer 1975; zusammenfassend Albrecht 1981, insbes. S. 334 ff.).

Dieses vereinfachende Modell wird jedoch der Komplexität einer Mehrzahl „,moralischer Gefühle“ (Karstedt 2002), die stets in soziale und kulturelle Kontexte eingebettet und damit auch wandelbar sind, nicht gerecht. Weder waren die Reaktionen auf Kriminalität in frühen historischen Epochen, wie von Durkheim angenommen, durchweg repressiv und grausam, sondern nahmen bereits moderne Elemente wie den Täter-Opfer-Ausgleich vorweg, noch verläuft die Entwicklung völlig gradlinig auf eine emotionsfreie und rein rationale, auf Nutzenüberlegungen konzentrierte Kriminalitätskontrolle zu. Vielmehr sind auch Wellen- oder Pendelbewegungen in den gesellschaftlichen Kriminalitätswahrnehmungen erkennbar, die seit den 1980er Jahren vorrangig in den USA und Großbritannien wieder zu 
einer Zunahme von expressiven und sozial ausgrenzenden Haltungen, zu einer neuen „Straflust“ (Cremer-Schäfer/Steinert 1998), geführt haben (Beckett 1997; Garland 2001).

Dennoch haben Emile Durkheim und nach ihm auch George Mead und Kai T. Erikson zutreffend elementare, über die unmittelbare Wirkung auf den Normbrecher hinausgehende gesellschaftliche Funktionen der negativ-punitiven Reaktionen auf Kriminalität beschrieben (vgl. Garland 1990a; Maruna/King 2004; Vidmar 2001): Indem die Gemeinschaft Normabweichungen sanktioniert, werden die Gültigkeit der zugrundeliegenden Normen bekräftigt und die Grenzen des tolerierbaren Verhaltens für alle aufgezeigt. Darüber hinaus wird durch die Abgrenzung von den Abweichlern die Zusammengehörigkeit der Gemeinschaft, das Kollektivbewusstsein (Durkheim), die Gruppensolidarität (Mead 1918: 580) oder die kulturelle Identität von Gemeinschaften (Erikson 1978: 13) gestärkt (vgl. die Kritik der Durkheimschen These der Funktionalität und Unvermeidbarkeit von Verbrechen bei Roshier 1977). Die marginalisierten und stigmatisierten Kriminellen sind in dieser funktionalistischen Perspektive zugespitzt formuliert das Opfer, das für den Zusammenhalt und die moralische Stabilität der Gesellschaft erbracht werden muss. Dieser Gedanke wird in der teils psychoanalytisch begründeten These der Sündenbock-Funktion von Kriminalität noch weitergetrieben, nach der durch die Ausgrenzung von Normbrechern die in allen Menschen vorhandenen (unterbewussten) Schuldgefühle von der Mehrheit abgespalten und auf einige wenige übertragen werden (Maruna/Matravers/King 2004).

Diese grundlegenden Mechanismen können in verschiedenen Gesellschaften in unterschiedlicher Weise bei der Herstellung gesellschaftlicher Reaktionen auf Kriminalität Verwendung finden. Sie weisen auf Zusammenhänge hin, ohne damit eine Zwangsläufigkeit zu unterstellen. Ein solcher elementarer Zusammenhang ist, dass zwischen den Problemwahrnehmungen von Kriminalität und der Wahrnehmung der sozialen Stabilität einer Gesellschaft insgesamt eine enge Beziehung besteht. Wird der gesellschaftliche Zusammenhalt als gefährdet angesehen, können damit eine sensiblere Problemwahrnehmung von Kriminalität und ein gesteigertes Strafbedürfnis einhergehen, welches der Bekräftigung von Gruppenidentitäten dient (Gabriel/Oswald 2007). Eine Studie des Sozialpsychologen Tom Tylers (Tyler/Boeckmann 1997) über Einstellungen zur US-amerikanischen Kriminalpolitik hat diese Verbindung von Strafbedürfnissen mit allgemeineren Wahrnehmungen sozialer Instabilität belegt, und ähnliche Befunde lieferte auch die Forschung zu Autoritarismus und politischem Konservativismus (Jost et al. 2003).

Zudem besteht eine Tendenz, die Problemwahrnehmungen eher auf solche Tätergruppen $\mathrm{zu}$ fokussieren, die ohnehin marginalisierte soziale Positionen einnehmen, wie etwa Angehörige ethnischer Minderheiten, denn diese eignen sich besonders gut für die negative Abgrenzung zwischen „Uns“ und „,den Anderen“, zwischen In-group und Out-group (Albrecht 1996). Die große Bedeutung des Themas „Ausländerkriminalität“ in der öffentlichen Wahrnehmung ist hierfür ein Beleg. Die historische Praxis der Lynch-Justiz in den amerikanischen Südstaaten, deren Häufigkeit mit der Agrarkonjunktur schwankte (Tolnay/Beck 1995), ist ein anderes, extremes Beispiel. Demgegenüber werden Normverletzungen in der gesellschaftlichen Mitte der „Angepassten“ wie Steuerhinterziehung oder Versicherungsbetrug eher bagatellisiert und aus der kollektiven Problemwahrnehmung ausgeblendet (Frehsee 1991: 37), es sei denn, bei den Tätern handelt es sich um prominente Manager oder Politiker

(Karstedt

2004). 


\subsection{Kriminalitätsfurcht}

Eine zentrale Rolle in der Diskussion über gesellschaftliche Problemwahrnehmungen hat die Kriminalitätsfurcht, die auch in Deutschland seit den 1990er Jahren vielfach empirisch untersucht wurde (Boers 1993, 2001, 2002; Gabriel/Greve 2003; Kury 2007; ObergfellFuchs/Kury 1996; Reuband 1992a, 1992b). Als in den 1970er und 1980er Jahren deutlich wurde, dass die in der Nachkriegszeit massiv angestiegenen Kriminalitätsraten chronischen Charakter haben, verlagerten sich zuerst in den USA die kriminalpolitischen Zielsetzungen gemeinsam mit dem Perspektivwechsel vom Täter zum Opfer auf eine positive Beeinflussung der subjektiven Auswirkungen von Kriminalität; die Herstellung eines Sicherheitsgefühls der Bevölkerung trat als eigenständiges Ziel neben die Herstellung von Sicherheit selbst (Boers 2001, 2002).

Unter Kriminalitätsfurcht werden persönliche Unsicherheitsgefühle und Risikoeinschätzungen - im Wesentlichen in Bezug auf Gewaltdelikte und Wohnungseinbruch - als auch damit verbundene Verhaltenskonsequenzen (Vermeidungsverhalten) verstanden. In Bevölkerungsbefragungen zeigt die subjektive Kriminalitätsfurcht nur schwache Verknüpfungen mit tatsächlichen Opfererfahrungen oder der messbaren Kriminalitätslage und -entwicklung, was zu einer breiten Diskussion über die so genannten „Kriminalitätsfurcht-Paradoxien" geführt hat. Das Furchtniveau der Bevölkerung erscheint erstens als wesentlich höher als durch die objektive Bedrohungslage gerechtfertigt. In der jüngsten internationalen Bevölkerungsbefragung zu Opfererfahrungen und Kriminalitätswahrnehmungen antworteten auf die so genannte Standardfrage zur Kriminalitätsfurcht, wie sicher man sich nach Einbruch der Dunkelheit in seiner Wohngegend fühlt, 30 Prozent der deutschen Befragten mit „etwas“ oder „,sehr unsicher“ (van Dijk/van Kesteren/Smit 2007: 131). Obwohl nur ein Prozent der Befragten im Jahr vor der Befragung Opfer eines Wohnungseinbruchs geworden waren, hielten es 23 Prozent für wahrscheinlich oder sehr wahrscheinlich, dass dies im darauffolgenden Jahr passieren könnte (ebd.: 127). Daneben zeigt sich in Befragungen immer wieder, dass die meisten Menschen den Anteil der Gewaltdelikte an der Gesamtkriminalität weit überschätzen und auch dann an einen erheblichen Kriminalitätsanstieg glauben, wenn die Kriminalstatistik über viele Jahre sinkende Fallzahlen verzeichnet (Pfeiffer/ Windzio/Kleimann 2004, 2005).

Zweitens zeigen Bevölkerungsgruppen mit einem besonders niedrigen Opferrisiko, nämlich Frauen und alte Menschen, die größte Furcht. Dieses Paradox wurde inzwischen durch die Hinzunahme psychologischer Konzepte wie Vulnerabilität, Coping-Ressourcen, Ängstlichkeit etc. zumindest teilweise aufgelöst; zudem zeigte sich, dass die Alterskurve der Kriminalitätsfurcht nicht linear ansteigt, sondern u-förmig verläuft, und auch junge Menschen - in Übereinstimmung mit ihrem Opferrisiko - mehr Angst vor Kriminalität haben (Gabriel/Greve 2003; Obergfell-Fuchs/Kury 1996). Andere Studien kritisierten die Messung der Kriminalitätsfurcht in standardisierten Befragungen und konnten zeigen, dass ein präziseres Nachfragen nach der Häufigkeit und Wertigkeit (Salienz) von erlebten Unsicherheitsgefühlen zu einer realistischeren, nämlich niedrigeren Einschätzung der persönlichen Kriminalitätsgefahren führen (Ditton/Farrall 2000; Farrall 2004; Farrall et al. 1997; Farrall/Jackson/Gray 2009; Jackson 2004a, 2004b, 2006).

Aus der Perspektive sozialer Probleme sind diejenigen Forschungsergebnisse besonders wichtig, die auf die große Bedeutung überindividueller Einflüsse auf unterschiedlichen 
sozialen Kontextebenen - vom unmittelbaren Wohnumfeld bis hin zu Nationalstaaten - auf Kriminalitätswahrnehmungen hinweisen, und die gleichzeitig die These belegen können, dass mit Kriminalitätsfurcht breitere soziale Ängste und Unsicherheitsgefühle transportiert werden (Hirtenlehner 2006, 2007; Jackson 2004b, 2006). Kriminalitätsfurcht variiert räumlich sehr stark und ist bei Bewohnern und Bewohnerinnen sozial benachteiligter Wohnquartiere in Großstädten am weitaus größten (Lagrange/Ferraro/Suprancic 1992; Lüdemann 2006; Oberwittler 2008; Sessar/Stangl/van Swaaningen 2007). Es sind sowohl die äußeren Anzeichen physischer und sozialer Unordnung im Straßenbild, in der Forschung als ,,incivilities“ und „disorder“ bezeichnet, als auch die hohen Konzentrationen armer und sozial marginalisierter Bevölkerungsgruppen, die mit Unsicherheitsgefühlen sehr eng korrelieren. Phänomene der Unordnung wie schlecht instand gehaltene Gebäude, Graffiti, Müll oder Gruppen von herumstehenden Jugendlichen bewirken vielleicht schon deswegen eine stärkere Verunsicherung als Kriminalität selbst, da sie mehr oder weniger dauerhaft präsent sind, während kriminelle Handlungen nur selten direkt beobachtbar sind (Häfele/Lüdemann 2006; Innes 2004; Lüdemann 2005; Perkins/Taylor 1996; Ross/Mirowsky/Prebesh 2001). Dies gilt, obwohl sich in der kriminologischen Forschung kaum Belege für einen kausalen Effekt von Disorder auf zunehmende Kriminalität im öffentlichen Raum finden ließen, der in der „Broken-Windows“-Theorie postuliert wurde (Markowitz et al. 2001; Robinson et al. 2003; Sampson/Raudenbush 1999; Skogan 1990; Taylor 2001; Wilson/Kelling 1982).

Disorder-Phänomene werden von vielen als sichtbare Zeichen urbanen Verfalls und schwindender Geltung sozialer Verhaltensregeln und sozialer Kontrolle interpretiert und verstärken vor allem bei Menschen mit geringen sozialen Ressourcen Vertrauensverluste und Ohnmachtsgefühle (Ross/Mirowsky/Prebesh 2001). Das Verhalten von Jugendlichen im öffentlichen Raum steht bei den Problemwahrnehmungen häufig an erster Stelle (Girling/Loader/Spark 2000; Oberwittler 2008). Die Symbolkraft des Jugendalters und insbesondere des Themas Jugendkriminalität für die Wahrnehmungen eines als bedrohlich empfundenen sozialen Wandels lässt sich historisch in wellenförmig wiederkehrenden „,moralischen Paniken“"weit zurückverfolgen (Cohen 1972; Pearson 1984; Trotha 1982).

Für eine Beziehung zwischen Kriminalitätsfurcht und generalisierten sozialen Ängsten sprechen auch auffallende nationale Unterschiede des Furchtniveaus zwischen den europäischen Ländern, wie sie sich zum Beispiel in den Ergebnissen des European Social Survey 2004 zeigten.

In Abbildung 9 ist die Rangfolge der Länder nach dem durchschnittlichen Ausmaß der Kriminalitätsfurcht, gemessen durch die Standardfrage nach dem Sicherheitsgefühl im Wohngebiet, graphisch dargestellt. Eine Mehrebenenanalyse unter Kontrolle der individuellen Erklärungsfaktoren wie Geschlecht, Bildungsstatus, Gemeindegröße etc. ergab, dass sich diese Länderunterschiede nicht durch Kriminalitätsbelastungen, sondern am besten durch das Ausmaß der wohlfahrtsstaatlichen Leistungen im Bereich der Kinder- und Familienhilfen erklären lassen, welche einen Schutz vor sozialen Risiken und sozialer Exklusion bieten und damit sozialen Unsicherheiten vorbeugen (Hummelsheim et al. 2010). 
Abbildung 9: Ländermittelwerte der Kriminalitätsfurcht in Europa (\%-Anteil der Befragten, die sich nachts in ihrem Wohngebiet ,unsicher" oder ,sehr unsicher" fühlen) (European Social Survey 2004-05)

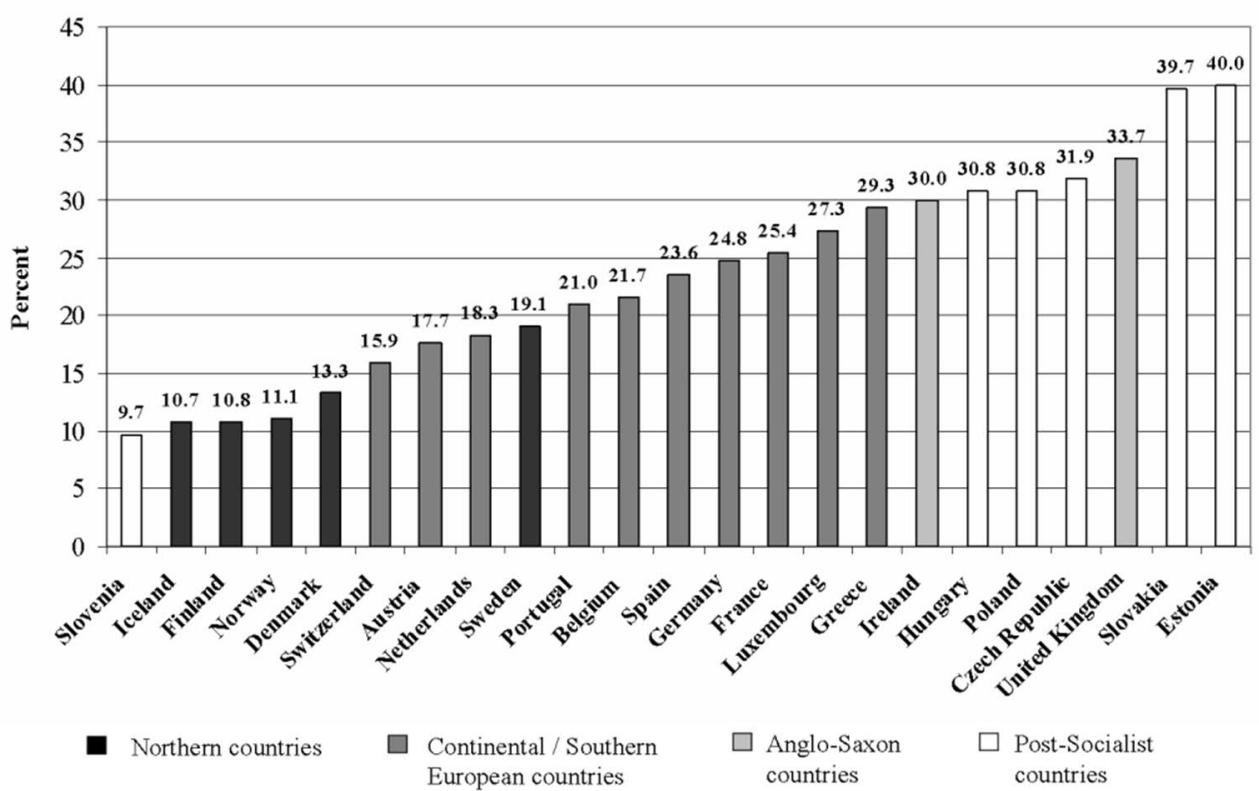

Quelle: Hummelsheim et al. 2010

Der starke vorübergehende Anstieg der Kriminalitätsfurcht in den neuen Bundesländern nach dem Ende der DDR wird ebenfalls als Beispiel einer Verknüpfung von Kriminalitätsund allgemeinen sozialen Ängsten in einer Situation schnellen sozialen Wandels interpretiert (Boers/Kurz 1997, 2001; Kury/Ferdinand 1999; Reuband 1996).

\subsection{Deutungsmuster der Kriminalität}

Kriminalität löst unvermeidlich die Frage nach dem Warum aus. Warum verstoßen Menschen gegen Strafgesetze, sind dafür persönliche oder gesellschaftliche Ursachen, und wenn ja, welche, verantwortlich? Die Antworten auf diese Fragen bilden unterschiedlich komplexe Deutungsmuster der Kriminalität sowohl in den wissenschaftlichen als auch in den populären, alltagsweltlichen Problemdiskursen. Auch wenn die wissenschaftlichen Diskurse differenzierter verlaufen, sind sie dennoch nicht völlig losgelöst von gesellschaftlichen Wahrnehmungen und spiegeln oftmals einen Zeitgeist wider. Die Interpretationen und Erklärungsansätze menschlichen Verhaltens sind eingebettet in übergeordnete Menschenbilder und Weltanschauungen, die dabei helfen, eine unüberschaubare Vielzahl nebeneinander existierender oder konkurrierender Einzelbilder und -erklärungen zu sinnhaften Mustern zu strukturieren (Garland 1990a: 193). Dabei spielen wohlfahrtstaatliche Einstellungen und die Frage, wie eine Gesellschaft mit ihren Randgruppen umgeht, eine zentrale Rolle. Hess und Scheerer (2004: 87; vgl. Becker 2007) sprechen von „Wahlverwandtschaften“ zwischen 
Menschenbildern, Erklärungsansätzen der Kriminalität und kriminalpolitischen Folgerun- gen.

Wer den Menschen als Produkt seiner sozialen Umwelt und Kriminelle als Opfer sozialer Missstände sieht, wird sozialpolitischen Maßnahmen den Vorrang vor Strafsanktionen geben; wer kriminelles Verhalten als Ausdruck pathologischer Persönlichkeitsstörungen betrachtet, befürwortet eher punitive und sozial ausschließende Reaktionen, die vor allem den Schutz der Gesellschaft vor Straftätern zum Ziel haben; wer dagegen Menschen für rationale Glücksmaximierer hält, die Kosten und Nutzen einer Straftat abwägen, sollte an die abschreckende Wirkung von Kontrollmaßnahmen und Strafandrohungen glauben (vgl. auch Groenemeyer 2001).

Diese holzschnittartige Verbindung unterschiedlicher Erklärungsmuster und kriminalpolitischer Konsequenzen stellt sich in der Realität komplexer und widersprüchlicher dar. Die psychologische Attributionsforschung begann mit der Unterscheidung von internen (auf das Individuum bezogenen) und externen (auf die Umwelt bezogenen) Zuschreibungen der Ursachen menschlichen (Fehl-)Verhaltens (Cullen et al. 1985; Hewstone/Augoustinos 1995). Neuere Studien sehen in der Frage der Stabilität oder Wandelbarkeit krimineller Verhaltensdispositionen eine mindestens ebenso folgenreiche Dimension der Alltagserklärungen von Kriminalität (Maruna/King 2004): Mit dem Glauben an die Reformierbarkeit des Verhaltens steht und fällt die Unterstützung für Sanktionen, die auf eine Resozialisierung der Täter abzielen, gleichgültig, ob die Ursachen der kriminellen Verhaltensdispositionen eher extern oder intern verortet werden. Zudem hat sich empirisch bestätigt, was schon länger angenommen wurde (Cullen et al. 1985, vgl. Becker 2007): Weniger ein striktes „Entweder-oder“, sondern vielmehr ein „Sowohl-als-auch“ ist typisch für die Alltagserklärungen von Kriminalität. Menschen glauben eher an breit gefächerte Ursachenbündel denn an monokausale Erklärungen. In der englischen Studie von Maruna/King (2004) korreliert die Bevorzugung individueller oder gesellschaftlicher Ursachen von Kriminalität kaum mit der Überzeugung der Resozialisierbarkeit von Straftätern. In ähnlicher Weise zeigen auch Mascini/Houtman (2006; vgl. Klimke 2008: 87), dass die Strafziele von Repression und Resozialisierung nicht als Gegensatz, sondern als Ergänzung wahrgenommen werden.

Dessen ungeachtet ist es in idealtypischer Absicht möglich, einige wenige, grundlegende Deutungsmuster von Kriminalität zu identifizieren, die etwa seit der europäischen Aufklärung im 18. Jahrhundert die Problemwahrnehmungen dominiert haben und die teils nebeneinander existieren, teils sich in zyklischen Konjunkturen überlagern oder abwechseln. Denn der kulturelle „Vorrat“ an unterschiedlichen Deutungsmustern von Kriminalität ist begrenzt (vgl. Groenemeyer 2001).

Das dominante Deutungsmuster sowohl im populären als auch im wissenschaftlichen Diskurs sieht Kriminalität zu einem erheblichen Anteil durch externe Faktoren beeinflusst und sucht in den Biographien und sozialen Lebenslagen der Täter nach (Sozialisations-) Defiziten und Deprivationen, welche eine Motivation zum kriminellen Handeln erklären können. Weit oben in dieser Perspektive rangieren erzieherisches Versagen der Eltern und zerbrochene Elternhäuser, Aufwachsen in ungünstigen sozialen Milieus und Erfahrungen von Armut oder Arbeitslosigkeit. Die Wahrnehmung von Kriminalität wird mithin auf ein Problem sozialer Unterschichten und Randgruppen - heute vor allem ethnischer Minderheiten - sowie auf die symbolisch aufgeladene Jugendkrimina- 
lität verengt und damit die Kri- minalität der Angepassten und Etablierten tendenziell ausgeblendet.

In der kausalen Verknüpfung von Kriminalität mit sozialen Mängellagen unterscheiden sich die Moralstatistiker des frühen 19. Jahrhunderts kaum von den Befürwortern einer wohlfahrtsstaatlichen Kriminalpolitik in der Mitte des 20. Jahrhunderts und offenbar auch nicht von vielen heutigen Menschen (Klimke 2008: 87). Allerdings bestehen erhebliche Unterschiede im Ausmaß des daraus resultierenden Verständnisses gegenüber den Normbrechern und den kriminalpolitischen Konsequenzen. In den europäischen Gesellschaften des 19. Jahrhunderts, die von Industrieller Revolution und starken sozialen Spannungen geprägt waren, wurde Kriminalität primär als ein Problem der ,gefährlichen Klassen“ gesehen, die von Mittel- und Oberschichten als Fremde im eigenen Land und kaum integrierbar in die bürgerliche Gesellschaft wahrgenommen wurden (Becker/Wetzell 2006; Leps 1992; Stedman Jones 1976; Wiener 1990). Die gesellschaftlichen Wahrnehmungen und ebenso die sich entwickelnden Humanwissenschaften dieser Epoche grenzten Kriminalität und andere Formen der Abweichung besonders stark gegen die Erwartungen normgerechten und disziplinierten Verhaltens ab. Damit korrespondierte eine punitive und sozial ausschließende Kriminalpolitik bis hin zur Praxis der Deportationen in Sträflingskolonien (Braithwaite 2004).

Später, in Großbritannien schon seit dem Ende des 19. Jahrhunderts (Wiener 1990: 360), in Deutschland endgültig erst nach dem Ende des Nationalsozialismus, machte diese Haltung einer gelasseneren und verständnisvolleren Interpretation von Kriminalität Platz, in deren Zentrum die Idee der sozialen Benachteilungen stand, für welche die Täter nicht verantwortlich zu machen waren. Dieses Deutungsmuster führte zu einem ,freundlichen“ und optimistischen, auf soziale Inklusion und Resozialisierung ausgerichteten Umgang mit Straftätern, den David Garland (1985) als „,wohlfahrtsstaatliche Strafpolitik“ (penal welfarism) charakterisiert hat. Seine Blütezeit erlebte dieses Deutungsmuster der gesellschaftlichen (Mit-)Verantwortung für Kriminalität in den 1960er und 1970er Jahren, bevor zumindest in den USA und Großbritannien das Pendel wieder spürbar zurückschwang.

In ihrer radikalen, gesellschaftskritischen Variante führte das Deutungsmuster der sozialen Benachteiligungen schließlich zu einer Umkehrung der Rollenverteilung: Die Täter wurden zu Opfern und die Gesellschaft zum eigentlichen Täter erklärt, während die Kriminalitätsopfer weitgehend unbeachtet blieben (Garland 2001: 179). Der kriminalsoziologische Definitions- oder Etikettierungsansatz schlug sich auf die Seite der „underdogs“ (Becker 1967), normalisierte abweichendes Verhalten und deutete Kriminalität stattdessen als Resultat sozial selektiver staatlicher Kontrollhandlungen (Melossi 2001). Auch wenn dieses Deutungsmuster in seiner Reinform über akademische Zirkel hinaus kaum Verbreitung fand, regte es doch wichtige Reformen insbesondere der Jugendkriminalpolitik an, die mit den Begriffen Diversion und Non-Intervention verbunden sind (Albrecht/Ludwig-Mayerhofer 1995), delegitimierte jedoch gleichzeitig mit dem pauschalen Vorwurf des „nothing works" auch wohlfahrtsstaatliche Interventionen (Garland 2001: 67).

Interne Attributionen, die die Ursachen von Kriminalität überwiegend bei den Tätern selbst sehen, sind gegenüber den externen, im weitesten Sinne gesellschaftlichen Zuschreibungen weitaus weniger populär, obwohl sie in der Variante des ,,vernünftigen Verbrechers“ (Cornish/Clarke 1986) die Geschäftsgrundlage unseres Strafrechtssystems bilden. 
Dieses geht trotz der Anerkennung äußerer Beeinflussungen von der individuellen Verantwortlichkeit der mit einem freien Willen und Rationalität ausgestatteten Täter aus. Kriminalität als zweckrationales Handeln zu interpretieren und den Tätern keine sozialen oder psychischen Defizite zu unterstellen, geht auf die klassische Strafphilosophie der Aufklärung, auf Beccaria und Bentham, zurück und findet seit einigen Jahren mit dem Aufschwung von entscheidungstheoretischen Ansätzen in den Sozialwissenschaften eine neue Beachtung (Eifler 2002, 2009; Karstedt/Greve 1996; Sessar 1997). In der Öffentlichkeit wird diese Umorientierung - abgesehen von dem stets vorhandenen Glauben an die Abschreckungswirkung der Strafen - nur indirekt wahrgenommen, wenn es um die kriminalpolitischen Konsequenzen geht: Situationsbezogene Maßnahmen der Kriminalitätskontrolle wie VideoÜberwachung, Einsatz der Polizei oder technische Sicherungen basieren auf einem moralisch neutralen und pragmatischen Rational Choice-Modell menschlichen Handelns und füllen die Lücken einer in die Krise geratenen wohlfahrtsstaatlichen Kriminalpolitik (Garland 2001: 182).

Auch die zweite Variante der internen Ursachenzuschreibung spielt eher im wissenschaftlichen Diskurs als in der Öffentlichkeit eine wieder zunehmende, jedoch sehr kontroverse Rolle. Die im späten 19. und frühen 20. Jahrhundert weit verbreitete Vorstellung, dass zumindest ein relevanter Teil der Kriminalität durch eine Tätergruppe mit tief greifenden Persönlichkeitsstörungen verursacht würde, die auf somatisch verortbare Veranlagungen zurückzuführen seien, war spätestens nach 1945 durch die Ideologie und Verbrechen des Nationalsozialismus nachhaltig diskreditiert (Wetzell 2000). Im Gegensatz zu den pseudowissenschaftlichen Annahmen Cesare Lombrosos und seiner Anhänger über den ,geborenen Verbrecher,, öffnen die neuen Erkenntnisse der Neurowissenschaften und genetischen Forschung bislang verschlossene Fenster auf physiologische Prozesse, die naturwissenschaftlich messbare Entsprechungen menschlicher Wahrnehmungen, Emotionen und Handlungsentscheidungen bilden (siehe Abschnitt 5.2). Während diese Forschungen und insbesondere ihre populärwissenschaftliche Darstellungen in den Medien von den ,traditionellen“ Sozialwissenschaften als eine Herausforderung erkannt werden (Kreissl/Steinert 2008), scheinen sie in den alltagsweltlichen Deutungsmustern von Kriminalität dennnoch keinen massiven Trend von externen zu internen Ursachenzuschreibungen bewirkt zu haben. Der Glaube an angeborene Dispositionen ist für seltene Tätertypen wie z. B. Sexualstraftäter reserviert. Dabei kommt es weniger auf eine Ursachenzuschreibung als auf die Überzeugung an, dass diese Täter nicht besserungsfähig sind, und die Aufgabe der Kriminalpolitik darin gesehen wird, sie unschädlich zu machen (Egg 2000).

\section{Die Rolle von Medien und Politik}

Über die wichtige Rolle der Massenmedien bei der Konstruktion der gesellschaftlichen Problemwahrnehmungen von Kriminalität besteht kein Zweifel. Über Kriminalität erfahren die meisten Menschen durch Medienberichte, nicht aus persönlicher Erfahrung oder Kommunikation, und auch der öffentliche Diskurs über Kriminalität spielt sich in erster Linie in den Medien ab. Darin unterscheidet sich das Thema Kriminalität übrigens nicht von vielen anderen gesellschaftspolitischen Themen wie Klimawandel oder Wirtschaftspolitik. Schwieriger ist es jedoch, die Effekte von Medienberichterstattung auf Individuen, die Wechselwirkungen von Medienangebot, Mediennutzung und Problemwahrnehmungen sowie die Bildung und 
Beeinflussung kollektiver Wahrnehmungen zu untersuchen (Brüchert 2005; Kunczik/Zipfel 2006; Reiner 2007; Reuband 1998; Roberts et al. 2003; Walter 1999).

Die Berichterstattung über reale Kriminalitätsereignisse macht einen wichtigen Teil der Informationsvermittlung in den elektronischen und Printmedien aus und wird durch eine Vielzahl fiktiver Darstellungen von Kriminalität ergänzt. Typische Lokal- und Boulevardzeitungen berichten täglich über ca. 15 bis 20 Kriminalfälle (Baumann 2000: 81; Reuband 2007: 76). Die hoch selektive Auswahl der Fälle nach den Kriterien des Sensationalismus führt dazu, dass Gewaltkriminalität, die nur einen Anteil von ca. 4 Prozent an der registrierten Kriminalität hat, mit 40 Prozent oder mehr der Berichte stark überrepräsentiert ist. Da- rin stimmen die Medien mit der Überschätzung des Anteils von Gewaltdelikten an der Gesamtkriminalität in Bevölkerungsbefragungen überein, ohne dass damit ein kausaler $\mathrm{Zu}$ sammenhang belegt wäre. Es ist nicht überraschend, dass die selektive Medienberichterstattung den in der Bevölkerung gängigen Schwereeinschätzungen von Kriminalität folgt und Mord und Totschlag an die erste Stelle setzt. Am weitaus höchsten ist der Nachrichtenwert von Sexualmorden an Kindern, über die stets ausführlich berichtet wird und die wie kaum ein anderes Verbrechen öffentliche Empörung auslösen (Reiner 2007: 309). Einzelne spektakuläre Kriminalfälle wie der Schul-Amoklauf von Erfurt (2002) mit siebzehn Toten oder der so genannte Ehrenmord an Hatun Sürücü in Berlin (2005) lösen eine wahre Flut von Medienberichten aus und können gesellschaftspolitische Prozesse bis hin zu Gesetzesänderungen in Gang setzen, während die quantitativ viel bedeutsamere Zahl der übrigen Fälle weitaus weniger Beachtung findet.

Qualitativ unterscheidet sich die Berichterstattung nach der Seriosität der Medien: Boulevardzeitungen und Privatsender berichten plakativer und emotionaler über Kriminalität, porträtieren Kriminalität eher als individuelle moralische Verfehlung, verzichten eher auf Ambiguitäten und bedienen die punitiven Instinkte ihrer Rezipienten und Rezipientinnen damit mehr als Qualitätszeitungen und das öffentlich-rechtliche Fernsehen (Roberts et al. 2003: 78). Allerdings sind Angleichungstendenzen dieser Mediengattungen unter den Wettbewerbsbedingungen des Marktes unverkennbar (Pfeiffer et al. 2004; Reuband 2007). Als exemplarisch hierfür kann ein Titelbild des Nachrichtenmagazins „Der Spiegel“ (15/1998) inmitten einer Welle öffentlicher Beunruhigung über Jugendkriminalität gelten, das dieses Thema mit einer dramatischen Bildcollage und der Überschrift „Die kleinen Monster. Warum immer mehr Kinder kriminell werden“ einleitete.

In einer aktuellen Studie zu den Zusammenhängen von Medienkonsum und Kriminalitätseinstellungen fanden Pfeiffer et al. (2004, 2005; vgl. Dowler 2003) einen signifikanten Effekt der Nutzung von Privatfernsehen auf eine dramatisierende Problemwahrnehmung von Kriminalität, der allerdings weitaus schwächer war als andere Variablen wie z. B. das Bildungsniveau; die dramatisierende Problemwahrnehmung wiederum ging mit einem stärkeren Strafbedürfnis einher. Korrelative Zusammenhänge wie diese sagen allerdings wegen des massiven Einflusses der Selbstselektion von Mediennutzern wenig über kausale Effekte aus (Reiner 2007: 322). Menschen wählen Medien nach ihren individuellen Präferenzen relativ frei aus, können jedoch in ihren Wahrnehmungen und Einstellungen dann auch beeinflusst, beispielsweise weiter bestärkt werden. Ebenso sind gesellschaftliche Entwicklungstrends der öffentlichen Meinung zu kriminalitätsbezogenen Themen selbstverständlich nicht ohne die Mitwirkung der Medien denkbar, ohne dass Ver- 
schwörungstheorien einer einseitigen Manipulation der Menschen durch Medien gerechtfertigt sind (Garland 2001: 146). Zur Verstärkung populistischer Problemwahrnehmungen und Deutungsmuster kann beitragen, dass die überwiegend privaten Medienanbieter ihren wirtschaftlichen Interessen folgend vor allem das berichten, wonach eine hohe Nachfrage besteht, und dies in einer Auflage- und Reichweitenfreundlichen Weise tun. Aber auch für die Veränderung und Schärfung kollektiver Problemwahrnehmungen wie z. B. von Umweltkriminalität oder fa- milialer Gewalt gegen Kinder werden die Medien von Interessengruppen und Moralunter- nehmern als ein unverzichtbares Vehikel benutzt (Groenemeyer 2003; Schetsche 2008).

Schließlich spielen auch das politische System, politische Parteien und individuelle Politiker eine Rolle bei der Konstruktion der Problemwahrnehmungen von Kriminalität, wiederum vermittelt über die Massenmedien. Scheerer (1978) hat in diesem Zusammenhang von einem ,politisch-publizistischen Verstärkerkreislauf“ gesprochen, allerdings in einem etwas anderen Sinne als es in den neueren Zeiten des „punitiven Populismus“ verstanden wird. Beschrieb Scheerer die erfolgreichen Anstrengungen von Regierung und staatlichen Behörden, gegen den Trend der öffentlichen Meinung eine Liberalisierung der Drogengesetze zu verhindern und diesen Meinungstrend umzukehren, so geht es heute um die Neigung von Politikern und Parteien, im demokratischen Konkurrenzkampf mit „Law and Order"-Forderungen aus den Unsicherheitsgefühlen und Strafbedürfnissen der Bevölkerung politisches Kapital zu schlagen und Wählerstimmen zu gewinnen (Pratt 2007; Roberts et al. 2003; Shea 2009). Insbesondere konservative und rechtsgerichtete Parteien, und am konsequentesten in den USA, haben diese Strategie häufig angewendet.

Ein inzwischen klassisches Beispiel ist der amerikanische Präsidentschaftswahlkampf 1988, in dem eine konkrete Gewalttat eines verurteilten Mörders während seines Hafturlaubs in zwei TV-Werbesendungen sehr erfolgreich verwendet wurde, um den Kandidaten der Demokratischen Partei, Michael Dukakis, zu diskreditieren und die Wählerstimmung zugunsten des republikanischen Kandidaten George $\mathrm{H}$. Bush herumzudrehen (Beckett 1997; Newburn/Jones 2005). Diese Strategie ist deswegen populistisch, weil es in vielen Fällen einfacher und erfolgversprechender ist, die expressiven, emotional aufgeladenen Reaktionen von Furcht und Vergeltung in der Bevölkerung zu aktivieren, die die Basis einer punitiven Kriminalpolitik bilden, als das Verständnis für eine rationale, nutzenorientierte Kriminalpolitik auf der Basis wissenschaftlicher Erkenntnisse zu wecken (H.-J. Albrecht 2004: 497). Wenn dieser populistische Mechanismus funktioniert, kann er im Sinne des „politisch-publizistischen Verstärkerkreislaufs“ (Scheerer) die öffentliche Meinung weiter in die Richtung dramatisierender und vereinfachender Problemwahrnehmungen verstärken. Sich einem solchen Meinungstrend entgegen zu stellen, fällt auf Mehrheiten angewiesenen Politikern und Politikerinnen verständlicherweise schwer. US-Präsident Barack Obama, in der Vergangenheit eher ein Gegner der Todesstrafe, stellte sich im Präsidentschaftswahlkampf 2008 noch am selben Tage gegen die Entscheidung des Obersten Gerichtshofes, die Anwendung der Todesstrafe bei Vergewaltigung von Kindern für verfassungswidrig zu erklären (New York Times, 25.6.2008). Jedoch sprechen die Erfahrungen in Deutschland eher gegen den Erfolg dieser populistischen Strategie (siehe Abschnitt 4.5). 
Der symbolische Einsatz des Themas Kriminalität in der politischen Rhetorik ist ein Aspekt, die faktische Veränderung der Praxis staatlicher Sozialkontrolle durch Umsetzung kriminalpolitischer Programme ist ein weiterer Aspekt des Verhältnisses zwischen öffentlichen Problemwahrnehmungen und staatlicher Politik. Auch hier sind Wechselwirkungen im Sinne einer gegenseitigen Verstärkung denkbar, die aber nicht ausschließlich in die Richtung dramatisierender Problemwahrnehmungen und punitiver Reaktionen gehen müssen. Das Beispiel für einen entgegengesetzten Trend ist die Abschaffung der Todesstrafe in Deutschland im Jahr 1949. Zu diesem Zeitpunkt zeigten Meinungsumfragen noch eine deutliche Mehrheit für die Todesstrafe an, und erst nach ihrer Abschaffung wandelte sich die öffentliche Meinung zu einer mehrheitlich ablehnenden (Hood 2001; Kury/Kania/ Obergfell-Fuchs 2004; Zimring 2004: 10). Inwieweit die Beobachtung, dass in diesem Fall die öffentliche Meinung der Politik nachgefolgt ist, auch einen kausalen Gehalt hat, lässt sich allerdings schwer belegen.

\subsection{Die Entwicklung der Problemwahrnehmungen und Strafmentalitäten: Eine neue punitive Kultur?}

Problemwahrnehmungen, Deutungsmuster und Strafbedürfnisse bilden zusammen einen kriminalitätsbezogenen Einstellungskomplex, der die staatliche Kriminalpolitik mit beeinflusst und generell eine wichtige Rolle dabei spielt, wie eine Gesellschaft mit Kriminalität und Straftätern umgeht. Diese Strafmentalitäten unterliegen, wie schon mehrfach angeklungen ist, Wandlungsprozessen, deren Verläufe und Ursachen von den Sozialwissenschaften noch nicht sehr gut verstanden werden. Seit einer Reihe von Jahren zeichnen sich Veränderungen ab, die unter dem Schlagwort der „punitiven Wende“ diskutiert werden (Crawford 2002; Lautmann/Klimke/Sack 2004; Pratt et al. 2005). Die umfassendste und komplexeste Beschreibung und Einordnung dieser Entwicklung am Beispiel der USA und Großbritanniens stammt von David Garland (2001, 2004, 2007). Inwiefern diese Entwicklungen auch auf Deutschland und andere europäische Länder zutreffen, ist eine gegenwärtig kontrovers diskutierte Frage, die einen sehr guten Einstieg in die Untersuchung zeitlicher Veränderungen und internationaler Vergleiche von Problemwahrnehmungen und Strafmentalitäten bietet (H.-J. Albrecht 2004; Hess/Ostermeier/Paul 2007; Klimke 2008; Kury/Ferdinand 2008; Lautmann/Klimke/Sack 2004; Tonry 2007). Garlands Thesen sollen daher im Folgenden sehr knapp referiert werden.

Garland stellt zwei Typen des gesellschaftliche Umgangs mit Kriminalität gegenüber: die „wohlfahrtsstaatliche Strafpolitik“ (penal welfarism) mit der Blütezeit in den 1960er und 1970er Jahren und die „Kontrollkultur“ (culture of control), die sich seit den 1980er Jahren entwickelt hat. Idealtypisch war die wohlfahrtsstaatliche Strafpolitik durch gelassene und wenig besorgte Problemwahrnehmungen gekennzeichnet; die Kriminalpolitik verfolgte resozialisierende Ziele und wurde weitgehend Experten und Expertinnen überlassen, von denen man rationale und erfolgreiche Konzepte der Behandlung von Straftätern erwartete. Unter dem Einfluss des starken Anstiegs der Kriminalitätsraten bei gleichzeitiger Abkehr vom Ideal des großzügigen Wohlfahrtsstaates erlangte das Thema Kriminalität in den USA und Großbritannien dann eine neue und wesentlich prominentere Wertigkeit (Salienz), die es trotz des inzwischen deutlichen quantitativen Rückgangs der Kriminalität bis heute behalten hat. 
Kriminalitätsgefahren werden in der „High Crime Society“ überzogen dramatisiert, von Politikern und Politikerinnen gezielt für Wahlkampfzwecke ausgenutzt (Beckett 1997) und bestimmen weite Politikfelder über die Kriminalpolitik hinaus (Simon 2007). Das Ideal der Resozialisierung der Täter ist diskreditiert, Sicherheit und Sicherheitsgefühle stehen im Mittelpunkt dieser Kultur (Albrecht 2007; Loader/Walker 2007). Damit geht eine Perspektivverschiebung vom Täter zum Opfer einher (Hassemer/Reemsta 2002; Walklate 2007). Täter können nicht mehr mit Verständnis und dem Bemühen um Resozialisierung rechnen, sondern sollen bestraft und wenn nötig durch lange Gefängnisstrafen unschädlich gemacht werden. Harte Strafen bis hin zur Todesstrafe erfüllen primär symbolische Bedürfnisse der Bekräftigung moralischer Normen (acting out), können jedoch Kriminalität kaum verhindern, während andererseits Sicherheit durch Kontrollmaßnahmen, z. B. durch technische Überwachung, hergestellt werden soll, die nicht auf den Täter (und die Ursachen seiner Straffälligkeit) abzielen, sondern auf die Kontrolle von Situationen und Tatgelegenheiten. Programmatisch spiegelt sich diese Zweiteilung der Zielrichtungen von Kriminalpolitik in dem Nebeneinander zweier kriminologischer Denkansätze wider: Einer „Kriminologie des Alltags“ (z. B. Routine Activities Approach) einerseits und einer „Kriminologie des Anderen" andererseits, die ähnlich wie im 19. Jahrhundert die Distanz zwischen dem Normalen und dem Abweichenden stark betont und Straftäter tendenziell pathologisiert.

Garlands Diagnose wird durch eine Fülle empirischer Evidenzen aus den USA und Großbritannien belegt, die von Befragungsergebnissen zu Kriminalitätsfurcht und Strafeinstellungen über die Rolle des Themas Kriminalität im politischen Meinungskampf bis zum explosionsartigen Anstieg der Gefangenenzahlen in den USA oder dem massiven Ausbau der Video-Überwachung in Großbritannien reichen. Weniger klar zu beantworten ist die Frage, inwiefern Deutschland und andere europäische Länder dem angelsächsischen Typus der ,high crime societies“ entsprechen oder sich zumindest auf dem Weg dorthin befinden (Sack 2007, 2008). Ich möchte dieser Frage hier wiederum vorrangig in Hinblick auf die gesellschaftlichen Wahrnehmungen des sozialen Problems Kriminalität und nicht in Hinblick auf die Veränderung der staatlichen Sozialkontrolle nachgehen.

International vergleichende Befragungen ergeben eine große Spannbreite in den kriminalitätsbezogenen Einstellungen zwischen den europäischen Ländern. Großbritannien, Irland sowie viele ost- und südeuropäische Länder zeigen nicht nur hohe Werte der Kriminalitätsfurcht, sondern auch ein größeres Strafverlangen als Skandinavien, Frankreich, Deutschland und andere west- und mitteleuropäische Länder. Bei der standardisierten Frage nach der bevorzugten Strafe für einen Wiederholungstäter, der wegen eines Wohnungseinbruchs vor Gericht steht, halten 51 Prozent der englischen, aber nur 12 Prozent der schweizer, 13 Prozent der französischen und 19 Prozent der deutschen Befragten eine Gefängnisstrafe für angemessen (van Dijk/van Kesteren/Smit 2007: 149; vgl. Oberwittler/Höfer 2005); zudem ist das Strafverlangen in England zwischen 1989 und 2004 sehr viel stärker angestiegen als in den Vergleichsländern.

Ein Kristallisationspunkt für die punitive Wende in Großbritannien war der bis heute im kollektiven Gedächtnis präsente Fall des zweijährigen James Bulger, der 1993 von zwei zehnjährigen Jungen grausam umgebracht wurde. Anhand dieses Einzelfalles und eines sehr ähnlichen Falles, der sich 1994 in Norwegen ereignete, hat David Green (2007, 2008) die großen Unterschiede der gesellschaftlichen Reaktionen auf Kriminalität zwischen der 
„high-crime society“ Großbritannien und einem Land herausgearbeitet, das sich eher am entgegengesetzten Pol der Strafkulturen befindet. In Großbritannien reagierte die Öffent- lichkeit mit heftigen Verurteilungen und Dämonisierungen der Jungen als eine Art Monster.

$\mathrm{Zu}$ der dauerhaft aufgeheizten öffentlichen Meinung trugen eine sensationalistische Berichterstattung der Medien und populistische Äußerungen vieler Politiker bei. In der öffentlichen Thematisierung wurde der Fall als die Spitze eines Eisbergs von Jugendgewalt und als Zeichen des moralischen Niedergangs der Gesellschaft interpretiert. Green hebt insbesondere zwei Elemente in diesem Diskurs hervor, die die Abkehr vom früheren ,penal welfarism“ deutlich machen: Die Experten und Expertinnen (Psychologen, Pädagogen etc.) wurden nicht nur nicht gehört, sondern als zu verständnisvoll verächtlich gemacht, und Politiker und Politikerinnen sowohl der Regierung als auch der Opposition reagierten mit populistischen Rufen nach härteren Strafen gegen Kinder und Jugendliche (die dann auch gesetzlich realisiert wurden) und trugen damit zur Diskreditierung der bestehenden Behandlungsformen von delinquenten Kindern bei.

Im Kontrast dazu rief der Fall des ebenfalls grausamen Todes eines fünfjährigen Mädchens durch die Hände dreier sechsjähriger Jungen auf einem Spielplatz in Trondheim kei- ne öffentliche Empörung und keine Forderungen nach harten Strafen hervor. Die Medien berichteten nur sehr zurückhaltend und ließen vorrangig Experten und Expertinnen zu Wort kommen. Die in den Medien favorisierte Interpretation, der sich auch die Eltern des Opfers anschlossen, sah sowohl das Mädchen als auch die drei Täter als „Opfer eines tragischen Vorfalls“. Die Täter wurden nicht aus ihren Familien herausgenommen, sondern therapeutisch betreut und besuchten weiterhin einen Kindergarten.

Green hebt bei der Erklärung dieser eklatanten Unterschiede insbesondere die Rolle des in Großbritannien härteren Wettbewerbs sowohl der Medien um Leser und Leserinnen als auch der Politiker und Politikerinnen um Wählerstimmen hervor. Eine dramatisierende und populistische Thematisierung von Kriminalität sei demnach in (anglo-amerikanischen) Mehrheitsdemokratien wahrscheinlicher als in (kontinentaleuropäischen) Konsens-Demokratien. Auch wenn die Strafmentalitäten sicher nicht hinreichend mit Merkmalen des Parteien- und Regierungssystems erklärt werden können, spricht Green dennoch über die bei- den Länder hinausgehende bedeutsame Unterschiede innerhalb der westlichen, demokrati- schen Gesellschaften an, die erklären können, warum die Voraussetzungen für eine Über- nahme des Strafpopulismus US-amerikanischer Prägung in Deutschland und anderen euro- päischen Ländern deutlich ungünstiger sind (Lacey 2008; Karstedt 2007b; Pratt 2007; Tonry 2004, 2007).

In vielen europäischen, korporatistisch geprägten Ländern liegt die Gestaltung der Kriminalpolitik in der Hand von bürokratischen Eliten und Experten bzw. Expertinnen, die durch ihren Status als Karriere-Beamte stärker vor dem Einfluss eines latent vorhandenen Strafpopulismus geschützt sind als beispielsweise vom Volk gewählte Richter und Staatsanwälte in den USA. Dadurch wird der ,,politisch-publizistische Verstärkerkreislauf" wenn nicht völlig unterbrochen, so doch erheblich gebremst (Jacobs/Kleban 2003; Roberts et al. 2003: 74). Andere Autoren und Autorinnen weisen auf teils weit zurückreichende gesellschafts- und mentalitätsgeschichtliche Unterschiede (Savelsberg 2004; Whitman 2003, 2005) und auf die bereits mehrfach erwähnte Rolle wohlfahrtsstaatlicher Arrangements hin, 
die vor existenziellen Unsicherheiten schützen (Cavadino/Dignan 2006; Hummelsheim et al. 2010; Karstedt 2007a).

Eine systematische empirische Analyse der Entwicklung der Problemwahrnehmungen und Strafeinstellungen in Deutschland steht noch aus bzw. fällt aufgrund lückenhafter Datengrundlagen schwer (Kury/Brandenstein/Obergfell-Fuchs 2009; Lautmann/Klimke 2004). Wenn z. B. eine 1976 durchgeführte Befragung 81 Prozent Zustimmung zu der Aussage „Es sollten längere und härtere Strafen verhängt werden“ erbrachte (Murck 1978: 207), wirft dies ein Schlaglicht auf die Existenz eines breiten punitiven Potenzials schon lange vor Beginn der Spätmoderne. Eine von 1970 bis 2003 reichende Befragungsreihe ergab eine moderate Zunahme des Strafverlangens bei Diebstahl (Reuband 2007: 100): Der Anteil der Befragten, die eine Haftstrafe ohne Bewährung für angemessen halten, stieg von 4 auf 11 Prozent, eine Bewährungsstrafe befürworteten 197012 und 200320 Prozent. Die dramatisch gewachsene Befürwortung von Gefängnisstrafen für prügelnde Ehemänner (1970: 2 \%, 2003: $40 \%$ ) reflektiert die gesellschaftliche Neubewertung häuslicher Gewalt; Haschischkonsum auf der anderen Seite ruft 2003 ein geringeres Strafbedürfnis hervor als 1970.

Ein anderer Indikator punitiver Überzeugungen, für den relativ lange Zeitreihen vorliegen, ist die Einstellung zur Todesstrafe. In der westdeutschen Bevölkerung insgesamt ist die Zustimmung zur Todesstrafe von einer knappen Mehrheit in der frühen Nachkriegszeit auf 22 Prozent im Jahr 2005 gesunken - unterbrochen von zwei gegenläufigen Trends in den 1970er und frühen 1990er Jahren (Kury/Kania/Obergfell-Fuchs 2004: 65; Institut für Demoskopie Allensbach, pers. Mitteilung). Unter Jura-Studierenden im ersten Semester jedoch wuchs die Befürwortung der Todesstrafe von 11,5 im Jahr 1977 auf 32 Prozent im Jahr 2007 an (Streng 2008: 768). Während 197735 Prozent der Studierenden für eine Abschaffung der lebenslangen Freiheitsstrafe plädierten, wollten dies 2007 nur noch 2 Prozent; 33 Prozent vertraten nun die Auffassung, diese Strafe sei ,für manche Straftaten noch eine zu milde Strafe“ (1977: $7 \%$ ). Hier zeichnet sich eine deutliche Hinwendung zu punitiven Einstellungen ab, die vor allem deswegen bedenklich stimmt, weil Jura-Studierende den Nachwuchs für eben die bürokratischen Eliten darstellen, die in den Diskussionen über die Entwicklung der Kriminalpolitik als wichtiges Bollwerk gegen eine punitive Wende gelten.

Eine deutliche Zuspitzung der medial vermittelten Problemwahrnehmungen, die streckenweise Züge einer moralischen Panik aufwiesen, zeigte sich in Deutschland bei Sexualstraftaten. Während sich die Zahl der Sexualmorde an Kindern langfristig rückläufig entwickelt hat, wurden einzelne Fälle in den 1990er Jahren wesentlich ausführlicher thematisiert als zuvor. Das Sexualstrafrecht erlebte eine Reihe von gesetzlichen Verschärfungen (Frommel 2005; Klimke/Lautmann 2006), und die Entwicklung der Strafpraxis deutet darauf hin, dass den gestiegenen Problemwahrnehmungen und gesetzlichen Änderungen auch ein Trend zu längeren Freiheitsstrafen folgte (KuryKania/Obergfell-Fuchs 2004; Weigend 2001).

Die populistische Ausbeutung des Themas Kriminalität in politischen Wahlkämpfen hatte in Deutschland in der jüngeren Vergangenheit bislang einmal Erfolg: In der Hamburger Bürgerschaftswahl von 2001 erhielt die so genannte Schill-Partei mit einem ausschließlich auf „Law and Order“ basierenden Wahlprogramm knapp 20 Prozent der Stimmen 
(Reuband 2002). Der Versuch des CDU-Kandidaten Roland Koch während des hessischen Landtagswahlkampfs 2008, eine in den Medien viel berichtete Gewaltstraftat zweier nicht-deutscher Jugendlicher zu nutzen, um seine Popularität mit Forderungen nach einer radika- len Verschärfung des Jugendstrafrechts zu steigern, scheiterte jedoch. Die öffentliche Mei- nung folgte in diesem Fall nicht dem populistischen Politiker, sondern der besonnenen $\mathrm{Ge}$ - genstimme der mehr als eintausend Fachleute, die das bestehende Jugendstrafrecht in einer öffentlichen Stellungnahme verteidigten (Heinz 2008b).

Dieses aktuelle Beispiel zeigt die Grenzen eines punitiven Populismus in Deutschland auf. Daniela Klimke (2008: 86 ff.) zeigt auf der Basis quantitativer und qualitativer Interviews zu kriminalitätsbezogenen Einstellungen, dass klassische wohlfahrtsstaatliche Auffassungen über die sozialen Ursachen von Kriminalität ebenso lebendig sind wie der Glau- be an die Wirksamkeit sozialpolitischer und kriminalpräventiver Maßnahmen und eine Skepsis gegenüber harten Strafen. Melanie Becker (2007) fand in qualitativen Interviews eine stärkere Verbreitung moralischer Deutungsmuster von Kriminalität, verbunden mit der Befürwortung punitiver Reaktionen auf Kriminalität, die jedoch für viele Befragte mit der Befürwortung sozialpräventiver Instrumente vereinbar waren (vgl. Mascini/Houtman 2006).

Die meisten verfügbaren Zeitreihen zeigen sowohl für die Kriminalitätsfurcht als auch die Wahrnehmung von Kriminalität als gesellschaftliches Problem einen fallenden Trend seit dem Anfang der 1990er Jahre, als es im Anschluss an Maueröffnung und Wiedervereinigung zu einer Verschärfung der Problemwahrnehmungen gekommen war (Dittmann 2005, 2009; Reuband 1996, 2007). Das Niveau der Kriminalitätsfurcht ist sogar auf den niedrigsten Wert seit ihrer regelmäßigen Messung in den frühen 1980er Jahren gefallen. Bei einer der ersten deutschen Opferbefragungen 1973 in Stuttgart antworteten 48 Prozent der Befragten auf die Standardfrage zur Kriminalitätsfurcht, dass sie sich unsicher fühlten, davon 18 Prozent sogar ,sehr unsicher“ (Stephan 1976: 87), 2006 fühlten sich in deutschen Großstädten (unter Verwendung derselben Frage) nur noch 26 Prozent unsicher (Statistisches Bundesamt et al. 2008: 306).

Bei regelmäßigen Bürgerbefragungen in Frankfurt/M. sank die Nennung von Kriminalität bei der offenen Frage (ohne Antwortvorgabe) nach den größten Problemen der Stadt von 57 im Jahr 1993 auf 13 Prozent im Jahr 2007 und liegt nun immer noch auf dem dritten Platz (Stadt Frankfurt 2008). Der stabile Rückgang der Bedrohungsängste seit 1993 ist in den jährlich durchgeführten Befragungen der $\mathrm{R}+\mathrm{V}$ Versicherung $\mathrm{zu}$ erkennen, die auch die seit 2001 sprunghaft gestiegenen Ängste vor Terroranschlägen anzeigen (Abbildung 10).

Der rückläufige Trend und das im internationalen Vergleich relativ niedrige Niveau bedeuten allerdings nicht, dass die Bedrohungswahrnehmungen in Deutschland nicht als problematisch interpretiert werden können - sie sind es in dem Maße, in dem sie im Vergleich zu den tatsächlichen Risiken durch Kriminalität überzogen erscheinen und dadurch negative Rückwirkungen auf Bereiche wie Wohlbefinden, Alltagsleben, Zwischenmenschliches und Systemvertrauen etc. haben.

Wenn etwa jeder Vierte Angst vor Kriminalität hat und sich in seinem Wohngebiet unsicher fühlt, deutet einiges auf einen Problemgehalt hin, der jedoch eher nicht mit den überstrapazierten Begriffen „Globalisierung“ und „Neoliberalismus“ in Verbindung gebracht werden sollte, denen eine romantische Verklärung der Vergangenheit anhaftet. 


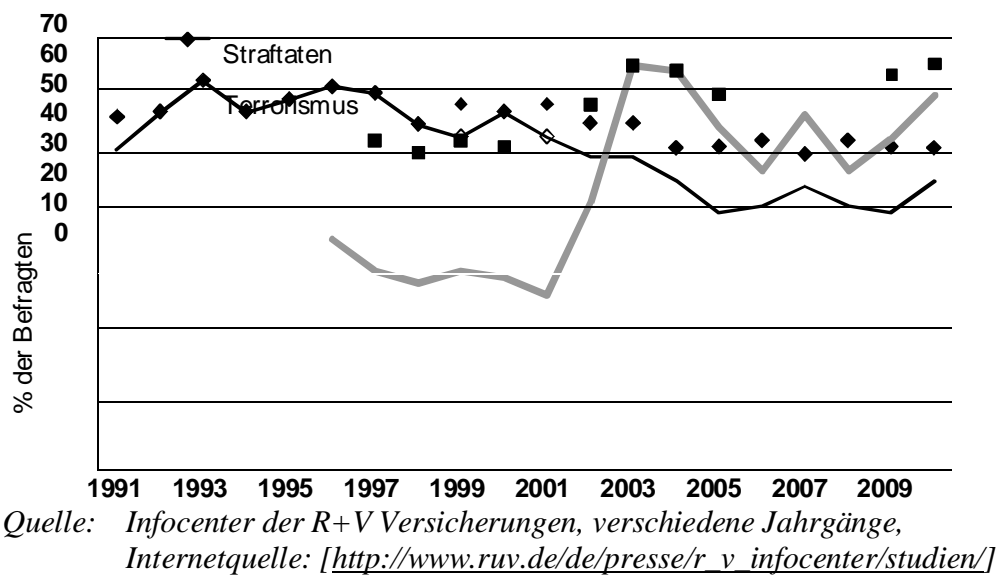

\section{Theorien und Forschungserkenntnisse zur Erklärung von Delinquenz und Kriminalität}

\section{1 Überblick}

Das Bedürfnis, die Ursachen von Kriminalität zu verstehen (und dadurch auch Mittel zu ihrer Vermeidung zu finden), hat seit mehr als einhundert Jahren eine Vielzahl von Theorien verschiedener Wissenschaftsdisziplinen hervorgebracht und damit eine Forschungsliteratur, die Bibliotheken füllt und nur schwer zu überblicken ist. Da das einschlägige Fach „Kriminologie“, zumal in Deutschland, eher ein interdisziplinäres Forschungsfeld als eine eigenständige Wissenschaftsdisziplin ist (Karstedt 2000; Karstedt/Oberwittler 2004), kamen und kommen maßgebliche Impulse für die Erforschung von Kriminalität aus den Bezugsdisziplinen, allen voran Soziologie und Psychologie, aber auch Psychiatrie, Wirtschaftswissenschaften, Geographie und Biologie. Bis in die 1960er Jahre wurde die deutsche kriminologische Forschung stark von der Psychiatrie und Rechtswissenschaft dominiert und hat sich erst verspätet und zögerlich der Soziologie geöffnet, welche in den USA schon seit Beginn des 20. Jahrhunderts die Leitdisziplin gewesen war (Oberwittler/Höfer 2005; Savelsberg/Sampson 2002).

Jede dieser Disziplinen hat unterschiedliche Perspektiven auf das Phänomen Kriminalität und rückt diese in ihren Theorieansätzen verständlicherweise in den Vordergrund. Soziologen interessieren sich vorwiegend für gesellschaftliche Zusammenhänge, Psychologen eher für Fragen der Persönlichkeit, Psychiater sehen Kriminalität als potenziell krankhafte Verhaltensstörung und so weiter. Die Konkurrenz zwischen diesen Perspektiven wird umso größer, je „monokausaler“ ihre Theorien sind und je stärker sie mit dem Anspruch auf Allgemeingültigkeit vertreten werden. Der Wirtschaftswissenschaftler Gary Becker beispiels- 
weise wollte Kriminalität schlicht mit der Erwartung des Täters erklären, dass der Nutzen einer kriminellen Handlung ihre Kosten übersteigt: „Some persons become ,criminals ${ }^{\text {ee }}, \ldots$ not because their basic motivation differs from that of other persons, but because their benefits and costs differ“ (Becker 1968: 176). Eine andere, selbst ernannte „General Theory of Crime“" (Gottfredson/Hirschi 1990) sieht die Wurzel jeder Form von Kriminalität in der geringen Selbstkontrolle der Täter und Täterinnen aufgrund mangelnder Erziehung in den ersten Lebensjahren. Neurobiologische Forschungen schließlich verweisen auf die Rolle von Hormonen und Gehirnaktivitäten bei der Genese krimineller Handlungen (Markowitsch/Siefer 2007; Roth/Lück/Strüber 2006).

Diese disziplinären Ausrichtungen sind zwar legitim und forschungspragmatisch unvermeidlich, werfen jedoch einige grundsätzliche Fragen auf. Auch wenn in diesem Beitrag vorrangig eine soziologische Perspektive verfolgt wird, ist es dennoch hilfreich, über das Spannungsverhältnis der verschiedenen Erklärungsansätze untereinander und zu ihrem Forschungsgegenstand nachzudenken. Mein hier schon vorweggenommener Standpunkt ist, dass integrative Theorieansätze, die verschiedene Einflussfaktoren auf unterschiedlichen (Mikro- und Makro-)Ebenen miteinander verbinden, am besten zur Analyse der Ursachen von Delinquenz und Kriminalität geeignet sind, da keine Disziplin ein Deutungsmonopol beanspruchen kann.

Eine erste Frage richtet sich auf die Vielfalt des Untersuchungsgegenstands Kriminalität: Was verbindet so unterschiedliche Phänomene wie Graffiti-Sprühen und Bankraub, Versicherungsbetrug und sexuellen Missbrauch, und ist eine allgemeine Kriminalitätstheorie angesichts der Diversität der Formen von Delinquenz und Kriminalität (Hess/Scheerer 1997: 84) überhaupt denkbar? Diese Frage ähnelt der sprichwörtlichen Unklarheit, ob das Glas halb voll oder halb leer ist. Einerseits unterscheiden sich verschiedene Kriminalitätsphänomene ohne Zweifel sehr stark (dieses Argument wird unten weiterverfolgt), andererseits haben sie doch definitionsgemäß eine wichtige Gemeinsamkeit: Sie bilden einen strafbaren Normbruch (siehe Abschnitt 2). Da diejenigen, die Delikte begehen, in der Regel wissen, dass sie eine Norm verletzen und dafür sanktioniert werden könnten, ist das allen Kriminalitätsphänomen gemeinsame Explanandum, warum jemand einen strafbaren Normbruch begeht (Wikström 2007a). Auf dieser handlungstheoretischen Ebene machen dann allgemeine Erklärungsansätze wie die, dass Normbrüche durch mangelnde Bindungen an die konventionelle Gesellschaft (Kontrolltheorie), durch Erlernen in sozialen Gruppen (Lerntheorie) oder wegen fehlender Voraussicht der Konsequenzen (Theorie der niedrigen Selbstkontrolle) wahrscheinlicher werden, durchaus Sinn, weil sie einen elementaren Aspekt des abweichenden Verhaltens ansprechen.

Der kriminalsoziologische Definitionsansatz hat gegen diese ätiologische, auf den Täter fokussierte Sichtweise (Ätiologie: Lehre von den Krankheitsursachen) grundlegende Einwände erhoben. Dieses in den 1950er und 1960er Jahren entwickelte Paradigma, dass Kriminalität eine Eigenschaft ist, die Personen und Handlungen von der Gesellschaft (von den Opfern, Beobachtern sowie vor allem den Instanzen der staatlichen Sozialkontrolle) angeheftet wird, hat konsequenterweise die Forderung nach sich gezogen, diese Bewertungsund Stigmatisierungsprozesse in den Mittelpunkt der Forschung zu stellen. „Abweichendes Verhalten [ist] das, was andere als abweichend definieren. Es ist keine Eigenschaft oder ein 
Merkmal, das dem Verhalten als solchem zukommt, sondern das an das jeweilige Verhalten herangetragen wird“" (Sack 1968: 470, vgl. Erikson 1978 [1966]; Peters 2009).

Im Kontext der gesellschaftskritischen Strömung der 1960er Jahre machten die Anhänger des Definitionsansatzes in allererster Linie die Instanzen des Strafrechtssystems für die „Produktion“ der Kriminellen verantwortlich, indem diese durch eine ungerechte Anwendung der Strafnormen aus der breiten Menge all derer, die gegen Normen verstoßen, vorrangig Unterschichtsangehörige und ohnehin Marginalisierte herausfilterten. Nicht zufällig interessierten sich Pioniere des Definitionsansatzes wie Howard S. Becker (1981 [1963]) eher für die Grauzonen delinquenten Verhaltens wie z. B. Homosexualität und Drogenkonsum, in denen die Wandelbarkeit der Definitionen und Spielräume der sozialen Kontrolle ungleich größer waren als im Kernbereich krimineller Handlungen. Diese Auffassung basierte auch auf einer Fehlinterpretation der erst im Entstehen befindlichen Dunkelfeldstudien, die den Eindruck zuließen, dass delinquentes Verhalten in der Gesellschaft mehr oder weniger gleich- oder gar zufällig verteilt sei.

Innerhalb des Definitionsansatzes lassen sich grob zwei unterschiedliche Richtungen unterscheiden. Erstens gibt es einen mit den ätiologischen, täterorientierten Theorien kombinierbaren Ansatz, der den Anteil der Normanwendung und Sanktionierung an der biographischen Entwicklung und Verstärkung krimineller Karrieren (durch Stigmatisierung, selffulfilling prophecy etc.) untersucht. Hierfür stehen unter anderem die Konzepte der sekundären Devianz (Lemert 1967; vgl. Lamnek 2007: 223; Matsueda 2006) und des Reintegrative Shaming (Braithwaite 1989). Zweitens gibt es den ,radikalen Definitionsansatz“, der darauf insistiert, dass eine Erforschung der Kriminalitätsursachen auf der Ebene des Täters und seiner Handlungen unmöglich sei, weil die Definition der kriminellen Handlung erst mit der sozialen Reaktion der Gesellschaft beginne und nicht schon mit der Handlung selbst (Peters 1996; Sack 1969). Diese Verabsolutierung des Grundgedankens des Definitionsansatzes machte aus den Tätern und Täterinnen jedoch passive Opfer und sprach ihnen nicht nur jegliche Verantwortung, sondern auch die Entscheidungsfreiheit für oder gegen Normabweichungen ab. Damit wurde der Ansatz des symbolischen Interaktionismus Herbert Meads, auf den sich der Definitionsansatz beruft, unfreiwillig konterkariert, worauf bereits Lemert (1974: 458) hinwies: „Labeling unfortunately conveys an impression of interaction that is both sociologistic and unilateral ... it leaves little or no place for human choice at either level of interaction." Die Erkenntnis, dass abweichendes Verhalten durch die Existenz von Normen definiert wird und dass diese selektiv angewendet werden, macht daher die Frage, warum Normen gebrochen werden, keinesfalls überflüssig (vgl. Pfeiffer/Scheerer 1979: 58; Hess/Scheerer 1997: 118; Lüdemann/Ohlemacher 2002: 51).

Trotz des verbindenden Elements des Normbruchs gibt es dennoch keine allgemeine und zugleich monokausale Theorie, die alle Erscheinungsformen von Kriminalität gleichermaßen überzeugend erklären kann und wirklich breite Anerkennung gefunden hätte. Gewalt auf deutschen Schulhöfen scheint nach anderen Erklärungen zu verlangen als tödliche Drogenkriege in Kolumbien. Während die Gewaltneigung von Kindern deutlich von Aspekten der Persönlichkeit und der Eltern-Kind-Beziehung beeinflusst wird (z. B. Lösel/ Bliesener 2003), stehen bei den Drogenkriegen eher die Mechanismen illegaler Märkte und die Schwäche des staatlichen Gewaltmonopols im Vordergrund (z. B. Reuter 1983; Wald- 
mann 2007). Ein Kriterium für die Auswahl geeigneter Erklärungsansätze ist demnach ihre empirische Relevanz.

Die entwicklungspsychologischen Erkenntnisse zum Zusammenhang von frühkindlichen Verhaltensaufälligkeiten und späterer Jugenddelinquenz (Loeber/Farrington/Waschbusch 1998) sollten von den anderen Disziplinen nicht gänzlich ignoriert werden, selbst wenn diese Erkenntnisse quer zu den eigenen (teils vorwissenschaftlichen) Präferenzen liegen. Andererseits können Phänomene wie der starke Rückgang der Mordraten in den USA innerhalb weniger Jahre nach 1993 oder der gegenläufige Trend in Russland nach 1989 natürlich nicht mit frühkindlichen Entwicklungsstörungen, sondern nur mit makro- gesellschaftlichen Veränderungen erklärt werden, was wiederum von Psychologie und Psychiat- rie anzuerkennen ist (Loeber/Pardini 2009).

Viele Theorieansätze sind daher zu Recht auf begrenzte Phänomenbereiche zugeschnitten und erheben gar nicht den Anspruch, alle Formen von Kriminalität zu erklären. Zu solchen „Theorien mittlerer Reichweite“ zählen auch einige klassische kriminalsoziologische Theorien wie die Subkulturtheorien (Cohen 1961 [1955]; Miller 1958) und die Theorie der sozialen Desorganisation (Shaw/McKay 1969 [1942]), die sich auf die Erklärung von Jugendkriminalität in problematischen Wohnquartieren der Großstädte konzentrieren. Jedoch verwenden sie dabei meist Bausteine anderer Theorien, die mit einem höheren Allgemeingültigkeitsanspruch auftreten, wie zum Beispiel Lern- oder Kontrolltheorien, die im Prinzip alle Arten von Kriminalität erklären möchten. Ein weiteres Kriterium für die Beurteilung von Kriminalitätstheorien ist daher deren Reichweite bzw. Allgemeingültigkeit.

Theorien mit einem sehr hohen Allgemeingültigkeitsanspruch versuchen zudem meist, sparsame Erklärungen anzubieten, die sich auf einen als wesentlich erachteten Wirkungsmechanismus konzentrieren, und lehnen multifaktorielle Erklärungsansätze ab, die viele unterschiedlichste Einflüsse nebeneinander gelten lassen, wenn sie empirisch als bedeutsam erscheinen. In der Kriminalsoziologie haben vor allem Edwin H. Sutherland (1883-1950) und Travis Hirschi (geb. 1935) „große“ Theorie-Entwürfe, nämlich die Lern- bzw. Kontrolltheorie, entwickelt und damit die Entwicklung des Fachs nachhaltig beeinflusst. Die folgenden Abschnitte konzentrieren sich auf diese und wenige weitere Ansätze und können eine systematische und ausführliche Behandlung von Kriminalitätstheorien nicht ersetzen, die im deutschsprachigen Raum außer von Lamnek $(2007,2008)$ auch von Albrecht (2002, 2009), Dollinger/Raithel (2006), Eifler (2002), Killias (2002) und Lüdemacher/Ohlemacher (2002) angeboten werden. Ebenso wenig kann dieser Beitrag einen auch nur annähernd vollständigen Überblick über empirische Forschungserkenntnisse zur Erklärung von Delinquenz und Kriminalität bieten. Daher greife ich im Anschluss an die Erörterung der „klassischen“ und „neuen“ Theorieansätze nur zwei Gegenstandsbereiche heraus, die in der aktuellen Kriminalitätsforschung einen großen Stellenwert haben.

\section{2 „Klassische“ Theorieansätze}

Lern- und Kontrolltheorie bilden zusammen mit der historisch noch älteren Anomie-Theo- rie bis heute den von der US-amerikanischen Forschung dominierten „Mainstream“ der Kriminalsoziologie. In dem aktuellen Sammelband „Taking Stock. The Status of Criminological Theory“ (Cullen/Wright/Blevins 2006) werden sie als das „Herz“ der kriminologischen Theorien bezeichnet, und in einer Umfrage bei den Mitgliedern der American Society 
of Criminology stehen Lern- und Kontrolltheorie (jedoch nicht die Anomietheorie) immer noch oben auf der Popularitätsliste (Ellis/Cooper/Walsh 2008). Alle drei Ansätze verorten die Ursachen von Kriminalität in den sozialen Positionen und sozialen Beziehungen der Täter, die mehr oder weniger als Produkt ihrer Umwelt gesehen werden. Insofern entsprechen diese Theorien dem gesellschaftspolitischen Zeitgeist Mitte des 20. Jahrhunderts und dem Grundansatz der wohlfahrtsstaatlichen Kriminalpolitik (siehe Abschnitt 4.3) Jedoch gehen die drei Ansätze von unterschiedlichen Menschenbildern aus (Brown/Esbensen/Geis 2001: 410): Nach der Anomietheorie haben die Menschen „gute“, d. h. normkonforme Absichten, werden jedoch durch Frustrationserfahrungen zum Normbruch gebracht, die Lerntheorie sieht die menschlichen Handlungsintentionen als unbestimmt und sehr beeinflussbar an, und die Kontrolltheorie traut jedem Menschen deviante Neigungen zu, die durch Bindungen an die konventionelle Gesellschaft unter Kontrolle gehalten werden müssen.

\section{Anomietheorie}

Die Anomietheorie als eine makrosoziologisch ausgerichtete Theorie setzt an dem grundlegenden Zusammenhang von sozialer Ungleichheit und Kriminalität an und wurde in ihrer klassischen Version von Robert K. Merton (1938) in Anschluss an Durkheim formuliert. Merton sah in der Kriminalität eine von verschiedenen Reaktionsformen auf die psychologische Belastung, die durch den Widerspruch zwischen allgemein als erstrebenswert akzeptierten Zielen eines hohen materiellen Lebensstandards einerseits und der strukturell verankerten sozialen Ungleichheit andererseits entsteht. Denn die Unterprivilegierten haben unter diesen Rahmenbedingungen keine Chance auf die Realisierung des ideologisch vorgegebenen „American Dream“ (Messner/Rosenfeld 1994). Welche Faktoren darüber entscheiden, welche Art von Reaktion aus dieser Frustration folgt - Kriminalität oder eher Apathie oder Rebellion -, thematisierte Merton in seiner Fokussierung auf die gesellschaftliche Makroebene jedoch nicht. Die sozialstrukturelle Dimension der Anomietheorie wurde von den in den 1950er und 1960er Jahren dominanten Subkulturtheorien (Cohen 1961; Cloward/Ohlin 1960) aufgegriffen und mit lerntheoretischen Ansätzen der Ausbreitung delinquenter Normen und den Zugangsmöglichkeiten zu kriminellen Aktivitäten verbunden, die man in erster Linie bei den Jugendgangs in den großstädtischen Unterschichtsvierteln gegeben sah.

Auch wenn die Konzentration von Kriminalität, insbesondere von schwerer Gewaltkriminalität, auf sozialstrukturell benachteiligte Stadtviertel bis heute eine nicht zu übersehende soziale Realität ist, endete die Dominanz der klassischen Anomie- und Subkulturtheorien in den 1970er und 1980er Jahren zugunsten der Kontrolltheorie und anderer Ansätze, die eher die Schichtunabhängigkeit von Kriminalität betonten (siehe Abschnitt 5.4). Die Weiterentwicklung der Anomietheorie verlief in mehreren Bahnen: Zum einen erweiterte Robert Agnew (1992, 1995, 2006) sie zu einer eher individualpsychologischen „General Strain Theory", die schichtunabhängig alle denkbaren Formen von Stress und Frustrationen, die sich aus dem Nichterreichen begehrter Ziele oder aus dem Verlust positiver Stimuli oder dem Auftreten negativer Stimuli ergeben, als Ursache für Delinquenz anerkennt. Zum anderen griffen Steve Messner und Richard Rosenfeld (1994, 2006; Messner 2004; Messner/Thome/Rosenfeld 2008) den sozialstrukturellen Ansatz Mertons erneut auf und bauten ihn unter dem Namen „Institutional Anomie Theory“ in Hinblick auf die kulturellen und 
politischen Institutionen aus, die an der gesellschaftlichen Regulation des Ungleichgewichts von Zielen und Mitteln, diese zu erreichen, mitwirken.

Ihr Hauptargument ist, dass Anomie umso mehr zu Kriminalität führt, je mehr ,the logic of the marketplace intrudes to other realms of social life“ (Messner 2004: 99), d. h. je mehr die Balance zwischen gesellschaftlichen Institutionen von der Marktwirtschaft und ihrer auf materiellem Selbstinteresse und Konkurrenz basierenden Werte dominiert wird. Länder, in denen ein starker Wohlfahrtsstaat das marktliberale Wirtschaftssystem eindämmt und die Menschen durch „Dekommodifizierung“ (Esping-Anderson 1990) vor den materiellen Risiken der Marktwirtschaft schützt, haben demnach niedrigere Kriminalitätsraten zu erwarten. Diese spezifischen Annahmen der Institutional Anomie Theory ließen sich jedoch bislang empirisch nur teilweise bestätigen. Zudem kann sie aufgrund der ausschließlichen Betrachtung der Makroebene ebenso wenig wie Mertons Anomietheorie erklären, wie sich gesellschaftliche Prozesse auf den Lebensalltag auswirken und warum manche Menschen darauf mit kriminellem Verhalten reagieren, andere jedoch nicht (Eisner/Ribeaud 2003: 186).

Eine tragende Rolle spielt die Anomietheorie in ihrer urprünglichen Durkheimschen Fassung auch in der Desintegrationstheorie Heitmeyers (1994, 1997; Anhut/Heitmeyer 2009; Heitmeyer et al. 1996), nach der der soziale Wandel seit längerem einen Verlust an gesellschaftlicher Integration und Regulation und eine zunehmende Individualisierung und Betonung von Konkurrenzprinzipien mit sich bringt. Für die Verlierer dieses Wandels drohen jedoch Anerkennungsdefizite, die in ein steigendes Gewaltpotenzial münden können. In einer ganz ähnlichen Perspektive bringt auch Helmut Thome (2004, 2005, 2007; Thome/ Birkel 2007) den Anstieg der Gewaltkriminalität in den vergangenen Jahrzehnten mit einem zunehmenden Überwiegen des „,egoistischen“ über den „kooperativen“ Individualismus in Verbindung, den Durkheim als Kernproblem einer anomischen Gesellschaft hervorgehoben hatte. Auch bei diesen neueren anomietheoretischen Ansätzen bleiben die empirische Überprüfung und insbesondere die Verbindung zwischen gesellschaftlicher Makroebene und der Mikroebene individuellen Verhaltens problematisch (Albrecht 2002: 796; Rippl/Baier 2005).

Lerntheorien

Der Kerngedanke des von Edwin Sutherland als „differenzielle Assoziation“ bezeichneten lerntheoretischen Ansatzes lautet, dass Kriminalität ebenso erlernt werden würde wie jedes andere soziale Verhalten auch, und dass ,a person becomes delinquent because of an excess of definitions favourable to violation of law over definitions unfavorable to violations of law“ (Sutherland/Cressey 1960: 78). Die Allgemeingültigkeit der Regel, dass Menschen sehr stark durch ihre soziale Umwelt geprägt werden, unterstrich Sutherland dadurch, dass er sie nicht nur auf jugendliche Bandenmitglieder, sondern auch auf Wirtschaftsmanager und ihre „Weiße-Kragen“-Kriminalität anwendete (Sutherland 1945). Spätere Versionen dieses Ansatzes, der von Sutherlands Schülern Robert Burgess und vor allem Ronald Akers (1998; Akers/Jensen 2003, 2006; Burgess/Akers 1966) weiterentwickelt wurde, legten den Schwerpunkt mehr auf den Aspekt der Verstärkung (reinforcement) von Verhaltenstendenzen und verwiesen auf die schon aus der Anomietheorie bekannten sozialstrukturellen Benachteiligungen als Grundlage für delinquentes Verhalten. Denn die offenkundige Schwä- 
che der Theorie der Differenziellen Assoziation liegt darin, dass sie zwar erklären kann, wie delinquente Einstellungen und Handlungen in sozialen Interaktionen von bereits delinquenten auf andere, noch nicht gleichermaßen delinquente Personen übertragen werden, nicht aber, wie diese delinquenten Einstellungen und Handlungen „ursprünglich“ entstehen (Lamnek 2007: 222).

Empirische Unterstützung erhält der Ansatz der Differenziellen Assoziation vor allem durch einen der stärksten und robustesten Zusammenhänge in der Forschung zu Jugenddelinquenz, nämlich der engen Beziehung zwischen der Delinquenz eines Jugendlichen und der Delinquenz seiner Freunde sowie der Bedeutung von Gleichaltrigengruppen und Gangs für kriminelles Verhalten (Klein/Maxson 2006; Klein/Weerman/Thornberry 2006; Warr 2002). Die kausale Bedeutung dieses Zusammenhanges ist jedoch seit langem umstritten, der These der Beeinflussung durch delinquente Freunde (peer pressure) wurde von Kritikern die gegenläufige These von der Selbstselektion bereits delinquenter Jugendlicher in gleichgesinnte Freundesgruppen oder zumindest die Gleichzeitigkeit delinquenter Neigungen und der Orientierung an delinquenten Freunden entgegengehalten (Gottfredson 2006: 91; Hirschi 1969: 135; Sampson 1999).

Eine umfangreiche und methodisch aufwändige Längsschnittforschung, die zunehmend auch Netzwerkanalysen nutzt, hat diese wichtige Frage inzwischen teilweise aufklären können. Der in Selbstberichtsstudien gefundene Zusammenhang zwischen der eigenen Delinquenz eines befragten Jugendlichen und der Delinquenz der Freunde ist zwar deutlich schwächer als früher angenommen und hängt zudem von einer Reihe von weiteren Fakto- ren ab (Haynie 2001, 2002; Haynie/Osgood 2005; Weerman/Smeenk 2005; Weerman/ Bijleveld 2005), aber dennoch lassen sich Kausalwirkungen im Sinne einer weiteren Verstärkung und Intensivierung der Delinquenz durch soziale Interaktionen mit delinquenten Freunden und in Cliquen aufzeigen (Bendixen/Endresen/Olweus 2006; Bernburg/Krohn/Rivery 2006; Espelage/Holt/Henkel 2003; Matsueda/Anderson 1998; Thornberry 1996; Thornberry et al. 1994, 2003). Diese aktuelle Forschung zeigt, dass die Dimension sozialer Interaktionen und Gruppenprozesse auch weiterhin eine wichtige Rolle bei der Analyse von delinquentem Verhalten vor allem bei Jugendlichen spielen werden, zumal damit auch weitere Aspekte wie zum Beispiel die Rolle von Gelegenheitsstrukturen und Routineaktivitäten (Mahoney/Stattin/Lord 2004; Osgood/Anderson 2004; Svensson/Oberwittler 2010) und von räumlich konzentrierter sozialer Benachteiligung (Harding 2008, 2009; Oberwittler 2007, 2008) berührt werden.

Eine psychologische Variante der Lerntheorie, die sich auf die Erklärung von Aggression konzentriert, hat Albert Bandura (1973) formuliert. Mit dem Begriff „Lernen am Modell“" hat Bandura Mechanismen des Nachahmens aggressiven Verhalten durch Vorbilder beschrieben, die vor allem bei der Übertragung gewaltförmigen Verhaltens von Eltern auf ihre Kinder eine Rolle spielen (Baier et al. 2006; Gershoff 2002; Moffitt/Caspi 2003; Pfeiffer et al. 1998). Diese bedeutenden Erkenntnisse haben unter anderem zum gesetzlichen Gewaltverbot in der Kindererziehung beigetragen, das in Deutschland im Jahr 2000 in Kraft trat 


\section{Kontrolltheorie}

Anstatt wie die bisher vorgestellten Theorien nach Ursachen und Motivationen zu suchen, warum Menschen kriminell werden, dreht die Kontrolltheorie die Perspektive herum und fragt, welche Faktoren Menschen daran hindern, ihren Impulsen und Begierden ohne Rücksicht auf Normen zu folgen. Das Potenzial zum abweichenden Verhalten selbst ist nach diesem Ansatz nicht erklärungsbedürftig, da es Nicht-Delinquente ebenso wie Delinquente

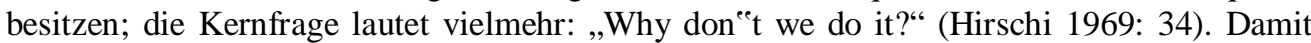
werden bereits die rationalen und berechnenden Komponenten menschlichen Verhaltens ins Blickfeld gerückt, deren Fehlen in der später von Hirschi und Gottfredson formulierten Theorie der niedrigen Selbstkontrolle zentral ist (Abschnitt 5.3).

In seinem bis heute einflussreichen Buch „The Causes of Delinquency“ (1969) beantwortete Hirschi diese Kernfrage mit der Kontrollwirkung von vier verschränkten Elementen sozialer Bindungen an die konventionelle Gesellschaft: „Attachment“, die emotionale Verbundenheit mit anderen Personen; „,commitment“, der persönliche Einsatz für Ziele in der konventionellen Gesellschaft; ,involvement“, die Beschäftigung mit konventionellen Aktivitäten; und ,belief“, der Glaube in die moralische Gültigkeit konventioneller Normen. Die zentrale Annahme der Kontrolltheorie ist, dass derjenige, der starke Bindungen an die konventionelle Gesellschaft hat (,stakes in conformity“), etwa durch eine enge Beziehung zu Lehrern und Lehrerinnen oder durch einen hohen Berufsstatus (oder die Aussicht, diesen zu erreichen), durch delinquentes Verhalten, welches negative Sanktionen seitens des sozialen Umfelds nach sich ziehen würde, mehr zu verlieren hätte als jemand, dem diese Bindungen fehlen. Wer nichts zu verlieren hat, ist im Umkehrschluss frei, gegen die Regeln der Gesellschaft zu verstoßen.

Hirschis Kontrolltheorie hat seit den 1970er Jahren eine Vielzahl von empirischen Studien zur Jugenddelinquenz beeinflusst, in denen sich starke Zusammenhänge zwischen den Bindungen gegenüber Eltern und Schule sowie dem Ausmaß konventioneller Aktivitäten und Überzeugen einerseits und der selbstberichteten Delinquenz andererseits zeigten. Allerdings fielen diese Zusammenhänge in komplexeren empirischen Modellen, die zusätzlich zu den Bindungs-Variablen auch die Beziehungen zu delinquenten Gleichaltrigen enthielten und damit Kontroll- und Lerntheorie gegeneinander testen können, teils deutlich schwächer aus (Albrecht 2002: 786; Kubrin/Stucky/Krohn 2009: 282). Die lange Zeit spürbare Unterbewertung der Rolle der delinquenten Gleichaltrigen zugunsten der von Hirschi bevorzug- ten ausschließlich kontrolltheoretischen Perspektive hat sich als nicht sinnvoll erwiesen und ist in der Jugenddelinquenzforschung mittlerweile überwunden worden. Eine wichtige Bestätigung hat der kontrolltheoretische Grundgedanke dagegen in Gestalt der „Age-graded Theory of Informal Social Control“ im Rahmen der Lebenslaufforschung zur Entwicklung krimineller Karrieren erhalten (Laub/Sampson 2003; Sampson/Laub 2005; siehe Abschnitt 5.5).

Abschließend lässt sich mit Blick auf alle drei „klassischen“ kriminalsoziologischen Theorieansätze feststellen, dass sich der von ihren Hauptvertretern eingeforderte Exklusivitätsanspruch überlebt hat. Jede Theorie beschreibt eine bedeutsame Perspektive auf die Ursachen von abweichendem Verhalten, die aber für sich nicht die Komplexität sozialen Verhaltens abbilden können. Der Trend in der empirischen Kriminalitätsforschung geht trotz entgegengesetzter Versuche eher hin zu einer Kombination und Integration unterschiedli- 
cher Erklärungsansätze (Agnew 2005: 311; Kubrin/Stucky/Krohn 2009: 247; Wikström/ Sampson 2006). Aber auch traditionsreiche kriminalsoziologische Theorien wie die oben erwähnten Subkulturtheorien und die Theorie der sozialen Desorganisation von Shaw und McKay (1969) verknüpften bereits Grundgedanken von Anomie-, Lern- und/oder Kontrolltheorie. Eine puristische Abgrenzung zwischen diesen Theorien fällt auch deswegen schwer, weil sie vielfach dieselben empirischen Phänomene, wie zum Beispiel ein belaste- tes Eltern-Kind-Verhältnis, unterschiedlich interpretieren. Was die drei klassischen Theo- rien trotz dieser Unterschiede miteinander verbindet, ist ein ,übersozialisiertes Konzept“ (Wrong 1961) des Menschen in der Tradition Durkheims und Parsons, das abweichendes Verhalten vorrangig als Produkt sozialer Rollen und Bindungen sieht und die Perspektive individueller Handlungsmacht (,agency“) ebenso unterbelichtet wie die Bedeutung von Situationen.

\section{3 „Neue“ Theorieansätze}

Die eben genannten Lücken bei den „klassischen“ kriminalsoziologischen Theorien versuchen neuere Erklärungsansätze aufzufüllen, die individuelle Handlungsentscheidungen in den Mittelpunkt stellen und damit der grundlegenden Annahme der Willensfreiheit insoweit folgen, als dass Menschen sich trotz prägender Sozialisations- und Umwelteinflüsse in konkreten Situationen für oder gegen einen Normbruch entscheiden können und auch müssen (Eifler 2009; Hess/Scheerer 1997: 110). Die Entwicklung der kriminalsoziologischen Theorien folgte damit der zunehmenden Bedeutung des methodischen Individualismus in der Soziologie insgesamt (Coleman 1986, 1990; vgl. Lüdemann 2000). Aber auch die Bedeutung von psychologischen und neurowissenschaftlichen Erklärungsansätzen ist gewachsen, die die intra-individuellen Mechanismen von Handlungsentscheidungen aus ihrer jeweiligen disziplinären Sicht untersuchen - bis hin zur provokanten Negierung der Willensfreiheit (Prinz 2008; Roth 2006) - und die abweichendes Verhalten oftmals als Ausdruck mehr oder weniger schwerwiegender psychischer Defizite der Täter deuten.

Diese Erklärungsansätze gibt es natürlich schon seit langem, ebenso wie die „klassischen" kriminalsoziologischen Theorien durch sie nicht plötzlich entwertet werden. Jedoch ist eine Verschiebung in den Gewichten zwischen den „,klassischen“ und ,neuen“ Ansätzen zu beobachten, die Garland (1999; 2001: 182 ff.) in den Kontext veränderter gesellschaftsund kriminalpolitischer Rahmenbedingungen in der „Spätmoderne“ gestellt hat (siehe Abschnitt 4.3). Garland erkennt in den neueren kriminologischen Theorien zwei parallele, jedoch sehr unterschiedliche Tendenzen, die Kriminalität entweder als banales Alltagsgeschehen (,criminologies of everyday life“) oder aber als potenziell gefährliche Psychopathologie (,criminology of the dangerous other") deuten, in jedem Fall aber den bis in die 1970er vorherrschenden „,wohlfahrtsstaatlichen“ Grundkonsens verlassen, Kriminalität in erster Linie als Folge von Benachteiligungen und Sozialisationsdefiziten zu verstehen.

Rational Choice-Ansatz

Die bekannteste Entscheidungstheorie ist zugleich die umstrittenste, zumal im Kontext von Kriminalität: Der Rational Choice-Ansatz geht davon aus, dass Menschen Nutzen und Kosten von Handlungsalternativen zweckrational abwägen und nach dem Kriterium der egoistischen Nutzenmaximierung handeln (Cornish/Clarke 1986; Eifler 2002; Fetchenhauer 1998; 
Karstedt/Greve 1996; Lüdemann/Ohlemacher 2003: 51). Diese Vorstellung schließt direkt an das utilitaristische und hedonistische Menschenbild der Aufklärung und Strafrechtstheoretiker wie Cesare Beccaria und Jeremy Bentham an (Gottfredson/Hirschi 1990: 3-14; Lamnek 2007: 64). Offensichtlich ist zweckrationales Handeln jedoch bei vielen Formen der Kriminalität nicht erkennbar (de Haan/Vos 2004; Katz 1988).

Hatte der Wirtschaftswissenschaftler Gary Becker diesen Entscheidungsprozess noch als eine rein ökonomische Abwägung beschrieben (,,a person commits an offense if the expected utility exceeds the utility he could get by using his time and other resources at other activities“, Becker 1968: 176), so nahmen spätere Versionen der Rational-ChoiceTheorie dieser berechtigten Kritik am kruden Rationalitätsbegriff durch die flexibleren Konzepte des „subjektiven erwarteten Nutzens“ (subjective expected utility, SEU) zwar den Wind aus den Segeln, setzten sich damit aber auch dem Vorwurf der mangelnden Falsifizierbarkeit aus, da bei beinahe jeder Handlungsentscheidung angenommen werden kann, dass sie zumindest einer subjektiven, objektiv schwer zugänglichen Nutzenerwartung folgt (Vanberg 1993: 95). Sogar Selbstmordattentate lassen sich so scheinbar „rational“ erklären (Atran 2003; Caplan 2006; Legewie/Simons 2009).

Wesentlich hilfreicher erscheinen die Erweiterungen der Rational-Choice-Theorie durch „Skripte“ (Handlungsanweisungen) und „Frames“ (Situationsdefinitionen), die fertig abrufbar sind und in Entscheidungssituationen an die Stelle von bewussten Kosten-NutzenAbwägungen treten oder diese zumindest stark beeinflussen können (Esser 2005; Kroneberg 2005). Kulturelle und moralische Präferenzen können so gleichberechtigt neben egoistischen Motiven als Einflüsse auf Entscheidungen anerkannt werden. Der Rational ChoiceAnsatz, der dann eher eine allgemeine Entscheidungstheorie ist, untersucht also, wie das Handeln von Präferenzen, aber auch von anderen, z. B. situationsbezogenen Faktoren beeinflusst wird, während die „klassischen“ soziologischen Theorien nach den Wurzeln eben dieser Präferenzen suchen, aber die Handlungssituation unterbelichten (McCarthy 2002: 423). Aus der Perspektive der Erforschung sozialer Probleme ist die „klassische“ Fragestellung nach der Herkunft von Präferenzen häufig wichtiger (vgl. Boudon 1998: 182); dennoch können sich beide Fragestellungen in integrativen Theorieansätzen durchaus ergänzen (siehe unten).

Die Stärke der entscheidungstheoretischen Ansätze liegt vor allem in der Fokussierung auf situationsbezogene Faktoren krimineller Handlungen. Dazu gehört in einem weiteren Sinne auch die wichtige Frage der Abschreckungswirkung von Sanktionen. Nach dem hedonistischen Menschenbild des Rational Choice-Ansatzes kalkulieren potenzielle Täter erwartete Nutzen und Kosten eines Normbruchs und entscheiden sich für den Normbruch, wenn er sich „lohnt“. Diese Sichtweise erinnert an die Kontrolltheorie, die ebenfalls nicht an den Motivationen für, sondern an den Hinderungsgründen gegen abweichendes Verhal- ten interessiert ist. Kosten von Normbrüchen entstehen im Wesentlichen durch die Andro- hung von informellen und formellen Sanktionen sowie durch Schamgefühle, die als interna- lisierte negative Sanktionen verstanden werden können (Eifler 2009: 34).

Empirische Studien zur Abschreckungswirkung von Sanktionen haben gezeigt, dass erstens informelle Sanktionen durch nahestehende Personen wichtiger sind als staatliche Strafsanktionen (was deren Existenz allerdings nicht überflüssig macht), dass zweitens die Schwere von formellen Sanktionen einen deutlich geringeren Einfluss auf das Handeln hat 
als die Gewissheit, mit der überhaupt eine formelle Sanktion erfolgt, und dass drittens andere Überlegungen, wie z. B. die wahrgenommene „Coolness" des Normbruchs, wichtiger sein können (Grasmick/Bursik 1990; Matsueda/Kreager/Huizinga. 2006; Nagin 1998; Pratt et al. 2006).

Zur empirischen Analyse der Abschreckungswirkung von Strafsanktionen wurden zwei quasi-experimentelle Methoden angewendet, die am ehesten geeignet sind, Kausaleffekte von bloßen korrelativen Zusammenhängen zu unterscheiden: Zum einen wurden in Befragungen mithilfe des so genannten "faktoriellen Designs“ hypothetische Situationen (Vignetten) hinsichtlich der Attraktivität des Nutzens, der Entdeckungswahrscheinlichkeit oder der Strafhöhe einer Straftat variiert und die Befragten nach ihren Verhaltensintention befragt (Kronenber/Heintze/Mehlkop 2008; Thurman 1989). Unterschiede in der hypothetischen Bereitschaft, unter einen jeweiligen Bedingungen einen Normbruch zu begehen, lassen sich aufgrund der Zufallsverteilung der Vignetten kausal interpretieren, wenn man den Verhaltensintentionen eine Relevanz in Hinblick auf tatsächliches Verhalten zubilligt (Dülmer 2007; Eifler 2007; Wallander 2009).

Zum anderen haben Wirtschaftswissenschaftler anhand von räumlich aggregierten Zeitreihen überwiegend offizieller Kriminalitätsdaten sowie weiterer sozialstruktureller Informationen einen Effekt von polizeilichen Aufklärungsquoten und Strafsanktionen auf die registrierte Kriminalität gefunden. Demnach haben hohe Aufklärungsquoten einen reduzierenden Effekt auf die weitere Kriminalitätsentwicklung (Levitt 1997, 1998; Mocan/Rees 2005). In einer neuen deutschen Studie fand Entorf (2008) darüber hinaus auch einen steigernden Effekt der Diversionsrate (staatsanwaltschaftliche Einstellung evtl. mit informellen Auflagen) gegenüber der Rate formeller Gerichtsverfahren auf die Höhe der registrierten Jugendkriminalität, was den bisherigen kriminologischen Erkenntnissen über die Sanktionswirkungen bei Jugendkriminalität widerspricht (Walter 2001: 277). Die Ergebnisse dieser ökonometrischen Studien müssen jedoch vorsichtig bewertet werden, da zwar die statistischen Verfahren sehr aufwändig sind, jedoch das hohe räumliche Aggregationsniveau und die Herkunft der Daten aus der polizeilichen Kriminalstatistik letztlich keine Aussagen über die Mikro-Ebene der handelnden Personen und die dort wirksamen Mechanismen erlauben.

Routine Activities Approach

Der „Routine Activities Approach“ ist eine Variante des Rational Choice-Ansatzes, die mit den Tatgelegenheiten die „Angebotsseite“ der Kriminalität in den Mittelpunkt stellt und zu Recht als „Kriminologie ohne Täter“ bezeichnet wurde (Sessar 1997). Diese Theorie sieht Kriminalität als Begleiterscheinung alltäglicher Routinehandlungen und wird besonders für die Erklärung räumlicher und zeitlicher Verteilungen von Eigentums- und Gewaltdelikten und der Rolle situativer Einflüsse genutzt (Felson 1994; Felson/Clarke 1998). Der Kerngedanke ist, dass Kriminalität, soweit sie einen direkten Kontakt zwischen Täter und Opfer bzw. Objekt bedingt, zwingend das raum-zeitliche Zusammentreffen dreier Elemente voraussetzt: „An offender with both criminal inclinations and the ability to carry out those inclinations, a person or object providing a suitable target for the offender, and absence of guardians capable of preventing violations" (Cohen/Felson 1979: 590) Auch wenn also delinquente Neigungen der Täter durchaus erwähnt werden, so richtet der Routine Activities Approach seine Aufmerksamkeit doch vollständig auf das Kosten-Nutzen-Verhältnis zwi- 
schen attraktiven Zielen und effektiver Kontrolle. Cohen und Felson argumentierten, dass der massive Anstieg der Eigentumsdelikte in den wirtschaftlichen Boomzeiten der 1960er und 1970er Jahre nicht mit einer Verschärfung sozialer Probleme, sondern eher mit der Zunahme attraktiver Ziele (z. B. leicht zu transportierender elektronischer Geräte) und einem Nachlassen wirksamer Kontrollen (z. B. in Selbstbedienungsgeschäften oder unbewachten Wohnungen) erklärt werden könne. Zentral und in Hinblick auf die Kriminalpolitik folgenreich ist die Annahme des Routine Activities Approach, dass sich Kriminalitätshäufigkeiten durch die Veränderung der beiden kausalen Faktoren Gelegenheiten und Kontrolle - auch ohne Berücksichtigung des dritten Faktors „motivierte Täter“ - beeinflussen ließen (Felson/ Clarke 1998). Diese umstrittene Annahme schafft die Basis für situationsbezogene Ansätze der Kriminalprävention, die auf eine Intensivierung informeller und formeller (polizeilicher) Überwachung sowie auf technische Sicherungs- und Überwachungssysteme setzt.

Empirische Studien zur räumlichen Kriminalitätsverteilung und Opferbefragungen haben die Annahmen des Routine Activities Approach insofern bestätigt, als Kriminalitätsrisiken deutlich mit Lebensstilen wie z. B. der Präferenz außerhäusiger Freizeitbeschäftigungen (Hindelang/Gottfredson/Garofalo 1978; Kennedy/Forde 1990) und entsprechenden Gelegenheitsstrukturen wie z. B. innerstädtischer Vergnügungsbezirke zusammenhängen (Roncek/Maier 1991; Rountree/Land/Miethe 1994; Sherman/Gartin/Buerger 1989; Wikström 1991). Allerdings deuten einige Untersuchungen darauf hin, dass Indikatoren sozialer Benachteiligungen und damit ,klassische“ Theorieansätze meist eine verglichen mit den Gelegenheitsstrukturen größere Erklärungskraft haben (vgl. Oberwittler 2004b; Pratt/Cullen 2005). Experimentelle Studien konnten allerdings zeigen, dass Kriminalitätshäufigkeiten in besonders belasteten städtischen Räumen durch die Intensivierung von Polizeikontrollen (Hot Spots Policing) zurückgehen, ohne dass es zu einem Verlagerungseffekt in benachbarte Gebiete kommt (Braga 2005; Sherman/Weisburd 1995). Auch sind teils deutliche Anstiege von Raub- und Gewaltdelikten während Polizeistreiks nachgewiesen worden, die als natürliche Experimente interpretiert werden können (Sherman 1997: 8/5).

Theorie der niedrigen Selbstkontrolle (,General Theory of Crime“)

Kein anderer Theorieentwurf hat in der Kriminalitätsforschung der letzten rund zwanzig Jahre eine so starke Resonanz hervorgerufen wie die Theorie der niedrigen Selbstkontrolle (,low self control“) von Michael Gottfredson und Travis Hirschi (1990). Obwohl diese selbst erklärte „General Theory of Crime“ mit der Fokussierung auf Defizite in der Verhaltenssteuerung einen sehr wichtigen (wenn auch keineswegs neuen) Aspekt der Genese abweichenden Verhaltens auf der Ebene individueller Unterschiede aufgegriffen und die Entwicklung der Kriminalitätsforschung stark geprägt hat, konnte sie sich mit ihrem Allgemeingültigkeitsanspruch und ihren spezifischen Annahmen über Entstehung und Stabilität von Selbstkontrolle letztlich nicht durchsetzen.

Im Kern besagt diese Theorie, dass Selbstkontrolle eine stabile, in den ersten Lebensjahren durch Erziehung vermittelte Verhaltensdisposition ist, die Menschen dazu befähigt, die langfristig negativen Folgen von Handlungen zu überblicken und ihre Impulse zu zügeln. Menschen mit niedriger Selbstkontrolle erliegen den ,Versuchungen des Augenblicks“, haben eine „Hier und jetzt"-Orientierung und eine Vorliebe für riskante und körperliche Aktivitäten (Gottfredson/Hirschi 1990: 86 ff.). Da viele Formen der Kriminalität 
ebenso wie andere „unbesonnene“ Handlungen eine unmittelbare Befriedigung von Impulsen ohne Rücksicht auf zukünftige negative Auswirkungen darstellen, neigen Menschen mit niedriger Selbstkontrolle nach dieser Auffassung nicht nur zu Straftaten, sondern auch zu Unfällen und anderen Formen selbstschädigenden Verhaltens. Die Theorie der niedrigen Selbstkontrolle baut insofern auf ,klassische“ Kriminalitätstheorien der Aufklärung und Rational Choice-Ansätzen auf als sie Kriminalität als Resultat der Unfähigkeit von Straftätern erklärt, rational zwischen den kurzfristigen Verlockungen eines Normbruchs oder in anderer Weise riskanten Handlungen und deren langfristigen Nachteilen durch Sanktionen oder andere Gefahren abzuwägen.

Viele Studien haben enge Zusammenhänge zwischen der von Grasmick et al. (1993) entwickelten umfangreichen Skala, die verschiedene Teildimensionen der Selbstkontrolle messen soll, und selbstberichteter Delinquenz gefunden und der Theorie damit empirische Unterstützung gegeben (Gottfredson 2006; Pratt/Cullen 2000; Vazsonyi et al. 2001, 2004). Insbesondere die Teildimension der Risikoorientierung (risk seeking) zeigt starke Korrelationen mit delinquentem und selbstschädigendem Verhalten (Ribeaud/Eisner 2006). Eine Korrelation von straffälligem Verhalten, Unfällen und gesundheitsschädlichen Verhaltenswiesen haben verschiedene Studien berichtet (Junger/Tremblay 1999; Quensel 2009). Diese Erkenntnisse über die Generalität von Risikoverhalten sind jedoch keineswegs neu (Hurrelmann/Lösel 1990; Jessor 1998; Raithel 2001; Zuckerman 1979).

Auch die Bedeutung der Handlungskontrolle bzw. Impulsivität für delinquentes Verhalten wurde in experimentellen Studien bereits lange vor Gottfredson und Hirschi herausgearbeitet (z. B. Lösel 1975; Mischel 1974; Mischel/Shoda/Rodriguez 1989). Kritisch wird auch bewertet, dass Gottfredson und Hirschi niedrige Selbstkontrolle explizit nicht als Persönlichkeitsmerkmal (trait) betrachten und ausschließlich durch unzureichende Erziehungspraktiken in den ersten Lebensjahren verursacht sehen (Beaver et al. 2007, 2008; Pratt et al. 2002). Gegen eine monokausale Rolle bei der Verursachung von Kriminalität sprechen viele Studien, die zeigen konnten, dass niedrige Selbstkontrolle nur unter bestimmten Umständen ein relevanter Prädiktor individueller Delinquenz ist, zum Beispiel bei gleichzeitiger Existenz devianter Einstellungsmuster (Schoepfer/Piquero 2006; Wikström/Treiber 2007) oder delinquenter Freundeskreise (Meldrum/Young/Weerman 2009) oder bei günstigen im Gegensatz zu ungünstigen Tatgelegenheiten (Seipel/Eifler 2004; Wright et al. 2004).

Neurowissenschaftliche Erklärungsansätze

In den letzten Jahren hat die neurowissenschaftliche Erforschung sozialen Verhaltens durch die Entwicklung neuer bildgebender Verfahren, vor allem der funktionellen Magnetresonanztomographie (fMRT, englisch fMRI), sowie der Molekularbiologie und Genomanalyse einen rasanten Aufschwung genommen, dessen Relevanz für das Verständnis menschlichen Verhaltens noch nicht klar ist. Sicher ist jedoch, dass sich die Sozialwissenschaften dieser Entwicklung nicht völlig verschließen werden (Diewald 2010; Freese 2008). Weil neurobiologische und soziologische Weltbilder traditionell antagonistisch wahrgenommen werden und die ätiologische Perspektive auf Kriminalitätsursachen in dieser Disziplin ohnehin kontrovers ist, reagiert die deutschsprachige Kriminalsoziologie auf diese Entwicklung skeptisch bis abwehrend (Böllinger/Jasch/Krasmann 2010; Kreissl/Steinert 2008). Plakative Schlagworte (,Tatort Gehirn“, Markowitsch/Siefer 2007) und Tendenzen zum biologisti- 
schen Determinismus (Prinz 2008; Roth 2006) haben diese Abwehr verstärkt. Der folgende Abschnitt bietet keinen systematischen Überblick, sondern nur einzelne Schlaglichter auf dieses Forschungsfeld und versucht dabei deutlich zu machen, warum es auch aus sozialwissenschaftlicher Sicht interessant sein könnte.

Zunächst zeigen die Erkenntnisse der Neurowissenschaften, dass sich für die kognitiven und emotionalen Grundlagen menschlichen Verhaltens somatische Entsprechungen in der Anatomie und Physiologie des Gehirns finden lassen, ohne dass dadurch bereits eindeutige Kausalitäten hergestellt würden. Für höhere mentale Konzepte wie Vertrauen (Baumgartner et al. 2008), Gerechtigkeitsempfinden (Tricomi et al. 2010) und Schadenfreude (Takahashi et al. 2009) können mit Hilfe von fMRI „zuständige“ Gehirnareale lokalisiert werden. Das neuronale System besteht aus einem komplizierten Geflecht von spezialisierten Gehirnregionen und Hormonhaushalten, durch deren Zusammenwirken Emotionen, Kognitionen und Handlungsentscheidungen bewirkt werden. Dabei arbeiten evolutionär ältere Gehirnregionen, vor allem das so genannte Limbische System (unter anderem Amygdala, Hippocampus), und die evolutionär jüngeren Regionen des präfrontalen Kortex (PFK) zusammen. Aufgrund von unfall- oder krankheitsbedingten Ausfällen ist schon länger bekannt, dass einzelne Regionen des PFK für anspruchsvollere Aufgaben wie Moral, Reflexion, Selbstkontrolle und längerfristige Planung verantwortlich sind (Bechara/Damasio/Damasio 2000; Wikström/Treiber 2007).

Eine allgemeine Annahme lautet nun, dass ein Ungleichgewicht des neuronalen Systems zu Ungunsten des PFK mit normverletzenden, riskanten und aggressiven Verhaltensweisen korreliert (Gianotti/Knoch/Faber 2009; Strüber/Lück/Roth 2008). Knoch und Fehr (2007) haben experimentell gezeigt, dass die Inhibition „unvernünftiger“ Versuchungen durch das gezielte Aussschalten des rechten PFK durch kurzfristige magnetische Impulse gestört werden kann. Spitzer el al. (2007) ordnen dieser Gehirnregion auch die Aufgabe der Normbefolgung zu. Sie stellten eine erheblich höhere Stoffwechselaktivität im rechten PFK fest, wenn Untersuchungsteilnehmer wussten, dass egoistisches Verhalten vom Partner eines Ultimatumspiels bestraft werden konnte, als wenn keine Bestrafung möglich war. In einer anderen experimentellen Studie zur aufgeschobenen Bedürfnisbefriedigung mussten Versuchspersonen zwischen einem unmittelbaren, aber niedrigeren monetären Gewinn und einem in der Zukunft liegenden, höheren Gewinn wählen, während ihre Gehirntätigkeit mit fMRI gemessen wurde (McClure et al. 2004). Es zeigte sich eine relativ stärkere Aktivität im Limbischen, für Emotionen und Impulse „zuständigen“ System, wenn sich Versuchspersonen für den unmittelbaren Gewinn entschieden, und eine relativ stärkere Aktivität im für Selbststeuerung und Planung zuständigen PFK, wenn die Entscheidung für den aufgeschobenen, höheren Gewinn fiel. Die Bevorzugung eines unmittelbaren niedrigeren Gewinnes (discounting) zeigt auch in Befragungen einen engen Zusammenhang mit Selbstkontrolle (Wikström/Butterworth 2006).

Die Entwicklung dieser kortikalen Gehirnregionen ist entgegen früherer Auffassungen erst mit etwa 25 Jahren abgeschlossen (Steinberg 2005), und es gibt Hinweise darauf, dass sich durch unterschiedliche Entwicklungsgeschwindigkeiten der limbischen und kortikalen Gehirnareale während der Pubertät und Adoleszenz ein Ungleichgewicht zugunsten eher impulsiver und risikoreicher Verhaltensentscheidungen ergibt (Galvan/Hare/Parra 2006; Giedd 2008). Es fällt auf, dass dieser Altersverlauf in etwa mit der „Age-Crime“-Kurve der 
Kriminalität, aber auch mit dem Unfallrisiko im Straßenverkehr übereinstimmt. Diese Erkenntnisse bieten differenzierte Einblicke in die neuronalen Grundlagen der Verhaltenskontrolle und könnten damit einen relevanten Mosaikstein in der Erklärung delinquenten Verhaltens darstellen. Als monokausaler Erklärungsansatz für Kriminalität eignen sie sich jedoch ebenso wenig wie Gottfredson und Hirschis Theorie der niedrigen Selbstkontrolle.

Ein weiterer von den Neurowissenschaften zurzeit intensiv erforschter Aspekt betrifft die Funktion von Neurotransmittern für aggressives Verhalten. Der Botenstoff Serotonin (auch 5-HT genannt) spielt eine wichtige Rolle in der Regulierung von Emotionen im Zusammenspiel von Limbischem System und PFK (Lee/Coccaro 2007; Seo/Patrick/Kennedy 2008). Eine Störung der Produktion und Rezeption von 5-HT reduziert die Aktivität des PFK und steigert hingegen die Aktivität der Amygdala mit der Folge verstärkter negativer Emotionen und Angstwahrnehmungen sowie einer höheren Wahrscheinlichkeit impulsivaggressiven Verhaltens. Eine solche Störung stellt zum Beispiel eine erbliche Genvariation dar, bei der das für den Hormonstoffwechsel wichtige Enzym MAOA (Monoaminooxidase A) fehlt. Mit den Längsschnittdaten der neuseeländischen „Dunedin Multidisciplinary Health and Development Study“ konnten Caspi et al. (2002) zeigen, dass diese neurobiologische Störung nur dann zu einer erhöhten Gewaltneigung im Erwachsenenalter führt, wenn die betroffenen Personen im Kindesalter selbst Opfer körperlicher Misshandlungen geworden waren, und dass in umgekehrter Perspektive der Zusammenhang zwischen als Kind erfahrener Gewalt und späterer eigener Gewaltneigung erheblich stärker ist, falls das Feh- len von MAOA die „,normale“ neuronale Stressverarbeitung verhindert. Dieser Befund wur- de inzwischen anhand anderer Längsschnittdaten bestätigt (Guo/Roettger/Cai 2008).

Diese Wechselwirkung zwischen Gewalterfahrungen und neurobiologischen Anlagen ist eine der wenigen bislang empirisch belegten Beispiele einer Gen-Umwelt-Interaktion im Bereich des Sozialverhaltens. Gen-Umwelt-Interaktionen (,G x E“) stellen ein zentrales Paradigma der gegenwärtigen neurowissenschaftlichen Forschung dar, nach dem menschliche Sozialisation auf der Basis ,einer kontinuierlichen Wechselwirkung zwischen genetischer Aktivität und Umweltbedingungen“ (Asendorpf 2008; vgl. Kim-Cohen et al. 2004; Moffitt 2005; Rutter/Silberg 2002) stattfindet.

Der Begriff der „neuronalen Plastizität“ beschreibt ebenfalls diese Beeinflussbarkeit der neurobiologischen Grundlagen durch Erfahrungen. Diese Vorstellung ist aus sozialwissenschaftlicher Sicht sehr wichtig, da es einem kruden genetischen Determinismus vorbeugt und die ideologisch geführte Debatte über „Anlage oder Umwelt“ ersetzen kann (Sameroff 2010). Zu der Auffassung, dass die neurobiologische Basis menschlichen Verhaltens durch Gene zwar mit beeinflusst, aber nicht determiniert wird, haben auch neue Erkenntnisse im Bereich der so genannten Epigenetik beigetragen (Depue 2009; Meaney 2010). Durch Umwelteinflüsse können die funktionellen Eigenschaften von Genen modifiziert, einzelne Funktionsbereiche aktiviert und andere „,abgestellt“ werden. Insbesondere durch frühkindliche Umweltbedingungen können die molekularbiologischen Eigenschaften von Zellen dauerhaft beeinflusst werden, wie z. B. eine Studie zu den Auswirkungen von kindlichem Missbrauch auf die neurobiologischen Grundlagen der Stressverarbeitung bei erwachsenen Suizid-Opfern zeigte (McGowan et al. 2009).

Der Eindruck, der sich aus diesen Studien ergibt, spricht weniger für einen biologischen Determinismus als für äußerst komplexe Zusammenhänge miteinander verwobener 
und aufeinander reagierender Ebenen (Hood 2005). Mit der Einbeziehung genetischer und neuronaler Grundlagen menschlichen Verhaltens wird die Bedeutung individueller Unterschiede und Persönlichkeitsmerkmale wesentlich stärker betont als dies in der Soziologie üblich ist (Freese 2008). Zentral ist jedoch, dass individuelle Eigenschaften und soziale Umweltbedingungen miteinander interagieren, mit der Folge, dass Wirkungen sozialer Bedingungen auf Menschen individuell sehr unterschiedlich sind. Die Plastizität des neuronalen Systems, die offenbar vor allem am Beginn des Lebens sehr groß ist, unterstreicht die Bedeutung der frühen Kindheitsphase für die langfristige Entwicklung individuellen delinquenten Verhaltens - und für dessen Prävention. Auf der anderen Seite muss aus sozialwissenschaftlicher Sicht betont werden, dass mit dem Fokus auf individuelle Verhaltensdispositionen nur ein kleiner Ausschnitt der Bedingungen, die soziales Verhalten prägen, erfasst wird.

\section{Integrative Theorieansätze}

Angesichts der unterschiedlichen Begrenzungen und Probleme, von denen keine der genannten Theorien frei ist, haben in den letzten Jahren die Stimmen zugenommen, die integrative Erklärungsansätze von Delinquenz und Kriminalität befürworten. Der Begriff ,integrativ“ bezieht sich zum einen auf die Verbindung verschiedener Disziplinen, in erster Linie Psychologie und Soziologie, und zum anderen auf die Verbindung der Mikroebene individuellen Handelns mit den verschiedenen Meso- und Makroebenen sozialer Beziehungen und Kontexte, in denen Menschen agieren. Der historisch weit zurückreichende und weit verbreitete „Mehrfaktorenansatz“ oder „risk factor approach“, der ebenfalls eine Vielzahl ganz unterschiedlicher Perspektiven berücksichtigt, kann dabei kaum als Vorbild dienen, da er die Bedeutung aller Einflussfaktoren vorrangig auf der Basis statistischer Korrelationen, ohne Annahmen zur kausalen Ordnung und zu den Wirkungsmechanismen, bewertet hat (Lamnek 2008).

Das verbindende Element der integrativen Theorieansätze ist die zu Beginn des Abschnitts 5.2 erwähnte Vorstellung eines sinnhaft agierenden Individuums, das in konkreten Situationen Handlungsentscheidungen treffen muss. Erklärungsansätze, die Kriminalität in der soziologischen Tradition Durkheims lediglich als ein kollektives Phänomen, verursacht durch anonyme Strukturen, verstehen, übersehen, dass gesellschaftliche Phänomene stets durch die Vielzahl individueller Handlungen konstituiert werden. Erklärungsansätze, die Kriminalität ausschließlich als eine individuelle Abweichung aufgrund von Persönlichkeitsoder Erziehungsdefiziten verstehen, übersehen andererseits den Einfluss sozialer Rahmenbedingungen. Diesem Spannungsverhältnis individueller und kollektiver Ebenen versuchen so genannte Mikro-Makro-Modelle gerecht zu werden, die allerdings wegen der unvermeitlichen Komplexität der Zusammenhänge nur mit hohem Aufwand in empirische Forschung umsetzbar sind. In der deutschen Kriminalsoziologie haben sich vor allem Hess/Scheerer (1997, 2004) mit ihrer programmatischen „Skizze einer konstruktivistischen Kriminalitätstheorie" für eine solche komplexe Modellbildung und die Verbindung unterschiedlicher Theorieansätze wie Kontrolltheorie, Routine Activities und Labeling Approach stark gemacht, ohne dieses Programm jedoch auch empirisch umzusetzen.

Aus der empirischen Forschung seien hier nur zwei aktuelle Ansätze - ein eher psychologischer und ein eher soziologischer - herausgegriffen, die für die Bemühungen um eine 
integrative Theoriebildung stehen und die weitere Erforschung der Ursachen von Delinquenz und Kriminalität voranbringen könnten.

Das „Biopsychosocial Model“ von Kenneth A. Dodge (Dodge/Pettit 2003) kombiniert Aspekte der Persönlichkeitsmerkmale und familiärer Prozesse mit Einflüssen des soziokulturellen Kontextes, die insbesondere durch Interaktionen und Bindungen mit Gleichaltrigen vermittelt werden. Den Kern dieses Modells bildet die aus der Theorie des „Social Information Processing“ (Crick/Dodge 1994) stammende Überlegung, dass jegliche konforme oder abweichende Handlung durch kognitiv und emotional geleitete Entscheidungen des Individuums ausgelöst wird. Diese mentalen Prozesse werden als unmittelbare, proximale Ursachen abweichenden Verhaltens angesehen, während den familiären und Peer-Faktoren die Rolle von vermittelnden Einflüssen zukommt und den Persönlichkeitsmerkmalen und soziokulturellen Kontexten eher eine indirekte (distale) Kausalrolle zugeschrieben wird. Entscheidend ist die Überlegung, dass die unterschiedlichen Einflussfaktoren im Gesamtmodell nicht nur additiv, sondern auch multiplikativ wirken, d. h. dass sie sich in ihrer Wirkung in Abhängigkeit von der Ausprägung anderer Faktoren abschwächen oder verstärken können.

Auch die „Situational Action Theory“ von Per-Olof Wikström (2006, 2007b) rückt den individuellen „Wahrnehmungs-Entscheidungs-Prozess“ für oder gegen den Normbruch ins Zentrum und fragt nach den Wechselwirkungen zwischen den Situationen, die sich durch unterschiedliche Gelegenheiten, Provokationen und Kontrollintensitäten auszeichnen, und den individuellen Neigungen für abweichendes Verhalten, die vor allem durch moralische Überzeugungen und exekutive Funktionen geprägt werden. Das Modell rechnet sowohl mit bedeutenden individuellen Unterschieden in den Wahrnehmungen von und Entscheidungen für Handlungsalternativen als auch mit Unterschieden im kriminogenen Gehalt der räumlichen „Settings“, in denen sich Kriminalität als Ergebnis der Wechselwirkung von Person und Umwelt ereignet. Das Zusammentreffen von Personen und Settings bildet nach der „Situational Action Theory“ die unmittelbaren Ursachen von Kriminalität, während Einflüsse, die zu der Ausprägung von individuellen Verhaltenstendenzen und zur Gestaltung der Settings führen, als „causes of the causes“ eine vorgelagerte und indirekte Kausalwirkung haben und sowohl auf individueller als auch auf verschiedenen räumlichen Ebenen bis hin zu Gesamtgesellschaft angesiedelt sein können. Damit werden in diesem Theoriemodell Elemente des Routine Acitivities Approach und des Rational Choice-Ansatz mit „klassischen" täterorientierten Erklärungsansätzen verbunden, so dass potenziell alle Einflüsse, die einerseits auf die individuellen Verhaltensdispositionen und andererseits auf die Situationen wirken, in das Erklärungsmodell ,eingebaut“ werden können. Die Spannweite der Einflüsse reicht dann nach dem Titel eines einschlägigen Sammelbandes „From Neurons to Neighborhoods" (Shonkoff/Phillips 2000), von der biologischen Ausstattung bis zu den sozialräumlichen Lebenswelten. Diese verschiedenen Ebenen sind in Abbildung 11 als Ringe symbolisiert, die sich um das Individuum legen.

Die Stärke der integrativen Erklärungsansätze und ihre Überlegenheit gegenüber den alten Mehrfaktorenansätzen hängen davon ab, ob es gelingt, die kausalen Beziehungen und Wechselwirkungen zwischen den unterschiedlichen Komponenten des Modells aufzuhellen. Einige punktuelle Ergebnisse dieser noch kaum gelösten Aufgabe sollen im Folgenden angesprochen werden. 


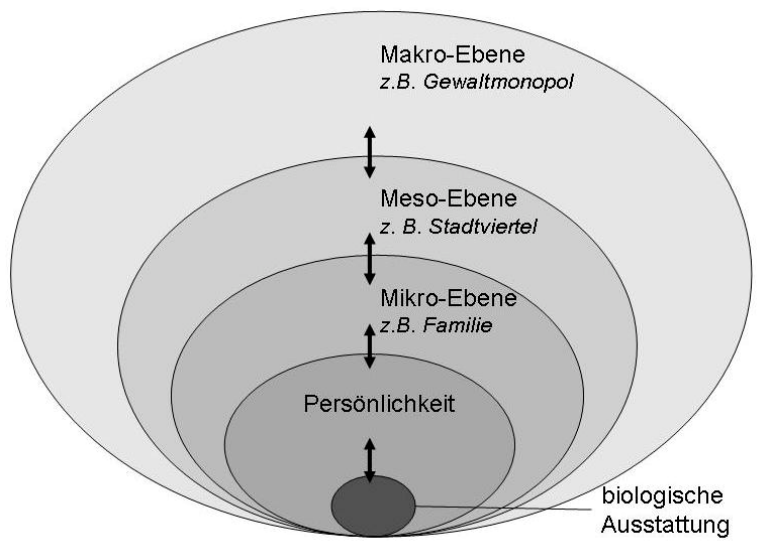

Eine Hypothese der Situational Action Theory lautet, dass positive Anreize durch günstige Tatgelegenheiten und negative Anreize durch Abschreckung ebenso wie die eigene Selbstkontrolle nur das Verhalten derjenigen beeinflussen können, die Normbrüche überhaupt als eine Handlungsalternative in Betracht ziehen, während Menschen mit starken konformen Überzeugungen unabhängig vom Ausmaß ihrer Selbstkontrolle und von den situativen Umständen keine Straftaten begehen werden. Es wird also angenommen, dass die Frage der moralischen Überzeugungen der Frage der situativen Einflüsse einschließlich der individuellen Verhaltenskontrolle in diesen Situationen kausal vorgelagert ist. Dies hat sich in verschiedenen Studien bestätigt, die deutliche Interaktionseffekte zwischen den moralischen Überzeugungen und der Selbstkontrolle oder den Aspekten der Situation gefunden haben (Kroneberg/Heintze/Mehlkop 2010; Schoepfer/Piquero 2006; Svensson/Pauwels 2010; Wikström/Tseloni/Karlis 2008).

Erst wenige Studien haben Wechselwirkungen zwischen Einflussfaktoren auf unterschiedlichen Ebenen untersucht, wie zum Beispiel das Zusammenspiel von individuellen oder familiären Merkmalen auf der Mikroebene mit städtischen Armutskonzentrationen auf der sozialräumlichen Mesoebene. Ein sehr bekanntes Beispiel ist die Studie von Caspi et al. (2002) zur Gen-Umwelt-Interaktion zwischen MAOA-Mangel und frühkindlicher Misshandlung. Ein zweites Beispiel betrifft Mikro-Makro-Interaktionen. Es gibt Hinweise darauf, dass sich ungünstige Bedingungen in Wohnquartieren stärker auf Kinder auswirken, die auch persönliche oder familiäre Risikofaktoren aufweisen, während andere Kinder gegenüber sozialräumlichen Gefährdungen eher unempfänglich (resilient) sind (Beyers et al. 2003; Lahey et al. 2008; Lynam et al. 2000). Eine allgemeine Schlussfolgerung aus diesen Erkenntnissen ist, dass es sich bei den statistischen Effekten einzelner Einflussfaktoren häufig um durchschnittliche Wirkungen handelt, die erheblich stärker oder auch schwächer ausfallen, wenn man sie nicht isoliert betrachtet, sondern in ihren komplexen Wechselwirkungen mit anderen relevanten Merkmalen. Dies ist auch für Präventions- und Interventionsstrategien wichtig. 
Ein weiterer Vorteil integrativer Erklärungsmodelle könnte schließlich darin liegen, dass sie eine Abwägung der relativen kausalen Gewichte von Einflussfaktoren auf unterschiedliche Phänomene und Aspekte von Kriminalität erlauben. Was damit gemeint ist, sei an einem Beispiel erläutert. Anhand von Daten der Pittsburgh Youth Study untersuchten Loeber et al. (2005; Loeber/Pardini 2009), welche Unterschiede hinsichtlich der Belastung mit Risikofaktoren zwischen unauffälligen und gewalttätigen jungen Männern sowie solchen, die sogar ein Tötungsdelikt begangen hatten, bestehen. Zusammengefasst unterscheiden sich unauffällige und gewalttätige junge Männer in einer ganzen Reihe von frühkindlichen Risikofaktoren und Persönlichkeitsmerkmalen. Gewalttätige junge Männer, die Tötungsdelikte begehen, unterscheiden sich von anderen gewalttätigen Männern dagegen kaum mehr im Ausmaß dieser psychologischen Einflussfaktoren, sondern in der Zugehörigkeit zu kriminellen Banden, der Verwicklung in illegale Drogenmärkte und der Verfügbarkeit von Waffen. Damit dominieren für die Erklärung von tödlicher Gewalt klar Merkmale der sozialen Desorganisation großstädtischer Armutsquartiere (ein Aspekt der Mesobzw. Makro-Ebene), während weniger extreme Gewaltneigungen vergleichsweise stärker durch persönliche und familiale Faktoren beeinflusst werden (ein Aspekt der Mikro-Ebene).

\subsection{Empirische Forschungserkenntnisse}

Während ich in den vorangehenden Abschnitten wenige grundlegende Theorieansätze vorgestellt und in diesem Zusammenhang empirische Erkenntnisse, die diese Theorien unterstützen oder in Frage stellen, nur beiläufig erwähnt habe, sollen am Ende dieses Beitrages Ergebnisse der Kriminalitätsforschung zu zwei Themenfeldern in den Mittelpunkt gestellt werden. Sowohl die Beziehungen zwischen Kriminalität und sozialer Ungleichheit als auch die Entwicklung von Kriminalität im Lebenslauf sind zentrale und sehr aktuelle Forschungsfelder, die das sozialwissenschaftliche Verständnis von Delinquenz und Kriminali- tät wesentlich prägen. Es ist bei dieser Auswahl leider unvermeidlich, dass andere, ebenso relevante Themenfelder der Kriminalitätsforschung ignoriert werden. Teils werden diese jedoch in anderen Beiträgen dieses Handbuchs behandelt.

Soziale Ungleichheit, Desorganisation und Kriminalität

Die Verbindung von Sozialer Ungleichheit und Kriminalität ist seit den Anfängen dieser Disziplin einer der „Dauerbrenner“ der Kriminalsoziologie und zieht mit der gegenwärtigen Zunahme von Ungleichheiten wieder mehr Aufmerksamkeit auf sich (vgl. Albrecht 2001; Groenemeyer 2005; Hagan/Peterson 1995; Karstedt 1996; Ludwig-Mayerhofer 2000; Short 1997). Während diese Problematik in den USA schon immer sehr eng mit der Dimension ethnischer Minderheiten verknüpft war, hat sich diese Perspektive in Deutschland erst in dem Maße in den Vordergrund geschoben, in dem es zu einem Einwanderungsland mit multi-ethnischer Bevölkerungsstruktur wurde. Im 19. Jahrhundert prägten die Angst der Mittel- und Oberschichten vor den „Gefährlichen Klassen“, welche sich unter anderem aus den engen Zusammenhängen zwischen Nahrungsmittelpreisen, Hungerrevolten und Eigentumskriminalität speiste (Blasius 1978; Mehlum/Miguel/Ragnar 2006), sowie ethnographische Berichte über großstädtische Elendsquartiere als Orte des Verbrechens wie Henry Mayhews „London Labour and London Poor“ (1861) das Verständnis der Zusammenhänge von Armut und Kriminalität. Willem Bongers Pionierwerk (,Criminality and Economic 
Conditions“, 1916) ebenso wie Robert Mertons Formulierung der Anomietheorie (siehe Abschnitt 5.2) markieren die selbstverständliche Übernahme dieser Verknüpfung in die wissenschaftliche Kriminalsoziologie.

Mit der langen Phase der Wohlstandszunahme nach dem Zweiten Weltkrieg und überraschenden Ergebnissen der Befragungen von Jugendlichen zur selbstberichteten Delinquenz wurde dieser Zusammenhang jedoch zunehmend in Frage gestellt. Charles Tittle (1983; Tittle/Villemez/Smith 1978) bezeichnete die These, dass Menschen aus unteren Sozialschichten „krimineller“ seien als Menschen aus Mittel- und Oberschichten, als „Mythos". Auch in Deutschland zeigten sich bei Dunkelfeldbefragungen von Jugendlichen und Heranwachsenden nur sehr schwache Zusammenhänge zwischen Sozialstatus und Kriminalität (Albrecht 2003; Albrecht/Howe 1992; Boers/Class/Kurz 1994). Mit der methodischen und inhaltlichen Weiterentwicklung der Dunkelfeldbefragungen hat sich das Bild jedoch wieder gewandelt. Jugendliche aus unteren Schichten (und in Deutschland insbesondere in Haupt- und Sonderschulen) begehen tendenziell häufiger und schwerer bewertete Straftaten, was auch ein höheres polizeiliches Registrierungsrisiko mit sich bringt (Albrecht 2003; Farnworth et al. 1994; Hagan 1992; Köllisch 2004). Grundsätzlich kann der Zusammenhang von sozialer Benachteiligung und Kriminalität im Hell- und Dunkelfeld ebenso wie auf der individuellen Mikro- und unterschiedlichen Makroebenen als recht robust angesehen werden.

Analysen der aktuellen Dunkelfeldbefragungen in Deutschland zeigen, dass eine höhe- re Delinquenzbelastung von Jugendlichen aus einigen ethnischen Minderheitengruppen und bei einigen Deliktsformen überwiegend durch deren soziale Marginalisierung erklärt wer- den kann (Enzmann/Brettfeld/Wezels. 2004; Naplava 2005; Rabold/Baier 2007). Das als Migrationsproblem wahrgenommene Thema der so genannten „Ausländerkriminalität“ ist daher in erster Linie, allerdings nicht ausschließlich, ein Problem sozialer Benachteiligungen (G. Albrecht 1972; H.-J. Albrecht 1996; Drewniak 2004; Haug/Rühl/Gostomski 2008; Oberwittler/Lukas 2010).

Zahlreiche Studien haben den Zusammenhang von sozialer Ungleichheit und Kriminalität anhand der Raten von schweren Gewalt- und Tötungsdelikten auf unterschiedlichen räumlichen Aggregierungsebenen, von Stadtvierteln bis Nationen, untersucht. In einer Metaanalyse kommen Pratt/Cullen (2005; vgl. Crutchfield/Wadsworth 2002; Land/McCall/ Cohen 1990; Messner 2002; Pridemore 2002) zu dem Schluss, dass Indikatoren ökonomischer Deprivation alle anderen Indikatoren in der Stärke ihres Zusammenhangs übertreffen. In vielen Studien hat sich bestätigt, dass das Ausmaß von Vermögens- oder Einkommensungleichheit, gemessen z. B. durch den Gini-Koeffizienten, unabhängig von Deprivation einen eigenständigen steigernden Effekt auf Gewaltkriminalität hat (Blau/Blau 1982; Braithwaite/Braithwaite 1980; Hipp 2007; Kovandzic/Vieraitis/Yeisley 1998), wobei allerdings bei strenger methodologischer Kritik die Zusammenhänge offensichtlich komplizierter sind als gedacht (vgl. Albrecht 2001). Gesellschaften mit extremer ökonomischer Ungleichheit wie Südafrika und einige lateinamerikanische Länder gehören auch zu den Ländern mit den höchsten Mordraten.

In einer methodisch besonders sorgfältigen Längsschnittsstudie haben Fajnzylber/Lederman/Loayza (2002) den kausalen Charakter dieses Zusammenhangs nachgewiesen. In einer methodisch vergleichbaren Studie berechneten Raphael/Winter-Ebmer (2001), dass 
ca. 40 Prozent des Rückgangs der Eigentumskriminalität in den USA in den 1980er und 1990er Jahren dem Rückgang der Arbeitslosigkeit zuzuschreiben ist. Auch der rapide Anstieg der Homizid- und Suizidraten in Russland und einigen anderen osteuropäischen Staaten nach 1989 kann in statistischen Modellen mit dem Niedergang des materiellen Lebensstandards, aber auch mit institutionellen Veränderungen und anomischen Entwicklungen im Durkheimschen Sinne erklärt werden (Pridemore/Kim 2007; Pridemore/Chamlin/Cochran 2007; Stamatel 2009). Auf der anderen Seite gibt es auch empirische Belege für einen reziproken Effekt von Kriminalität auf Arbeitslosigkeit und ökonomischen Niedergang sowohl auf der Mikroebene als auch auf der Makroebene (Bussmann/Werle 2004; Greenbaum/Tita 2004; Mehlum/Moene/Ragnar 2005; Morenoff/Sampson 1997).

Das „quasi-natürliche Experiment“ des rapiden politischen und sozialen Wandels in Osteuropa ist ein deutlicher Beleg für den enormen Einfluss, den gesellschaftliche Rahmenbedingungen auf die Kriminalitätsentwicklung haben. Ungeklärt bleiben in den auf der Makro-Ebene angesiedelten Studien jedoch die Mechanismen und vermittelnden Prozesse, durch die diese Rahmenbedingungen auf das individuelle Verhalten einzelner Menschen wirken und die dementsprechend auch nur unter Einbeziehung der Mikro-Ebene identifiziert werden können. Anstelle einer unmittelbaren Beziehung zwischen ökonomischer Deprivation und Kriminalität, für die sich sehr wenige Anhaltspunkte gefunden haben (Boers 1996), werden verschiedene komplexere Wirkungsmechanismen diskutiert. Eine wichtige Dimension ist die gegenüber ökonomischen Deprivationen nicht immune familiäre Stabili- tät und Qualität der Eltern-Kind-Beziehung während der ersten Lebensjahre.

Eine ganz andere Dimension ist die Effektivität und Legitimität des staatlichen Gewaltmonopols. Viele Tötungs- und andere Gewaltdelikte tragen den Charakter von Vergeltungsmaßnahmen in Konflikten, bei denen die Beteiligten keinen Zugang zur staatlichen Sozialkontrolle haben oder zu haben glauben (Kubrin/Weizer 2003a). Kriminelle Handlungen sind in diesen konfliktreichen Beziehungen eine Art von Selbstjustiz, die jedoch nicht erfolgreich zur Eindämmung von Kriminalität beitragen, sondern sie weiter steigern. Nach Donald Blacks (1983; vgl. Cooney 2003, 2006) rechtssoziologischem Erklärungsmodell wird private Vergeltung umso wahrscheinlicher, je größer die Distanz und die Zugangschancen zum staatlichen Gewaltmonopol sind. Trifft dies auf Unterschichtsangehörige ohnehin tendenziell zu, so wird dies in delinquenten Subkulturen und Schwarzmärkten wie z. B. Drogenmärkten, die sich in ökonomisch deprivierten Milieus ausbreiten, und in Situationen der Schwächung staatlicher Ordnung zu einem sehr starken Einflussfaktor für Gewalt (Groenemeyer 2005; Trotha 2002).

Der lebensweltliche Ort, an dem solche und andere Mechanismen greifen können, sind großstädtische Wohnquartiere, in denen aufgrund von Segregationsprozessen sozial benachteiligte Menschen räumlich konzentriert leben, und die stets auch Brennpunkte einiger klassischer Kriminalitätsformen - Mord und Totschlag, Körperverletzung, Raub, Diebstahl sind (Bottoms 2007; Eisner 1997; Morenoff/Sampson 1997; Wikström 1991, 2007b). Die einflussreiche Theorie der „sozialen Desorganisation“ von Shaw/McKay (1969 [1942]) interpretierte diesen Zusammenhang nicht als Ergebnis individueller Delinquenzneigungen, sondern als kollektives Ergebnis der sozialen Funktionsweise lokaler Nachbarschaften, nämlich einer unzureichenden informellen Sozialkontrolle des Verhaltens der Jugendlichen und der subkulturellen „Ansteckung“ der jeweils jüngeren Jugendlichen durch die älteren. 
Diese und ähnliche sozialökologische, auf Stadtviertel fokussierte Theorieansätze haben in den vergangenen Jahren eine starke Renaissance erlebt (Bursik 1988; Kubrin/Weitzer 2003a; Oberwittler 2010; Oberwittler/Rabold/Baier 2011; Sampson/Wilson 1995; Sampson/Morenoff/Gannon-Rowley 2002).

Auch der „Broken Windows“-Ansatz (Wilson/Kelling 1982; vgl. Hess 1999), der Unordnung, Vandalismus und kleine Regelverstöße im öffentlichen Raum als Ausgangspunkt einer Kausalkette sieht, an deren Ende schwere Gewaltkriminalität und ein Niedergang von Stadtvierteln steht, gehört in diesen Rahmen, er konnte sich in der Kriminalsoziologie gegenüber den Nachfolgern des Desorganisationsansatzes aber nicht durchsetzen (Harcourt/Ludwig 2007; Taylor 2001). Zum einen gelang es Robert Sampson (Sampson/Groves 1989; Sampson/Raudenbush/Earls 1997; vgl. Bellair 2000; Browning/Feinberg/Dietz 2004; Friedrichs/Oberwittler 2007) zu zeigen, dass der Zusammenhang zwischen struktureller Benachteiligung und Gewaltbelastung von Stadtvierteln durch den Grad der sozialen Kohäsion und der informellen Sozialkontrolle (zusammengefasst im Konzept der „collective efficacy") kausal vermittelt wird, zum anderen zeigen Studien, dass auch die Kontakte zu delinquenten Gleichaltrigen, wie bereits von Shaw und McKay behauptet, einen wichtigen Effekt auf die Verstärkung delinquenten Verhaltens in den „sozialen Brennpunkten“ ausüben (Harding 2008, 2009; Haynie/Silver/Teasdale 2006; Oberwittler 2004b, 2007).

Die neue statistische Methode der Mehrebenenanalyse spielt in diesem Forschungsfeld eine entscheidende Rolle, da sie die empirische Überprüfung der Hypothese von kollektiven sozialräumlichen Einflüssen auf individuelles Verhalten erst möglich gemacht hat (Raudenbush/Sampson 1999). Mit Mehrebenenmodellen ließ sich anhand von Dunkelfelddaten zeigen, dass sozialstrukturelle Merkmale wie Arbeitslosigkeit der Eltern nicht auf der individuellen Ebene, sondern als kollektives Merkmale des Wohnquartiers (durch die sozialräumliche Segregation von Arbeitslosen) einen signifikanten Einfluss auf Jugenddelinquenz ausüben (Oberwittler 2004a). In den durch eine extreme Segregation und „Ghettoisierung“ (Massey 1990; Wacquant/Wilson 1989; Wilson 1987) geprägten Großstädten der USA sind sozialräumliche Kontexteffekte für einen erheblichen Teil der höheren Kriminalitätsbelastung von afro-amerikanischen Jugendlichen verantwortlich (Bellair/McNulty 2005; Kirk 2008; Peterson/Krivo/Hagan 2006).

Aktuelle Studien untersuchen insbesondere, welche Wechselwirkungen zwischen individuellen und familiären Merkmalen einerseits und Merkmalen der Wohnquartiere andererseits dazu führen, dass Kinder und Jugendliche unterschiedlich auf soziale Kontextbedingungen reagieren, so dass einige stark beeinflussbar und andere resilient sind (Browning/ Leventhal/Brucks-Gunn 2005; Elliott et al. 2006). Die im oben bereits erwähnte „Situatio- nal Action Theory“ stellt einen der bislang elaboriertesten Versuche dar, das Zusammen- spiel individueller Dispositionen mit den sozialräumlichen Kontextbedingungen aufzuklä- ren.

Delinquenz und Kriminalität im Lebenslauf

Viele zentrale Fragestellungen der empirischen Kriminalitätsforschung auf der Mikroebene der Individuen lassen sich am besten oder ausschließlich anhand von Längsschnittdaten und in der Lebenslaufperspektive untersuchen. Dies gilt grundsätzlich für alle Fragen nach Kausalbeziehungen und für Fragen nach den langfristigen Verläufen von Verhaltensformen, 
wie etwa: Welche Bedeutung haben Persönlichkeitsmerkmale und frühkindliche Belastungen für die Neigung zu delinquentem Verhalten im Jugend- und Erwachsenenalter; gibt es typische „kriminelle Karrieren“ und wie werden sie beendet; wie wirken sich strafrechtliche Sanktionen auf das weitere Verhalten aus?

Während für die Analyse von Kausalbeziehungen schon relativ kurze Längsschnitte z. B. mit drei Messzeitpunkten innerhalb der Kindheits- oder Jugendphase hilfreich sind, erfordert die Lebenslaufperspektive extrem lange Beobachtungszeiträume von bis zu mehreren Jahrzehnten, da verlässliche Informationen nur in einem prospektiven Studiendesign mit zeitnahen Messungen gewonnen werden können. Dieses Forschungsdesign wurde bislang nur in sehr wenigen Studien realisiert, wie zum Beispiel in der „Cambridge Study in Delinquent Development“, in der seit 1961 eine Gruppe von 411 damals achtjährigen Jungen aus einem Londoner Arbeiterviertel immer wieder befragt und untersucht werden (Farrington/Coid/West 2009; Piquero/Farrington/Blumstein 2007; West/Farrington 1973). Dieses als „Developmental“ und „Life-Course Criminology“ bezeichnete Forschungsfeld gehört derzeit zu den lebendigsten und produktivsten Gebieten in der empirischen Kriminalitätsforschung und spiegelt dabei einmal mehr die unterschiedlichen Perspektiven der eher auf die Persönlichkeit fokussierten Psychologie/Psychiatrie einerseits und der eher auf soziale Beziehungen und Interaktionen fokussierten Soziologie andererseits wider (für aktuelle Überblicke siehe Boers 2007; Farrington 2003, 2008; Kerner 2005; Sampson/Laub 2005; Schumann 2010; Thomas/Stelly 2008).

Ein zentrales Erkenntnisziel der mehrheitlich dem „Risk Factor Approach“ verpflichteten psychologischen Verlaufsstudien ist die Identifizierung von Merkmalen des Verhaltens und der Lebenssituation in der frühen Kindheit, die eine Prognose späterer Delinquenz und Kriminalität erlauben. Dahinter steht bis heute der Gedanke, die Ursachen von Kriminalität möglichst früh erkennen und kriminelle Karrieren durch rechtzeitige Präventionen und Interventionen, teils bereits während der Schwangerschaft, verhindern zu können (Farrington/ Welsh 2007; Jungmann/Pfeiffer 2010). Wer im Sample der Cambridge-Studie als Kind schlechte Schulleistungen erbrachte, oppositionelles Verhalten zeigte und Eltern hatte, die selbst straffällig geworden waren, schlug später mit einer höheren Wahrscheinlichkeit eine kriminelle Karriere ein (Farrington et al. 2009; vgl. die Kritik an den Befunden dieser Forschungsrichtung bei G. Albrecht 2004: 501 ff.). In der neuseeländischen Dunedin-Studie sagen neuro-kognitive Risikofaktoren, elterliche Erziehungsdefizite und das Aufmerksamkeitsdefizitsyndrom einen frühen Beginn und einen intensiveren Verlauf des delinquenten Verhaltens voraus (Caspi et al. 1994; Moffitt/Caspi 2001). Die statistische Genauigkeit dieser Voraussagen ist jedoch nicht sehr groß und produziert (zum Glück) eine große Anzahl von „falsch Positiven“, die trotz Risikofaktoren keinen delinquenten Entwicklungspfad nehmen (White et al. 1990).

Bereits bei frühen Längsschnittstudien wie der „Philadelphia Birth Cohort“ (Geburtsjahrgang 1945) entwickelten Kriminologen die idealtypische Einteilung von Straffälligen in die große Gruppe relativ unauffälliger Täter, auf die die Merkmale der Ubiquität und Spontanbewährung zutrafen, und einer sehr kleinen Gruppe „chronischer“ Täter, die ihre kriminelle Karriere sehr früh beginnen, über sehr lange Zeiträume hinweg immer wieder auffäl- lig werden und für etwa die Hälfte aller Delikte verantwortlich sind (Wolfgang et al. 1972). Terrie Moffitt (1993, 2006; Moffitt et al. 2002) prägte die Forschung der letzten rund 15 
Jahre nachhaltig mit ihrer Klassifikation der Täter in „life-course-persistent“ (LCP) und „adolescence-limited“ (AL). Während demnach Kriminalität in der AL-Gruppe eine normale, sozialpsychologisch erklärbare Begleiterscheinung des Jugendalters darstellt, wird die LCP-Gruppe als eine Risikogruppe mit psychopathologischen Defiziten angesehen.

Die Versuche, Menschen aufgrund ihrer langfristigen Verlaufsmuster delinquenten Verhaltens in voneinander abgrenzbare Typen einzuteilen, hat durch die Neuentwicklung des speziellen statistischen Verfahrens des ,group-based trajectory modeling“ (auch „,semiparametric modeling“, Nagin 1999, 2005; Nagin/Tremblay 2005; Reinecke 2008) starken Auftrieb erhalten und zu einer Fülle von Analysen mit verschiedenen Längsschnittdaten auf der Basis offizieller oder selbstberichteter Straftaten geführt (Albrecht/Grundies 2009; Blokland/Nagin/Nieuwbeerta 2005; Broidy et al. 2003; Liberman 2008). Das Konzept der „trajectories“ (wörtlich „Bewegungs-, Flugbahn“), also definierter Entwicklungspfade delinquenten Verhaltens im Lebenslauf, legt in Kombination mit der starken Orientierung an Täterpersönlichkeiten eine quasi-deterministische Vorherbestimmung der Verlaufstypen nahe. Damit läuft dieser Ansatz Gefahr, die Eigendynamiken von Lebensläufen in ihren sozialen Bezügen zu vernachlässigen, was von kriminalsoziologischer Seite zu Recht kritisiert wird (Sampson/Laub 2003). Auch innerhalb der Logik dieses Ansatzes haben neue empirische Ergebnisse zunehmend Zweifel insbesondere an Moffitts Typologie genährt. In der noch laufenden Dunedin-Studie beendete ein Teil der Studienteilnehmer in der LCPGruppe seine delinquente Karriere entgegen der Vorhersagen und wechselte in ein eher unauffälliges Verhaltensmuster; auf der anderen Seite bildete sich eine Gruppe von ,adultonset"-Tätern, die trotz einer unauffälligen Kindheit und Jugend erst spät mit einer kriminellen Karriere begannen (Moffit 2006; Moffit et al. 2002). Damit wird auch die Vorhersagekraft eines frühen Delinquenzbeginns (early onset) für die Dauer und Intensität einer kriminellen Karriere in Frage gestellt (Boers 2007).

Einen alternativen, soziologisch orientierten Analyseansatz der Entwicklung von Delinquenz und Kriminalität im Lebenslauf haben John Laub und Robert Sampson ausgearbeitet (Laub/Sampon 2003; Laub/Sampson/Sweeten 2006; Sampson/Laub 1993, 2005, 2009), die die Daten einer alten Längsschnittstudie des Ehepaars Sheldon und Eleanor Glueck reanalysiert und den weiteren Lebensweg der ursprünglichen Studienteilnehmer bis zu deren 70. Lebensjahr weiterverfolgt haben. In Anlehnung an Hirschis Kontrolltheorie sehen Sampson und Laub die im Lebenslauf wechselnde Stärke oder Schwäche sozialer Bindungen als eine wesentliche Ursache der Delinquenzentwicklung an (,Age-graded Theory of Informal Social Control“). Eine vorübergehende Schwächung sozialer Bindungen im Jugendalter erklärt demnach den charakteristischen Gipfel der „Age-Crime“-Kurve, die Übernahme von sozialen Rollen der Erwachsenenwelt wie Berufstätigkeit und Familiengründung ihren charakteristischen Abschwung. Auf der einen Seite können schwerere Formen der Delinquenz und die Reaktionen darauf zu „kumulativen Benachteiligungen“ durch eine nachfolgende weitere Schwächung der sozialen Bindungen und der Ressourcen für eine positive Lebensbewältigung führen, wodurch eine negative Dynamik entsteht. Auf der anderen Seite können plötzliche Ereignisse (life events) wie Schul- und Ortswechsel, Militärdienst oder Heirat „Wendepunkte“ im Lebenslauf sein, die zur Stärkung sozialer Bindungen führen und zu Auslösern eines erfolgreichen Abbruchs krimineller Karrieren werden. Für beinahe alle Täter unabhängig von der Intensität ihres kriminellen Handelns und 
von persönlichen Risikofaktoren sehen Sampson und Laub ein allmähliches Nachlas- sen oder den Abbruch der kriminellen Karriere als den generellen Entwicklungspfad (,lifecourse desister") an. Zu einer sehr ähnlichen, kontrolltheoretisch fundierten Interpretation der Verlaufsformen von Kriminalität im Lebenslauf sind auch die Forscher der in den 1960er Jahren begonnenen „Tübinger Jungtäter-Studie“ gelangt (Mischkowitz 1993; Stelly/ Thomas 2001, 2004; Stelly/Thomas/Kerner 2003).

Der „Karriereabbruch“, der Übergang von delinquentem zu normkonformem Verhalten im Lebenslauf hat in der Kriminalitätsforschung bis vor kurzem sehr viel weniger Aufmerksamkeit erhalten als der Beginn der Delinquenz (Ezell/Cohen 2005; Laub/Sampson 2003; Weitekamp et al. 2000). Durch die Erhebung von Längsschnittdaten jenseits des zweiten oder dritten Lebensjahrzehnts hat sich die empirische Basis für Langzeitbeobachtungen in den letzten Jahren verbessert. Andere Studien haben sich explizit auf die Erwachsenenphase konzentriert und die Entwicklungen von Straffälligen nach ihrer Haftzeit untersucht (Maruna 2001; Stelly/Thomas 2004). Die Prozesse des Beendens von Delinquenz und Kriminalität sind insbesondere statistisch schwerer erklärbar und werden daher häufiger anhand von qualitativen, offenen Interviews untersucht. Dabei wird sehr deutlich, dass die erfolgreiche Überwindung einer kriminellen Karriere - unbeschadet der hilfreichen Rolle der erwähnten Wendepunkte und der sozialen Bindungen - nicht ohne eine kognitive Veränderung und aktive Entscheidung zu erreichen ist, die als Entschlussfähigkeit (Maruna/Farrall 2004; Stelly/Thomas 2004) und zielgerichtete Handlungsmacht (,agency“) (Bottoms 2006; Laub/Sampson 2003; Sampson/Laub 2005) beschrieben wird.

Schließlich bieten Längsschnittstudien auch die Chance, die Auswirkungen von formellen Sanktionen auf das weitere delinquente Verhalten zu untersuchen und dadurch die theoretischen Annahmen des Labeling Approach zu überprüfen. Führen strafrechtliche Sanktionen bei jugendlichen Straftätern durch stigmatisierende Wirkungen zu einer Verstärkung der Delinquenz und zur „sekundären Devianz“ (Lemert 1967)? Diese kriminalpolitisch zentrale Frage wurde von der ätiologisch orientierten Längsschnittforschung nur am Rande untersucht. Bei der Bremer Längsschnittstudie von Haupt- und Sonderschulabsolventen, die über elf Jahre verfolgt wurden, zeigten sich robuste statistische Hinweise auf negative Effekte von strafrechtlichen Sanktionen, d. h. in Richtung auf eine Zunahme von Delinquenz oder eine Verzögerung des Abbruchs (Schumann 2003a, 2003b). Dieser Befund bestätigte sich auch in einem Vergleich der Studie mit einer US-amerikanischen Längsschnittstudie (Ehret 2007; Schumann et al. 2009). Insbesondere scheinen Strafsanktionen die Stabilität eines qualifizierten Beschäftigtenstatus zu erschweren und stellen dadurch ein Risiko für spätere Arbeitslosigkeit dar, während in der Jugendphase häufig ein „Doppelleben“ von erfolgreicher Ausbildung und Delinquenz stattfindet, solange diese nicht zu schweren Sanktionen führt. Interessant ist in diesem Zusammenhang auch der Befund einer Bielefelder Längsschnittstudie, dass sich kurzfristige und langfristige Effekte alternativer Sanktionen unterschedien können; strafende Sanktionen scheinen kurzfristig die Rückfallwahrscheinlichkeit zu reduzieren, bewirken aber längerfristig über Stigmatisierungseffekte das Gegenteil (Albrecht 1995; Crasmöller 1996).

Die Ergebnisse entsprechen insgesamt der oben erwähnten These der „kumulativen Benachteiligung" als Folgewirkung delinquenten Verhaltens: Angestoßen durch straffälliges Verhalten und die Reaktionen darauf verringern sich die für die Bewältigung der Le- 
bensaufgaben notwendigen Ressourcen und Integrationschancen. Auf der anderen Seite ergaben die qualitativen Interviews jedoch auch Hinweise auf die generalpräventive Wirkung drohender Strafsanktionen, die für viele Jugendliche und Heranwachsende eine wichtige Handlungsorientierung darstellen. Die kriminalpolitischen Schlussfolgerungen aus den Ergebnissen der Längsschnittforschung sind damit nicht trivial. Insgesamt zeigt sich auch bei den Längsschnittstudien, dass individuelle Dispositionen und familiäre Faktoren ohne Zweifel bedeutsam sind, dass jedoch soziale Kontexte und soziale Bindungen und Interaktionen - in Wechselwirkungen mit den individuellen Faktoren - einen mit dem Alter vermutlich zunehmenden Einfluss auf delinquentes Verhalten haben.

\section{Literatur}

Aebi, Marcelo F., 2009: Self-Reported Delinquency Surveys in Europe. Paris: CRIMPREV/GERN. Internetquelle: [http://www.gern-cnrs.com/gern/fileadmin/documents/CRIMPREV/WP7/Brochure_2/brochureAebi_WP7_n_ 2_VA.pdf].

Agnew, Robert, 1992: Foundation for a General Strain Theory of Crime and Delinquency. Criminology 30/1: 4787.

Agnew, Robert, 1995: The Contribution of Social-Psychological Strain Theory to the Explanation of Crime and Delinquency. S. 113-137 in: Adler, F./Laufer, W.S. (Hrsg.), The Legacy of Anomy Theory. New Brunswick: Transaction.

Agnew, Robert, 2005: Juvenile Delinquency. Causes and Control [2. Auflage]. Los Angeles: Roxbury.

Agnew, Robert, 2006: Pressured Into Crime. An Overview of General Strain Theory. Los Angeles: Roxbury.

Akers, Ronald L., 1998: Social Learning and Social Structure: A General Theory of Crime and Deviance. Boston: Northeastern University Press.

Akers, Ronald L./Jensen, Gary F., 2003: Social Learning Theory and the Explanation of Crime. A Guide for the New Century. New Brunswick, N.J.: Transaction.

Akers, Ronald L./Jensen, Gary F., 2006: The Empirical Status of Social Learning Theory of Crime and Deviance: The Past, Present, and Future. S. 37-76 in: Cullen, F.T./Wright, J.P./Blevins, K.R. (Hrsg.), Taking Stock. The Status of Criminological Theory (Advances in Criminological Theory, Vol. 15). New Brunswick: Transaction. Albrecht, Günter, 1972: Soziologie der geographischen Mobilität. Stuttgart: Enke.

Albrecht, Günter, 1981: Zwerge auf den Schultern eines Riesen? Neuere Beiträge der Theorien abweichenden Verhaltens und sozialer Kontrolle in der Tradition Emile Durkheims. S. 323-358 in: Alemann, H. von/Thurn, H.P. (Hrsg.), Soziologie in weltbürgerlicher Absicht. Festschrift für René König zum 75. Geburtstag. Opladen: Westdeutscher Verlag.

Albrecht, Günter, 1995: Diversion in Juvenile Justice: Theoretical Expectations and Preliminary Results of a Treatment Outcome Evaluation of Juvenile Offenders. S. 187-226 in: Albrecht, G./Ludwig-Mayerhofer, W. (Hrsg.), Diversion and Informal Social Control. Berlin: de Gruyter.

Albrecht, Günter, 2001: Soziale Ungleichheit, Deprivation und Gewaltkriminalität. S. 195-235 in: Albrecht, G./ Backes, O./Kühnel, W. (Hrsg.), Gewaltkriminalität zwischen Mythos und Realität. Frankfurt/M.: Suhrkamp.

Albrecht, Günter, 2002: Soziologische Erklärungsansätze individueller Gewalt und ihre empirische Bewährung. S. 763-818 in: Heitmeyer, W./Hagan, J. (Hrsg.), Internationales Handbuch der Gewaltforschung. Wiesbaden: Westdeutscher Verlag.

Albrecht, Günter, 2003: Soziallage jugendlicher Straftäter. Ein Vergleich für selbstberichtete Delinquenz im Dunkel- und Hellfeld und die Sanktionspraxis im Kontrollfeld. S. 87-116 in: Raithel, J./Mansel, J. (Hrsg.), Kriminalität und Gewalt im Jugendalter. Hell- und Dunkelfeldbefunde im Vergleich. Weinheim: Juventa.

Albrecht, Günter, 2004: Sinn und Unsinn der Prognose von Gewaltkriminalität. S. 475-524 in: Heitmeyer, W./ Soeffner, H.-G. (Hrsg.), Gewalt. Frankfurt/M.: Suhrkamp.

Albrecht, Günter, 2009: Jugend: Recht und Kriminalität. S. 831-904 in: Krüger, H.-H./Grunert, C. (Hrsg.), Handbuch Kindheits- und Jugendforschung. (2., aktualisierte und erweiterte Auflage). Wiesbaden: VS - Verlag für Sozialwissenschaften.

Albrecht, Günter/Howe, Carl W., 1992: Soziale Schicht und Delinquenz. Verwischte Spuren oder falsche Fährte? Kölner Zeitschrift für Soziologie und Sozialpsychologie 44/4: 697-730. 
Albrecht, Günter/Howe, Carl W./Wolterhoff-Neetix, Jochen, 1988: Neue Ergebnisse zum Dunkelfeld der Jugenddelinquenz: Selbstberichtete Delinquenz von Jugendlichen in zwei westdeutschen Großstädten. S. 661-696 in: Kaiser, G./Kury, H./Albrecht, H.-J. (Hrsg.), Kriminologische Forschung in den 80er Jahren, Bd. 2. Freiburg: Max-Planck-Institut für ausländischen und internationales Strafrecht.

Albrecht, Günter/Ludwig-Mayerhofer, Wolfgang, (Hrsg.) 1995: Diversion and Informal Social Control (Prevention and Intervention in Childhood and Adolescence, Band 17). Berlin: de Gruyter.

Albrecht, Hans-Jörg, 1996: Ethnic Minorities. Crime and Criminal Justice in Germany. S. 31-100 in: Tonry, M. (Hrsg.), Ethnicity, Crime, and Immigration. Comparative and Cross-National Perspectives (Crime and Justice. A Review of Research, Vol. 21). Chicago: University of Chicago Press.

Albrecht, Hans-Jörg, 2004: Öffentliche Meinung, Kriminalpolitik und Kriminalpolitik. S. 491-520 in: Walter, M./ Kania, H./Albrecht, H.-J. (Hrsg.), Alltagsvorstellungen von Kriminalität. Individuelle und gesellschaftliche Bedeutung von Kriminalitätsbildern für die Lebensgestaltung. Münster: Lit.

Albrecht, Hans-Jörg, 2007: Perspektiven kriminologischer Forschung. Der Wandel im Konzept der Sicherheit und neue Aufgabenfelder der Kriminologie. S. 177-202 in: Liebl, K. (Hrsg.), Kriminologie im 21. Jahrhundert. Wiesbaden: VS - Verlag für Sozialwissenschaften.

Albrecht, Hans-Jörg/Grundies, Volker, 2009: Justizielle Registrierungen in Abhängigkeit vom Alter: Befundes aus der Freiburger Kohortenstudie. Monatsschrift für Kriminologie und Strafrechtsreform 92/2-3: 326-343.

Anderson, Elijah, 1999: Code of the Street. Decency, Violence, and the Moral Life of the Inner City. New York: W.W. Norton.

Anhut, Reimund/Heitmeyer, Wilhelm, 2009: Desintegration, Anerkennungsbilanzen und die Rolle sozialer Vergleichsprozesse für unterschiedliche Verarbeitungsmuster. S. 212-236 in: Preyer, G. (Hrsg.), Neuer Mensch und kollektive Identität in der Kommunikationsgesellschaft. Wiesbaden: VS - Verlag für Sozialwissenschaften.

Arnold, Harald, 1986: Kriminelle Viktimisierung und ihre Korrelate. Ergebnisse international vergleichender Opferbefragungen. Zeitschrift für die gesamt Strafrechtswissenschaft 98: 1014-1058.

Aromaa, Kauko, 1991: Notes on the Victimization Experience. Interviews with Victims of Violence. S. 589-609 in: Kaiser, G./Kury, H./Albrecht, H.-J./Arnold, H. (Hrsg.), Victims and Criminal Justice. Freiburg i. Br: MaxPlaack-Institut für ausländisches und internationales Strafrecht.

Asendorpf, Jens B., 2008: Genetische Grundlagen der Sozialisation. S. 70-82 in: Hurrelmann, K./Grundmann, M./ Walper, S. (Hrsg.), Handbuch Sozialisationsforschung [7.Auflage]. Weinheim: Beltz.

Atran, Scott, 2003: The Genesis of Suicide Terrorism. Science 299: 1534-1539.

Baier, Dirk, 2008: Entwicklung der Jugenddelinquenz und ausgewählter Bedingungsfaktoren seit 1998 in den Städten Hannover, München, Stuttgart und Schwäbisch Gmünd. Hannover: KFN.

Baier, Dirk/Pfeiffer, Christian/Windzio, Michael/Rabold, Susann 2006: Schülerbefragung 2005: Gewalterfahrungen, Schulabsentismus und Medienkonsum von Kindern und Jugendlichen (Abschlussbericht über eine repräsentative Befragung von Schülerinnen und Schülern der 4. und 9. Jahrgangsstufe). Hannover: KFN.

Baier, Dirk/Pfeiffer, Christian/Simonson, Julia/Rabold, Susann, 2009: Jugendliche in Deutschland als Opfer und Täter von Gewalt. Erster Forschungsbericht zum gemeinsamen Forschungsprojekt des Bundesministeriums des Innern und des KFN (KFN Forschungsbericht 107). Hannover: KFN.

Baier, Dirk/Windzio, Michael, 2008: Zur Entwicklung der Jugendgewalt seit 1998 in den Städten München, Stuttgart, Hannover und Schwäbisch Gmünd. S. 4560-4575 in: Rehberg, K.-S. (Hrsg.), Die Natur der Gesellschaft. Verhandlungen des 33. Kongresses der Deutschen Gesellschaft für Soziologie in Kassel 2006. Frankfurt/M.: Campus.

Bandura, Albert, 1973: Aggression: A Social Learning Analysis. New York: Holt.

Baumann, Ulrich, 2000: Das Verbrechensopfer in Kriminalitätsdarstellungen der Presse. Eine empirische Untersuchung der Printmedien. Freiburg: edition iuscrim.

Baumgartner, Thomas/Heinrichs, Markus/Vonlanthen, Aline/Fischbacher, Urs/Fehr, Ernst, 2008: Oxytocin Shapes the Neural Circuitry of Trust and Trust Adaptation in Humans. Neuron 58: 639-650.

Beaver, Kevin M./Wright, John P./Delisi, Matt, 2007: Self-Control as an Executive Function: Reformulating Gottfredson and Hirschi's Parental Socialization Thesis. Criminal Justice and Behavior 34/10: 1345-1361.

Beaver, Kevin M./Wright, John P./Delisi, Matt/Vaughn, Michael G., 2008: Genetic Influences on the Stability of Low Self-Control. Results from a Longitudinal Sample of Twins. Journal of Criminal Justice 36: 478-485.

Bechara, Antoine/Damasio, Hannah/Damasio, Antonio R., 2000: Emotion, Decision Making, and the Orbitofrontal Cortex. Cerebral Cortex 10/3: 295-307.

Becker, Gary S., 1968: Crime and Punishment. An Economic Approach. Journal of Political Economy 76: 169217.

Becker, Howard S., 1967: Whose Side are We On? Social Problems 14/3: 239-247. 
Becker, Howard S., 1981: Außenseiter. Zur Soziologie abweichenden Verhaltens. Frankfurt/M.: Fischer [am. org. 1963: Outsiders. Studies of the Sociology of Deviance. New York: Free Press].

Becker, Melanie, 2007: Kriminalpolitische Paradigmen und alltagsweltliche Deutungsmuster zu Kriminalität und Kriminalitätsbekämpfung. S. 46-70 in: Hess, H./Ostermeier, L./Paul, B. (Hrsg.), Kontrollkulturen. Texte zur Kriminalpolitik im Anschluss an David Garland (Kriminologisches Journal 9. Beiheft). Weinheim: Juventa.

Becker, Peter/Wetzell, Richard F., 2006: Criminals and Their Scientists. The History of Criminology in International Perspective. Cambridge: Cambridge University Press.

Beckett, Katherine, 1997: Making Crime Pay. Law and Order in Contemporary American Politics. Oxford: Oxford University Press.

Bellair, Paul E., 2000: Informal Surveillance and Street Crime. A Complex Relationship. Criminology 38/1: 137169.

Bellair, Paul E./McNulty, Thomas L., 2005: Beyond the Bell Curve. Community Disadvantage and the Explanation of Black-White Differences in Adolescent Violence. Criminology 43/4: 1135-1168.

Bendixen, Mons/Endresen, Inger M./Olweus, Dan, 2006: Joining and Leaving Gangs. Selection and Facilitation Effects on Self-Reported Antisocial Behaviour in Early Adolescence. European Journal of Criminology 3/1: $85-114$.

Bernburg, Jón G./Krohn, Marvin D./Rivera, Craig J., 2006: Official Labeling, Criminal Embeddedness, and Subsequent Delinquency A Longitudinal Test of Labeling Theory. Journal of Research in Crime and Delinquency 43/1: 67-88.

Beyers, Jennifer M./Bates, John E./Pettit, Gregory S./Dodge, Kenneth A., 2003: Neighborhood Structure, Parenting Processes, and the Development of Youths Externalizing Behaviors. A Multilevel Analysis. American Journal of Community Psychology 31/1-2: 35-53.

Bieneck, Steffen/Oswald, Margit E./Hupfeld-Heinemann, Jörg, 2009: Social Psychology of Punishment of Crime. Chicester: John Wiley \& Sons.

Black, Donald J., 1983: Crime as Social Control. American Sociological Review 48/1: 34-45.

Blasius, Dirk, 1978: Kriminalität und Alltag. Zur Konfliktgeschichte des Alltagslebens im 19. Jahrhundert. Göttingen: Vandenhoeck \& Ruprecht.

Blau, Judith R./Blau, Peter M., 1982: The Cost of Inequality. Metropolitan Structure and Violent Crime. American Sociological Review 47/1: 114-129.

Blokland, Arjan A. J./Nagin, Daniel S./Nieuwbeerta, Paul, 2005: Life Span Offending Trajectories of a Dutch Conviction Cohort. Criminology 43/4: 919-954.

Boers, Klaus, 1993: Kriminalitätsfurcht: Ein Beitrag zum Verständnis eines sozialen Problems. Monatsschrift für Kriminologie und Strafrechtsreform 76/1: 65-82.

Boers, Klaus, 1996: Sozialer Umbruch und Kriminalität in Deutschland. Monatsschrift für Kriminologie und Strafrechtsreform 79/5: 314-337.

Boers, Klaus, 2001: Kriminalprävention und Kriminalpolitik mit der Kriminalitätsfurcht? Neue Kriminalpolitik 2: $10-15$.

Boers, Klaus, 2002: Furcht vor Gewaltkriminalität. S. 1399-1422 in: Heitmeyer, W./Hagan, J. (Hrsg.), Internationales Handbuch der Gewaltforschung. Wiesbaden: Westdeutscher Verlag.

Boers, Klaus, 2007: Hauptlinien der kriminologischen Längsschnittforschung. S. 5-40 in: Boers, K./Reinecke, J. (Hrsg.), Delinquenz im Jugendalter: Erkenntnisse einer Münsteraner Längsschnittstudie. Münster: Waxman.

Boers, Klaus, 2008: Kontinuität und Abbruch persistenter Delinquenzverläufe. S. 340-376 in: Deutsche Vereinigung für Jugendgerichte und Jugendgerichtshilfen e.V. (DVJJ) (Hrsg.), Fördern Fordern Fallenlassen. Aktuelle Entwicklungen im Umgang mit Jugenddelinquenz. Mönchengladbach: Forum Verlag Godesberg.

Boers, Klaus/Class, Michael/Kurz, Peter, 1994: Self-Reported Delinquency in Germany after the Refunification. S. 343-353 in: Junger-Tas, J./Terlouw, G.-J./Klein, M.W. (Hrsg.), Delinquent Behavior Among Young People in the Western World. First Results of the International Self-Report Delinquency Study. Amsterdam: RDC Ministery of Justice.

Boers, Klaus/Kurz, Peter, 1997: Kriminalitätseinstellungen, soziale Milieus und sozialer Umbruch. S. 187-253 in: Boers, K./Gutsche, G./Sessar, K. (Hrsg.), Sozialer Umbruch und Kriminalität in Deutschland. Opladen: Westdeutscher Verlag.

Boers, Klaus/Kurz, Peter, 2001: Kriminalitätsfurcht ohne Ende? S. 123-144 in: Albrecht, G./Backes, O./Kühnel, W. (Hrsg.), Gewaltkriminalität zwischen Mythos und Realität. Frankfurt/M.: Suhrkamp.

Boers, Klaus/Reinecke, Jost, 2007: Delinquenz im Jugendalter. Erkenntnisse einer Münsteraner Längsschnittstudie. Münster: Waxmann

Boers, Klaus/Theile, Hans/Karliczek, Kari-Maria, 2004: Wirtschaft und Strafrecht - Wer reguliert wen? S. 469493 in: Oberwittler, D./Karstedt, S. (Hrsg.), Soziologie der Kriminalität (Sonderheft 43 der Kölner Zeitschrift für Soziologie und Sozialpsychologie). Wiesbaden: VS - Verlag für Sozialwissenschaft. 
Boers, Klaus/Walburg, Christian/Reinecke, Jost, 2006: Jugendkriminalität - Keine Zunahme im Dunkelfeld, kaum Unterschiede zwischen Einheimischen und Migranten. Befunde aus Duisburger und Münsteraner Längsschnittstudien. Monatsschrift für Kriminologie und Strafrechtsreform 89/2: 63-87.

Böllinger, Lorenz/Jasch, Michael/Krasmann, Susanne, 2010: Gefährliche Menschenbilder. Biowissenschaften, Gesellschaft und Kriminalität. Baden-Baden: Nomos.

Bonger, Willem A., 1916: Criminality and Economic Conditions. London: Heinemann [org. 1906: Dissertation, Universität Amsterdam].

Bottoms, Anthony E., 2006: Desistance, Social Bonds, and Human Agency. A Theoretical Exploration. S. 243-290 in: Wikström, P.-O.H./Sampson, R.J. (Hrsg.), The Explanation of Crime. Context, Mechanisms and Development. Cambridge: Cambridge University Press.

Bottoms, Anthony E., 2007: Place, Space, Crime and Disorder. S. 528-574 in: Maguire, M./Morgan, R./Reiner, R. (Hrsg.), The Oxford Handbook of Criminology [4. Auflage]. Oxford: Oxford University Press.

Boudon, Raymond, 1998: Social Mechanisms without Black Boxes. S. 172-203 in: Hedström, P./Swedberg, R. (Hrsg.), Social Mechanisms. An Analytical Approach to Social Theory. Cambridge: Cambridge University Press.

Braga, Anthony A., 2005: Hot Spots Policing and Crime Prevention. A Systematic Review of Randomized Controlled Trials. Journal of Experimental Criminology 1: 317-342.

Braithwaite, John, 1989: Crime, Shame, and Reintegration. Cambridge.: Cambridge University Press

Braithwaite, John, 2004: Transkontinentale Migration von Strafgefangenen: Das Beispiel Australien. Ein Beitrag zur Soziologie der Strafe. S. 413-440 in: Oberwittler, D./Karstedt, S. (Hrsg.), Soziologie der Kriminalität (Sonderheft 43 der Kölner Zeitschrift für Soziologie und Sozialpsychologie). Wiesbaden: VS - Verlag für Sozialwissenschaften.

Braithwaite, John/Braithwaite, Valerie, 1980: The Effect of Income Inequality and Social Democracy on Homicide. British Journal of Criminology 20: 45-53.

Brodeur, Jean-Paul/Ouellet, Genevieve, 2004: What is a Crime? A Secular Answer. S. 1-33 in: Law Commission of Canada (Hrsg.), What Is a Crime? Defining Criminal Conduct in Contemporary Society. Vancouver: UBC.

Broidy, Lisa M./Tremblay, Richard E./Brame, Bobby/Fergusson, David/Dodge, Kenneth A./Horwood, John L./ Loeber, Rolf/Laird, Robert/Lynam, Donald R./Moffitt, Terrie E./Nagin, Daniel S./Bates, John E./Pettit, Gregory S./Vitaro, Frank, 2003: Developmental Trajectories of Childhood Disruptive Behaviors and Adolescent Delinquency: A Six Site, Cross National Study. Developmental Psychology 39: 222-245.

Brown, Stephen E./Esbensen, Finn-Age/Geis, Gilbert, 2001: Criminology. Explaining Crime and its Context. (4. Auflage). Cincinnati, Ohio: Anderson.

Browning, Christopher R./Feinberg, Seth L./Dietz, Robert D., 2004: The Paradox of Social Organization: Networks, Collective Efficacy, and Violent Crime in Urban Neighborhoods. Social Forces 83/2: 503-534.

Browning, Christopher R./Leventhal, Tama/Brooks-Gunn, Jeanne, 2005: Sexual Initiation in Early Adolescence: The Nexus of Parental and Community Control. American Sociological Review 70: 758-778.

Brüchert, Oliver, 2005: Autoritäres Programm in aufklärerischer Absicht. Wie Journalisten Kriminalität sehen. Münster: Westfälisches Dampfboot.

Bundeskriminalamt (BKA), 2007: Polizeiliche Kriminalstatistik 2007 Bundesrepublik Deutschland. Berichtsjahr 2006. Wiesbaden: BKA. Internetquelle: [http://www.bka.de/pks/pks2006/download/pks-jb 2006 bka.pdf ].

Bundesministerium des Inneren (BMI)/Bundesministerium der Justiz (BMJ), 2001: Erster Periodischer Sicherheitsbericht. Berlin: BMI/BMJ. Internetquelle: [http://www.bmj.de/files///147/Periodischer\%20Sicherheitsbericht\%201.pdf].

Bundesministerium des Inneren (BMI)/Bundesministerium für Justiz (BMJ), 2006: Zweiter Periodischer Sicherheitsbericht. Berlin: BMI/BMJ. Internetquelle: [http://www.bmj.bund.de/files/-/1485/2.\%20Periodischer\%20 Sicherheitsbericht\%20Langfassung.pdf].

Burgess, Robert L./Akers, Ronald L., 1966: A Differential Association-Reinforcement Theory of Criminal Behavior. Social Problems 14/2: 128-147.

Bursik, Robert J., Jr., 1988: Social Disorganization and Theories of Crime and Delinquency: Problems and Prospects. Criminology 26/4: 519-552.

Bussmann, Kai-Dieter, 2005: Report über die Auswirkungen des Gesetzes zur Ächtung der Gewalt in der Erziehung. Berlin: Bundesministerium der Justiz.

Bussmann, Kai-Dieter/PricewaterhouseCoopers, 2007: Wirtschaftskriminalität 2007. Sicherheitslage der deutschen Wirtschaft. Halle an der Saale. Internetquelle: [http://www.pwc.de/fileserver/RepositoryItem/studie wikri_2007.pdf?itemId=3169192].

Bussmann, Kai-Dieter/Werle, Markus, 2004: Kriminalität - Standortfaktor für betriebliche Entscheidungen? Neue Kriminalpolitik 16/3: 90-95.

Caplan, Bryan, 2006: Terrorism: The Relevance of the Rational Choice Model. Public Choice 128: 91-107. 
Carr, Patrick/Napolitano, Laura/Keating, Jessica, 2007: We Never Call the Cops and Here is Why. A Qualitative Examination of Legal Cynicism in three Philadelphia Neighborhoods. Criminology 45/2: 445-480.

Caspi, Avshalom/McClay, Joseph/Moffitt, Terrie E./Mill, Jonathan/Craig, Ian W./Taylor, Alan/Poulton, Richie, 2002: Role of Genotype in the Cycle of Violence in Maltreated Children. Science 297: 851-854.

Caspi, Avshalom/Moffitt, Terrie E./Silva, Phil A./Stouthamer-Loeber, Magda/Krueger, Robert F./Schmutte, Pamela S., 1994: Are some People Crime-Prone? Replications of the Personality-Crime Relationship across Countries, Genders, Races, and Methods. Criminology 32/2: 163-195.

Catalano, Shannon M., 2006: Criminal Victimzation, 2005 (Bureau of Justice Statistics Bulletin). Washington, DC: U. S. Department of Justice.

Cavadino, Michael/Dignan, James, 2006: Penal Policy and Political Economy. Criminology \& Criminal Justice 6/4: 435-456.

Cloward, Richard A./Ohlin, Lloyd E., 1960: Delinquency and Opportunity. A Theory of Delinquent Gangs. New York: Free Press.

Cohen, Albert K., 1961: Kriminelle Jugend. Reinbek: Rowohlt [am. org. 1955: Delinquent Boys. The Culture of the Gang. Glencoe: Free Press].

Cohen, Lawrence E./Felson, Marcus, 1979: Social Change and Crime Rate Trends: A Routine Activity Approach. American Sociological Review 44: 588-608.

Cohen, Stanley, 1972: Folk Devils and Moral Panics. The Creation of the Mods and Rockers. London: MacGibbon $\&$ Kee.

Cohen, Stanley, 1988: The Object of Criminology: Reflections on the New Criminilization. S. 235-276 in: Cohen, S., Against Criminology. New Brunswick, N.J, Transaction.

Coleman, James S., 1986: Social Theory, Social Research, and a Theory of Action. American Journal of Sociology 91: 1309-1335.

Coleman, James S., 1990: Foundations of Social Theory. Cambridge, Mass.: Harvard University Press.

Cooney, Mark, 2003: Privatization of Violence. Criminology 41/4: 1377-1407.

Cooney, Mark, 2006: The Criminological Potential of Pure Sociology. Crime, Law and Social Change 46: 51-63.

Cornish, Derek B./Clarke, Ronald V., 1986: The Reasoning Criminal. Rational Choice Perspectives on Offending. New York: Springer.

Crasmöller, Bernhard, 1996: Wirkungen strafrechtlicher Sozialkontrolle jugendlicher Kriminalität. Eine empirische Analyse der spezialpräventiven Effekte staatsanwaltlicher Diversion. Pfaffenweiler: Centaurus.

Crawford, Adam (Hrsg.), 2002: Crime and Insecurity. The Governance of Safety in Europe. Cullompton: Willan.

Cremer-Schäfer, Helga/Steinert, Heinz, 1998: Straflust und Repression. Zur Kritik der populistischen Kriminologie. Münster: Westfälisches Dampfboot.

Crick, Nicki R./Dodge, Kenneth A., 1994: A Review and Reformulation of Social Information-Processing Mechanisms in Children's Social Adjustment. Psychological Bulletin 115/1: 74-101.

Crutchfield, Robert D./Wadsworth, Tim, 2002: Armut und Gewalt. S. 83-103 in: Heitmeyer, W./Hagan, J. (Hrsg.), Internationales Handbuch der Gewaltforschung. Wiesbaden: Westdeutscher Verlag.

Cullen, Francis T./Clark, Gregory A./Cullen, John B./Mathers, Richard A., 1985: Attribution, Salience, and Attitudes toward Criminal Sanctioning. Criminal Justice and Behavior 12/3: 305-331.

Cullen, Francis T./Wright, John P./Blevins, Kristie R., 2006: Taking Stock. The Status of Criminological Theory. Roxbury: Transaction.

de Haan, Willem/Vos, Jaco, 2004: Widersprüchliche Gefühle: Rationalität und Emotionalität im Entscheidungsverhalten von jugendlichen Straftätern. S. 316-336 in: Oberwittler, D./Karstedt, S. (Hrsg.), Soziologie der Kriminalität (Sonderheft 43 der Kölner Zeitschrift für Soziologie und Sozialpsychologie). Wiesbaden: VS - Verlag für Sozialwissenschaften.

de Quervain, Dominique J.F./Fischbacher, Urs/Treyer, Valerie/Schellhammer, Melanie/Schnyder, Ulrich/Buck, Alfred/Fehr, Ernst, 2004: The Neural Basis of Altruistic Punishment. Science 305: 1254-1258.

Depue, Richard A., 2009: Genetic, Environmental, and Epigenetic Factors in the Development of Personality Disturbance. Development and Psychopathology 21: 1031-1063.

Diekmann, Andreas, 2008: Soziologie und Ökonomie: Der Beitrag experimenteller Wirtschaftsforschung zur Sozialtheorie. Kölner Zeitschrift für Soziologie und Sozialpsychologie 60/3: 528-550.

Diewald, Martin, 2010: Zur Bedeutung genetischer Variation für die soziologische Ungleichheitsforschung. Zeitschrift für Soziologie 39/1: 4-21.

Dinkes, Rachel/Catildi, Emily F./Lin-Kelly, Wendy/Snyder, Thomas D., 2007: Indicators of School Crime and Safety: 2007. Washington, DC: U.S. Department of Education.

Dittmann, Jörg, 2005: Entwicklung der Kriminalitätseinstellungen in Deutschland. Eine Zeitreihenanalyse anhand allgemeiner Bevölkerungsumfragen (DIW Berlin, Discussion Papers 468). Berlin: DIW. Internetquelle: [http://www.econstor.eu/bitstream/10419/18319/1/dp468.pdf]. 
Dittmann, Jörg, 2009: Unsicherheit in Zeiten gesellschaftlicher Transformation. Zur Entwicklung und Dynamik von Sorgen in der Bevölkerung in Deutschland (SOEP Papers on Multidisciplinary Panel Data Research 243). Berlin: DIW. Internetquelle: [http://www.diw.de/documents/publikationen/73/diw_01.c.344459.de/diw_sp02 43.pdf].

Ditton, Jason/Farrall, Stephen (Hrsg.), 2000: Fear of Crime. Ashgate: Aldershot.

Dodge, Kenneth A./Pettit, Gregory S., 2003: A Biopsychosocial Model of the Development of Chronic Conduct Problems in Adolescence. Developmental Psychology 39/2: 349-371.

Dollinger, Bernd/Raithel, Jürgen, 2006: Einführung in die Theorien abweichenden Verhaltens. Weinheim: Beltz.

Dowler, Kenneth, 2003: Media Consumption and Public Attitudes toward Crime and Justice. The Relationship between Fear of Crime, Punitive Attitudes, and Perceived Police Effectiveness. Journal of Criminal Justice and Popular Culture 10/2: 109-126.

Drewniak, Regine, 2004: „Ausländerkriminalität“ zwischen „kriminologischen Binsenweisheiten“ und „,ideologischem Minenfeld“. Zeitschrift für Jugendkriminalrecht und Jugendhilfe 4: 372-378.

Dülmer, Hermann, 2007: Experimental Plans in Factorial Surveys: Random or Quota Design? Sociological Methods Research 35: 382-409.

Durkheim, Emile, 1982: Die Regeln der soziologischen Methode. Frankfurt/M.: Suhrkamp [fr. org. 1895: Les régles de la méthode sociologique. Paris: Alcan].

Durkheim, Emile, 1977: Über soziale Arbeitsteilung. Studie über die Organisation höherer Gesellschaften. Frankfurt/M.: Suhrkamp [fr. org. 1893: De la division du travail social: étude sur l'organisation des sociétés supérieurs. Paris: Alcan].

Durkheim, Emile, 1992: Two Laws of Penal Evolution. S. 21-49 in: Gane, M. (Hrsg.), The Radical Sociology of Durkheim and Mauss. London: Routledge [fr. org. 1901: Deux lois de l'évolution pénale. Année Sociologique 4: 65-95].

Egg, Rudolf (Hrsg.), 2000: Was tun mit den Sexualstraftätern? Rationalität und Irrationalität der Reaktionen in Politik, Justiz und Gesellschaft. Köln: Wienand.

Ehret, Beate, 2007: Strafen oder Erziehen? Eine komparative Längsschnittstudie zu den Auswirkungen strafrechtlicher Verfolgung von Jugenddelinquenz in Bremen, Deutschland und Denver, Col., USA. Münster: Lit.

Eifler, Stefanie, 2002: Kriminalsoziologie. Bielefeld: transcript.

Eifler, Stefanie, 2007: Evaluating the Validity of Self-Reported Deviant Behavior Using Vignette Analyses. Quality \& Quantity 41: 303-318.

Eifler, Stefanie, 2009: Kriminalität im Alltag. Eine handlungstheoretische Analyse von Gelegenheiten. Wiesbaden: VS - Verlag für Sozialwissenschaften.

Eisner, Manuel, 1997: Das Ende der zivilisierten Stadt? Die Auswirkungen von Modernisierung und urbaner Krise auf Gewaltdelinquenz. Frankfurt/M.: Campus.

Eisner, Manuel, 2002: Crime, Problem Drinking, and Drug Use. Patterns of Problem Behavior in Cross-National Perspective. Annales of the American Academy of Political and Social Science 580/March: 201-225.

Eisner, Manuel/Manzoni, Patrik/Ribeaud, Denis, 2000: Gewalterfahrungen von Jugendlichen. Opfererfahrungen und selbstberichtete Gewalt bei Schülerinnen und Schülern im Kanton Zürich. Aarau: Sauerländer.

Eisner, Manuel/Ribeaud, Denis, 2003: Erklärung von Jugendgewalt - eine Übersicht über zentrale Forschungsbefunde. S. 182-206 in: Raithel, J./Mansel, J. (Hrsg.), Kriminalität und Gewalt im Jugendalter. Weinheim: Juventa.

Elliott, Delbert S./Menard, Scott/Rankin, Bruce H./Wilson, William J./Huizinga, David, 2006: Good Kids from Bad Neighborhoods. Successful Development in Social Context. Cambridge: Cambridge University Press.

Ellis, Lee/Cooper, Jonathan A./Walsh, Anthony, 2008: Criminologists" Opinions about Causes and Theories of Crime and Delinquency. A Follow-Up. The Criminologist 33/3: 23-26.

Entorf, Horst, 2008: Wirkung und Effizienz von Strafrecht: Was geht - bei jungen Gewaltätern? (ZEW Discussion Papers 08-056). German Working Papers in Law and Economics 16. Zentrum für Europäische Wirtschaftsforschung. Internetquelle: [http://www.bepress.com/cgi/viewcontent.cgi?article=1246\&context=gwp].

Enzmann, Dirk/Brettfeld, Katrin/Wetzels, Peter, 2004: Männlichkeitsnormen und die Kultur der Ehre. Empirische Prüfung eines theoretischen Modells zur Erklärung erhöhter Delinquenzraten jugendlicher Migranten. S. 264287 in: Oberwittler, D./Karstedt, S. (Hrsg.), Soziologie der Kriminalität (Sonderheft 43 der Kölner Zeitschrift für Soziologie und Sozialpsychologie). Wiesbaden: VS - Verlag für Sozialwissenschaften.

Erikson, Kai T., 1978: Die widerspenstigen Puritaner. Zur Soziologie abweichenden Verhaltens. Stuttgart: KlettCotta. [am. org. 1966: Wayward Puritans. A Study in the Sociology of Deviance. New York: Wiley].

Espelage, Dorothy L./Holt, Melissa K./Henkel, Rachael R., 2003: Examination of Peer Group Contextual Effects on Aggression During Early Adolescence. Child Development 74/1: 205-220.

Esping-Andersen, Gøsta, 1990: The Three Worlds of Welfare Capitalism. Cambridge: Polity Press. 
Esser, Hartmut, 2005: Rationalität und Bindung. Das Modell der Frame Selection und die Erklärung des normativen Handels (Arbeitspaper des Sonderforschungsbereichs 504). Universität Mannheim.

Ezell, Michael E./Cohen, Lawrence E., 2005: Desisting from Crime. Continuity and Change in Long-Term Crime Patterns of Serious Chronic Offenders. Oxford: Oxford University Press.

Fajnzylber, Pablo/Lederman, Daniel/Loayza, Norman, 2002: Inequality and Violent Crime. Journal of Law and Economics XLV: 1-40.

Farnworth, Margaret/Thornberry, Terence P./Krohn, Marvin D./Lizotte, Alan J., 1994: Measurement in the Study of Class and Delinquency. Integrating Theory and Research. Journal of Research in Crime and Delinquency 31/1: 32-61.

Farrall, Stephen, 2004: Revisiting Crime Surveys: Emotional Response without Emotions? Or - Look Back in Anger. International Journal of Social Research Methodology 7/2: 151-171.

Farrall, Stephen/Bannister, Jon/Ditton, Jason/Gilchrist, Elizabeth, 1997: Questioning the Measurement of the "Fear of Crime". Findings from a Major Methodological Study. British Journal of Criminology 37/4: 658-679.

Farrall, Stephen/Jackson, Jonathan/Gray, Emily, 2009: Social Order and the Fear of Crime in Contemporary Times. Oxford: Oxford University Press.

Farrell, Graham/Pease, Ken, 2007: Sting in the Tail of the British Crime Survey. Multiple Victimisation. S. 33-52 in: Hough, M./Maxfield, M. (Hrsg.), Surveying Crime in the 21 st Century: Commemorating the 25th Anniversary of the British Crime Survey Monsy. New York: Criminal Justice Press.

Farrington, David P., 2003: Developmental and Life-Course Criminology. Key Theoretical and Empirical Issues. Criminology 41/2: 221-256.

Farrington, David P., 2008: Building Developmental and Life-Course Theories of Offending. S. 335-364 in: Cullen, F.T./Wright, J.P./Blevins, K.R. (Hrsg.), Taking Stock. The Status of Criminological Theory (Advances in Criminological Theory, Vol. 15). New Brunswick: Transaction.

Farrington, David P./Coid, Jeremy/West, Donald J., 2009: The Development of Offending from Age 8 to Age 50. Recent Results from the Cambridge Study in Delinquent Development. Monatsschrift für Kriminologie und Strafrechtsreform 92/2/3: 160-173.

Farrington, David P./Welsh, Brandon C., 2007: Saving Children from a Life of Crime. Early Risk Factors and Effective Interventions. Oxford: Oxford University Press.

Fehr, Ernst/Gächter, Simon, 2002: Altruistic Punishment in Humans. Nature 415: 137-140.

Fehr, Ernst/Gintis, Herbert, 2007: Human Motivation and Social Cooperation. Experimental and Analytical Foundations. Annual Review of Sociology 33: 43-64.

Felson, Marcus, 1994: Crime and Everyday Life. Insights and Implications for Society. Thousand Oaks, Cal.: Pine Forge Press.

Felson, Marcus/Clarke, Ronald V., 1998: Opportunity Males the Thief. Practical Theory for Crime Prevention. London: Home Office, Policing and Reducing Crime Unit.

Fetchenhauer, Detlef, 1998: Versicherungsbetrug. Eine theoretische und empirische Analyse betrügerischen Verhaltens gegenüber einem anonymen Geschädigten. Baden-Baden: Nomos.

Freese, Jeremy, 2008: Genetics and the Social Science Explanation of Individual Outcomes. American Journal of Sociology 114/Supplement: S1-S35.

Frehsee, Detlev, 1991: Zur Abweichung der Angepassten. Kriminologisches Journal 23: 25-45.

Frehsee, Detlev/Löschper, Gabi/Smaus, Gerlinda (Hrsg.), 1997: Konstruktion der Wirklichkeit durch Kriminalität und Strafe. Baden-Baden: Nomos.

Friedrichs, Jürgen/Oberwittler, Dietrich, 2007: Soziales Kapital in Wohngebieten. S. 450-486 in: Franzen, A./Freitag, M. (Hrsg.), Sozialkapital. Wiesbaden: VS - Verlag für Sozialwissenschaften.

Frommel, Monika, 2005: Die Reform des Sexualstrafrechts: strafrechtliche Konsequenzen einer veränderten Sexualmoral, symbolische Politik oder beides? S. 525-544 in: Winter, G. (Hrsg.), Zivilrecht im Sozialstaat. Festschrift für Professor Peter Derleder. Baden-Baden: Nomos.

Fuchs, Marek/Lamnek, Siegfried/Luedtke, Jens/Baur, Nina, 2008: Gewalt an Schulen 1994 - 1999 - 2004. (2. Auflage). Wiesbaden: VS - Verlag für Sozialwissenschaften.

Gabriel, Ute, 1998: Furcht und Strafe. Kriminalitätsfurcht, Kontrollüberzeugungen und Strafforderungen in Abhängigkeit von der Erfahrung krimineller Viktimisierung. Baden-Baden: Nomos.

Gabriel, Ute/Greve, Werner, 2003: The Psychology of Fear of Crime. Conceptual and Methodological Perspectives. British Journal of Criminology 43/3: 600-614.

Gabriel, Ute/Oswald, Margit E., 2007: Psychological Analyses of Punishment. S. 1252-1254 in: Clark, D.S (Hrsg.), Encyclopedia of Law and Society. American and Global perspectives, Band 3. London: Sage.

Galvan, Adriana/Hare, Todd A./Parra, Cindy E., 2006: Earlier Development of the Accumbens Relative to Orbitofrontal Cortex Might Underlie Risk-Taking Behavior in Adolescents. Journal of Neuroscience 26: 6885-6892.

Garland, David, 1985: Punishment and Welfare. A History of Penal Strategies. Aldershot: Ashgate. 
Garland, David, 1990a: Framework of Inquiry in the Sociology of Punishment. British Journal of Sociology 41/1: $1-15$.

Garland, David, 1990b: Punishment and Modern Society. A Study in Social Theory. Oxford: Clarendon Press.

Garland, David, 1991: Sociological Perspectives on Punishment. S. 115-165 in: Tonry, M. (Hrsg.), Crime and Justice. An Annual Review of Research, Vol. 14. Chicago: Chicago University Press.

Garland, David, 1999: The Commonplace and the Catastrophic. Interpretations of Crime in Late Modernity. Theoretical Criminology 3/3: 353-364.

Garland, David, 2001: The Culture of Control. Crime and Social Order in Contemporary Society. Oxford: Oxford University Press [deutsche Übersetzung 2008: Kultur der Kontrolle. Verbrechensbekämpfung und soziale Ordnung in der Gegenwart. Frankfurt/M.: Campus].

Garland, David, 2004: Die Kultur der „High Crime Societies“. Voraussetzungen einer neuen Politik von „Law and Order“. S. 36-68 in: Oberwittler, D./Karstedt, S. (Hrsg.), Soziologie der Kriminalität (Sonderheft 43 der Kölner Zeitschrift für Soziologie und Sozialpsychologie). Wiesbaden: VS - Verlag für Sozialwissenschaften.

Garland, David, 2007: High Crime Societies and Cultures of Control. S. 231-249 in: Hess, H./Ostermeier, L./Paul, B. (Hrsg.), Kontrollkulturen. Texte zur Kriminalpolitik im Anschluss an David Garland (Kriminologisches Journal, 9. Beiheft). Weinheim: Juventa.

Garofalo, Raffaele, 1891: Criminologia. Studio sul delitto e sulla teoria della repressione. Turin: Bocca.

Gershoff, Elizabeth T., 2002: Corporal Punishment by Parents and Associated Child Behaviors and Experiences: A Meta-Analytic and Theoretical Review. Psychological Bulletin 128/4: 539-579.

Gianotti, Lorena R. R./Knoch, Daria/Faber, Pascal L., 2009: Tonic Activity Level in the Right Prefrontal Cortex Predicts Individuals"e Risk Taking. Psychological Science 20/1: 33-38.

Giedd, Jay N., 2008: The Teen Brain: Insights from Neuroimaging. Journal of Adolescent Health 42: 335-343.

Gintis, Herbert/Bowles, Samuel/Boyd, Robert/Fehr, Ernst, 2005: Moral Sentiments and Material Interests. Origins, Evidence, and Consequences. S. 3-39 in: Gintis, H./Bowles, S./Boyd, R./Fehr, E. (Hrsg.), Moral Sentiments and Material Interests. The Foundations of Cooperation in Economic Life. Cambridge, Mass.: MIT Press.

Girling, Evi/Loader, Ian/Sparks, Richard, 2000: Crime and Social Change in Middle England. Questions of Order in an English Town. London: Routledge.

Görgen, Thomas, 2004a: "As if I just didn"t exist". Elder Abuse and Neglect in Nursing Homes. S. 71-89 in: Wahidin, A./Cain, M.E. (Hrsg.), Ageing, Crime and Society. Cullompton: Willan.

Görgen, Thomas, 2004b: A Multimethod Study on Elder Abuse and Neglect in Nursing Homes. Journal of Adult Protection 6/3: 15-25.

Gottfredson, Michael R., 2006: The Empirical Status of Control Theory in Criminology. S. 77-100 in: Cullen, F.T./Wright, J.P./Blevins, K.R. (Hrsg.), Taking Stock. The Status of Criminological Theory (Advances in Criminological Theory, Vol. 15). Roxbury: Transaction.

Gottfredson, Michael R./Hirschi, Travis, 1990: A General Theory of Crime. Stanford, Cal.: Stanford University Press.

Grasmick, Harold G./Bursik, Robert J., 1990: Conscience, Significant Others, and Rational Choice: Extending the Deterrence Model. Law Society Review 24/3: 837-861.

Grasmick, Harold G./Tittle, Charles R./Bursik, Robert J./Arnkler, Bruce J., 1993: Testing the Core Empirical Implications of Gottfredson and Hirschi's General Theory of Crime. Journal of Research in Crime and Deliquency 30/1: 5-29.

Green, David A., 2007: Comparing Penal Cultures. Child-on-Child Homicide in England and Norway. S. 591-643 in: Tonry, M. (Hrsg.), Crime, Punishment, and Politics in a Comparative Perspective (Crime and Justice. A Review of Research, Vol. 36). Chicago: Chicago University Press.

Green, David A., 2008: When Children Kill Children: Penal Populism and Political Culture. Oxford: Oxford University Press.

Greenbaum, Robert T./Tita, George E., 2004: The Impact of Violence Surges on Neighbourhood Business Activity. Urban Studies 41/13: 2495-2514.

Greve, Werner, 1998: Fear of Crime among the Elderly. Foresight, not Fright. International Review of Victimology $5: 277-309$.

Greve, Werner/Hosser, Daniela/Wetzels, Peter, 1996: Bedrohung durch Kriminalität im Alter. Kriminalitätsfurcht älterer Menschen als Brennpunkt einer Gerontokriminologie. Baden-Baden: Nomos.

Groenemeyer, Axel, 2001: Von der Sünde zum Risiko? - Bilder abweichenden Verhaltens und die Politik sozialer Probleme am Ende des Rehabilitationsideals. S. 146-182 in: Groenemeyer, A. (Hrsg.), Soziale Probleme Konstruktivistische Kontroversen und gesellschaftliche Herausforderungen (Soziale Probleme 12/1-2). Herbolzheim: Centaurus.

Groenemeyer, Axel, 2003: Soziale Probleme und politische Diskurse - Konstruktionen von Kriminalpolitik in sozialen Kontexten. (Schriftenreihe Soziale Probleme, Gesundheit und Sozialpolitik. Materialien und For- 
schungsberichte 3). Bielefeld: Fakultät für Soziologie. Internetquelle: [http://www.uni-bielefeld.de/sozprob/ schriftenreihe.htm].

Groenemeyer, Axel, 2005: Ordnungen der Exklusion - Ordnungen der Gewalt. Eine Frage der Ehre? Überlegungen zur Analyse des Zusammenhangs von Exklusion und Gewalt. Soziale Probleme 15/2: 5-40.

Guo, Guang/Roettger, Michael E./Cai, Tianji, 2008: The Integration of Genetic Propensities into Social-Control Models of Delinquency and Violence among Male Youths. American Sociological Review 73/4: 543-568.

Gürerk, Özgür/Irlenbusch, Bernd/Rockenbach, Bettina, 2006: The Competitive Advantage of Sanctioning Institutions. Science 312: 108-111.

Häfele, Joachim/Lüdemann, Christian, 2006: ,Incivilities,, und Kriminalitätsfurcht im urbanen Raum. Kriminologisches Journal 38/4: 273-291.

Hagan, John, 1992: The Poverty of a Classless Criminiology (The American Society of Criminology 1991 Presidential Address). Criminology 30/1: 1-19.

Hagan, John/Hefler, Gerd/Classen, Gabriele/Merkens, Hans, 1998: Subterranean Sources of Subcultural Delinquency Beyond the American Dream. Criminology 36: 309-339.

Hagan, John/Peterson, Ruth D. (Hrsg.), 1995: Criminal and Inequality. Standorf, Cal.: Stanford University Press.

Hale, Chris, 1996: Fear of Crime: A Review on the Literature. International Review of Victimology 4: 79-150.

Hanak, Gerhard, 1987: Ethnographie der Konfliktverarbeitung. Kriminalsoziologische Bibliografie 14/56-57: 934 .

Hanak, Gerhard/Stehr, Johannes/Steinert, Heinz, 1989: Ärgernisse und Lebenskatastrophen. Über den alltäglichen Umgang mit Kriminalität. Bielefeld: Ajz.

Harcourt, Bernard E./Ludwig, Jens, 2007: Reefer Madness: Broken Windows Policing and Misdemeanor Marijuana Arrests In New York City, 1989-2000. Criminology \& Public Policy 6/1: 165-182.

Harding, David J., 2008: Neighborhood Violence and Adolescent Friendships. The International Journal of Conflict and Violence 2/1: 28-55.

Harding, David J., 2009: Violence, Older Peers, and the Socialization of Adolescent Boys in Disadvantaged Neighborhoods. American Sociological Review 74: 445-464.

Hassemer, Winfried/Reemtsma, Jan P., 2002: Verbrechensopfer. Gesetz und Gerechtigkeit. München: Beck.

Haug, Sonja/Rühl, Stefan/Gostomski, Christian B. von, 2008: Migranten als Täter und Opfer von Gewalt und Kriminalität. Bewährungshilfe 55/3: 211-227.

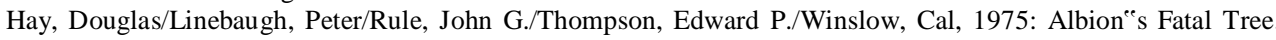
Crime and Society in Eighteenth-Century England. New York: Pantheon Books.

Haynie, Dana L., 2001: Delinquent Peers Revisited: Does Network Structure Matter? American Journal of Sociology 106/4: 1013-1057.

Haynie, Dana L., 2002: Friendship Networks and Delinquency: The Relative Nature of Peer Delinquency. Journal of Quantitative Criminology 18/2: 99-134.

Haynie, Dana L./Osgood, D. W., 2005: Reconsidering Peers and Delinquency: How Do Peers Matter? Social Forces 84/2: 1109-1130.

Haynie, Dana L./Silver, Eric/Teasdale, Brent, 2006: Neighborhood Characteristics, Peer Networks, and Adolescent Violence. Journal of Quantitative Criminology 22: 147-169.

Heinz, Wolfgang, 1990: Kriminalstatistik. Wiesbaden: BKA.

Heinz, Wolfgang, 2008a: Das strafrechtliche Sanktionensystem und die Sanktionierungspraxis in Deutschland 1882-2006. Konstanzer Inventar Sanktionsforschung 2008 (Stand: Berichtsjahr 2006, Version 1/2008). Internetquelle: [http://www.uni-konstanz.de/rtf/kis/Sanktionierungspraxis-in-Deutschland-Stand-2006.pdf].

Heinz, Wolfgang, 2008b: Stellungnahme zur aktuellen Diskussion um eine Verschärfung des Jugendstrafrechts. Zeitschrift für Jugendkriminalrecht und Jugendhilfe 1: 87-96.

Heinz, Wolfgang/Spieß, Gerhard/Schnell, Rainer/Kreuter, Frauke, 1998: Opferbefragungen 1997. Bericht für das Bundesministerium der Justiz. Konstanz: Universität Konstanz.

Heitmeyer, Wilhelm, 1994: Das Desintegrations-Theorem. Ein Erklärungsansatz zu fremdenfeindlich motivierter, rechtsextremistischer Gewalt und zur Lähmung gesellschaftlicher Institutionen. S. 29-72 in: Heitmeyer, W. (Hrsg.), Das Gewalt-Dilemma. Gesellschaftliche Reaktionen auf fremdenfeindliche Gewalt und Rechtsextremismus. Frankfurt/M.: Suhrkamp.

Heitmeyer, Wilhelm (Hrsg.), 1997: Was treibt die Gesellschaft auseinander? Bundesrepublik Deutschland: Auf dem Weg von der Konsensgesellschaft zur Konfliktgesellschaft, Band 1. Frankfurt/M: Suhrkamp.

Heitmeyer, Wilhelm/Collmann, Birgit/Conrads, Jutta/Matuschek.Ingo/Kraul, Dietmar/Kühnel, Wolfgang/Möller, Renate/Ulbrich-Hermann, Matthias, 1996: Gewalt. Schattenseiten der Individualisierung bei Jugendlichen aus unterschiedlichen Milieus. Weinheim: Juventa. [2. Aufl].

Hess, Henner, 1999: Fixing Broken Windows and Bringing Down Crime. Die New Yorker Polizeistrategie der neunziger Jahre. Kritische Justiz 32: 32-57. 
Hess, Henner/Ostermeier, Lars/Paul, Bettina (Hrsg.), 2007: Kontrollkulturen. Texte zur Kriminalpolitik in Anschluss an David Garland (Kriminologisches Journal, 9. Beiheft). Weinheim: Juventa.

Hess, Henner/Scheerer, Sebastian, 1997: Was ist Kriminalität? Skizze einer konstruktivistischen Kriminalitatstheorie. Kriminologisches Journal 29/2: 83-155.

Hess, Henner/Scheerer, Sebastian, 2004: Theorie der Kriminalität. S. 69-92 in: Oberwittler, D./Karstedt, S. (Hrsg.), Soziologie der Kriminalität (Sonderheft 43 der Kölner Zeitschrift für Soziologie und Sozialpsychologie). Wiesbaden: VS - Verlag für Sozialwissenschaften.

Hewstone, Miles/Augoustinos, Martha, 2005: Soziale Attributionen und soziale Repräsentationen. S. 78-99 in: Flick, U. (Hrsg.), Psychologie des Sozialen. Repräsentationen in Wissen und Sprache. Reinbek bei Hamburg: Rowohlt.

Hindelang, Michael J./Gottfredson, Michael R./Garofalo, James, 1978: Victims of Personal Crime: An Empirical Foundation For a Theory of Personal Victimization. Cambridge, Mass.: Ballinger.

Hipp, John R., 2007: Income Inequality, Race, and Place. Does the Distribution of Race and Class within Neighborhoods Affect Crime Rates. Criminology 45/3: 665-697.

Hirschi, Travis, 1969: Causes of Delinquency. Berkeley, Cal.: University of California Press.

Hirtenlehner, Helmut, 2006: Kriminalitätsfurcht. Ausdruck generalisierter Ängste und schwindender Gewissheiten? Kölner Zeitschrift für Soziologie und Sozialpsychologie 58/2: 307-331.

Hirtenlehner, Helmut, 2007: Disorder, Social Anxieties and Fear of Crime. Exploring the Relationship between Incivilities and Fear of Crime with a Special Focus on Generalized Insecurities. S. 127-158 in: Kury, H. (Hrsg.), Fear of Crime - Punitivity. New Developments in Theory and Research. Bochum: Universitätsverlag Brockmeyer.

Hoffmann, Johann G., 1847: Über die Unzulässigkeit eines Schlusses auf Sitten-Verfall aus der Vermehrung der gerichtlichen Untersuchungen gegen jugendliche Verbrecher (Vortrag in der Königlichen Preußischen Akademie der Wissenschaften am 11. Januar 1838). S. 169-185 in: Hoffmann, J.G. (Hrsg.), Nachlass kleiner Schriften staatswirthschaftlichen Inhalts. Berlin: G. Reimer.

Holtappels, Heinz G./Heitmeyer, Wilhelm/Melzer, Wolfgang/Tillmann, Klaus-Jürgen, 1997: Forschung über Gewalt an Schulen: Erscheinungsformen und Ursachen, Konzepte und Prävention. Weinheim, München: Juventa.

Hood, Kathryn E., 2005: Toward an Integrative Account of the Development of Aggressive Behavior. S. 225-252 in: Stoff, D.M./Susman, E.J. (Hrsg.), Developmental Psychobiology of Aggression. Cambridge: Cambridge University Press.

Hood, Roger, 2001: Capital Punishment. A Global Perspective. Punishment \& Society 3: 331-354.

Hummelsheim, Dina/Hirtenlehner, Helmut/Jackson, Jonathan/Oberwittler, Dietrich, 2010: Social Insecurities and Fear of Crime. A Cross-National Study on the Impact of Welfare State Policies on Crime-Related Anxieties. European Sociological Review (online veröffentlicht am 3. März 2010).

Hurrelmann, Klaus/Lösel, Friedrich, 1990: Health Hazards in Adolescence. Berlin: de Gruyter.

Innes, Martin, 2004: Signal Crimes and Signal Disorders. Notes on Deviance as Communicative Action. British Journal of Sociology 55/3: 335-355.

Jackson, Jonathan, 2004a: An Analysis of a Construct and Debate. S. 35-64 in: Albrecht, H.-J./Serassis, T./Kania, H. (Hrsg.), Images of Crime II: Representations of Crime and the Criminal in Politics, Society, the Media, and the Arts. Freiburg i. Br.: edition iuscrim.

Jackson, Jonathan, 2004b: Experience and Expression. Social and Cultural Significance in the Fear of Crime. British Journal of Criminology 44/6: 946-966.

Jackson, Jonathan, 2006: Introducing Fear of Crime to Risk Research. Risk Analysis 26: 253-264.

Jacobs, David/Kleban, Richard, 2003: Political Institutions, Minorities, and Punishment. A Pooled Cross-National Analysis of Imprisonment Rates. Social Forces 80/2: 725-755

Jessor, Richard (Hrsg.), 1998: New Perspectives on Adolescent Risk Behavior. Cambridge: Cambridge University Press.

Jones, T. A./Scull, Andrew T., 1992: Durkheimes "Two Laws of Penal Evolution". An Introduction. S. 13-20 in: Gane, M. (Hrsg.), The Radical Sociology of Durkheim and Mauss. London: Routledge.

Jost, John T./Glaser, Jack/Kruglanski, Arie W./Sulloway, Frank J., 2003: Political Conservatism as Motivated Social Cognition. Psychological Bulletin 129: 339-375

Junger, Marianne/Tremblay, Richard E., 1999: Self-Control, Accidents, and Crime. Criminal Justice and Behavior 26/4: 485-501.

Junger-Tas, Josine/Haen Marshall, Ineke, 1999: The Self-Report Methodology in Crime Research. S. 291-367 in: Tonry, M. (Hrsg.), Crime and Justice. A Review of Research, Vol. 25. Chicago, London: University of Chicago Press. 
Junger-Tas, Josine/Marshall, Ineke H./Enzmann, Dirk/Killias, Martin/Steketee, Majone/Gruazczynska, Beate, 2010: Juvenile Dlinquency and Beyond. Results of the Second International Self-Report Delinquency Study. Dordrecht: Springer.

Jungmann, Tanja/Pfeiffer, Christian, 2010: Zur Notwendigkeit von Prävention aus kriminologischer Perspektive. In: Kißgen, R./Heinen, N. (Hrsg.), Frühe Risiken und frühe Hilfen: Grundlagen, Diagnostik, Prävention. Stuttgart: Klett-Cotta (im Erscheinen).

Kaiser, Günther/Kury, Helmut/Albrecht, Hans-Jörg (Hrsg.), 1991: Victims and Criminal Justice. Legal Protection, Restitution and Support. Freiburg i. Br.: edition iuscrim.

Karstedt, Susanne, 1996: Soziale Ungleichheit und Kriminalität - Zurück in die Zukunft? S. 45-72 in: Bussmann, K.D./Kreissl, R. (Hrsg.), Kritische Kriminologie in der Diskussion. Opladen: Westdeutscher Verlag.

Karstedt, Susanne, 2000: Standortprobleme: Kriminalsoziologie in Deutschland. Soziologische Revue 23: 141151.

Karstedt, Susanne, 2002: Emotions and Criminal Justice. Theoretical Criminology 6/3: 299-317.

Karstedt, Susanne, 2004: „Das tun doch alle“. Anmerkungen zur moralischen Ökonomie moderner Marktgesellschaften. S. 331-351 in: Walter, M./Kania, H./Albrecht, H.-J. (Hrsg.), Alltagsvorstellungen von Kriminalität. Münster: Lit.

Karstedt, Susanne, 2006: Emotions, Crime and Justice: Exploring Durkheimian Themes. S. 203-223 in: Deflem, M. (Hrsg.), Sociological Theory and Criminological Research: Views from Europe and the United States. Amsterdam: Elsevier.

Karstedt, Susanne, 2007a: Die Vernunft der Gefühle. Emotionen, Kriminalität und Strafrecht. S. 25-45 in: Hess, H./Ostermeier, L./Paul, B. (Hrsg.), Kontrollkulturen. Texte zur Kriminalpolitik in Anschluss an David Garland (Kriminologisches Journal, 9. Beiheft). Weinheim: Juventa.

Karstedt, Susanne, 2007b: Explorations into the Sociology of Criminal Justice and Punishment. Leaving the Modernist Project Behind. History of the Human Sciences 20/2: 51-70.

Karstedt, Susanne/Farrall, Stephen, 2006: The Moral Economy of Everyday Life. Markets, Consumers and Citizens. British Journal of Criminology 46: 1011-1036.

Karstedt, Susanne/Greve, Werner, 1996: Die Vernunft des Verbrechens. S. 171-210 in: Bussmann, K.D./Kreissl, R. (Hrsg.), Kritische Kriminologie in der Diskussion. Opladen: Westdeutscher Verlag.

Karstedt, Susanne/Oberwittler, Dietrich, 2004: Neue Perspektiven der Kriminalsoziologie. S. 7-35 in: Oberwittler, D./Karstedt, S. (Hrsg.), Soziologie der Kriminalität (Sonderheft 43 der Kölner Zeitschrift für Soziologie und Sozialpsychologie). Wiesbaden: VS - Verlag für Sozialwissenschaften.

Katz, Jack, 1988: Seductions of Crime. Moral and Sensual Attractions of Doing Evil. New York: Basic Books.

Kennedy, Leslie W./Forde, David R., 1990: Routine Activities and Crime. An Analysis of Victimization in Canada. Criminology 28: 137-151.

Kerner, Hans-Jürgen, 2005: Book Review Essay: The Complex Dynamics of the Onset, the Development, and the Termination of a Criminal Career. Lessons on Repeat Offenders to be Drawn from Recent Longitudinal Studies in Criminology. The Annals of the American Academy of Political and Social Science 602: 159-279.

Kilchling, Michael, 1995: Opferinteressen und Strafverfolgung. Freiburg: edition iuscrim.

Killias, Martin, 2002: Grundriss der Kriminologie. Eine europäische Perspektive. Bern: Stämpfli.

Killias, Martin/Haymoz, Sandrine/Lamon, Philippe, 2007: Swiss Crime Survey. Die Kriminalität in der Schweiz im Lichte der Opferbefragungen von 1985 bis 2005. Bern: Stämpfli.

Kim-Cohen, Julia/Moffitt, Terrie E./Caspi, Avshalom/Taylor, Alan, 2004: Genetic and Environmental Processes in Young Children"s Resilience and Vulnerability to Socioeconomic Deprivation. Child Development 75/3: 651-668.

King, Peter, 2000: Crime, Justice and Discretion, Law and Social Relations in England 1740-1820. Oxford: Oxford University Press.

King, Peter, 2004: The Summary Courts and Social Relations in Eighteenth-Century England. Past \& Present 183 : $125-172$.

Kirk, David S., 2008: The Neighborhood Context of Racial and Ethnic Disparities in Arrest. Demography 45/1: 55-77.

Kivivuori, Janne, 2007: Delinquent Behaviour in Nordic Capital Cities. Helsinki. Scandinavian Research Council for Criminology/National Research Institute of Legal Policy, Finland.

Klein, Malcom W./Maxson, Cheryl L., 2006: Street Gang Patterns and Policies. Oxford: Oxford University Press.

Klein, Malcolm W./Weerman, Frank M./Thornberry, Terence P., 2006: Street Gang Violence in Europe. European Journal of Criminology 3/4: 413-437.

Klimke, Daniela, 2008: Wach- und Schließgesellschaft Deutschland. Sicherheitsmentalitäten in der Spätmoderne. Wiesbaden: VS - Verlag für Sozialwissenschaften. 
Klimke, Daniela/Lautmann, Rüdiger, 2006: Die neoliberale Ethik und der Geist des Sexualstrafrechts. Zeitschrift für Sexualforschung 19: 97-117.

Knoch, Daria/Fehr, Ernst, 2007: Resisting the Power of Temptations. The Right Prefrontal Cortex and Self-Control. Annals of the New York Academy of Sciences 1104/1: 123-134.

Köllisch, Tilman, 2004: Vom Dunkelfeld ins Hellfeld. Anzeigeverhalten und Polizeikontakte bei Jugenddelinquenz. Freiburg: Universitätsbibliothek Freiburg: Freiburger Dokumentenserver. Internetquelle: [http://freidok.ub.uni-freiburg.de/volltexte/1686/].

Köllisch, Tilman, 2007: Risikomanagement und selektive Punitivität als Kriminalpolitik von unten: Zum Zeitverlauf des Hell-Dunkelfeldverhältnisses der Jugendgewalt in Deutschland zwischen 1986 und 2003. Kriminologisches Journal 39/4: 243-259.

Köllisch, Tilman, 2009: Vom Dunkelfeld ins Hellfeld: Zur Theorie und Empirie selektiver Kriminalisierung Jugendlicher bei Körperverletzungsdelikten. Monatsschrift für Kriminologie und Strafrechtsreform 92/1: 28-53.

Köllisch, Tilman/Oberwittler, Dietrich, 2004: Sozialer Wandel des Risikomanagements bei Kindern und Jugendlichen. Eine Replikationsstudie zur langfristigen Zunahme des Anzeigeverhaltens. Zeitschrift für Soziologie der Erziehung und Sozialisation 24/1: 49-72.

Kovandzic, Tomislav V./Vieraitis, Lynne M./Yeisley, Mark R., 1998: The Structural Covariates of Urban Homicide. Reassessing the Impact of Income Inequalitiy and Poverty in the Post-Reagan Era. Criminology 36/3: 569600 .

Kreissl, Reinhard/Steinert, Heinz, 2008: Für einen gesellschaftstheoretisch aufgeklärten Materialismus. Soziologische Lehren für die Neurophysiologie - und umgekehrt. Kriminologisches Journal 40: 269-283.

Kroneberg, Clemens, 2005: Die Definition der Situation und die variable Rationalität der Akteure. Ein allgemeines Modell des Handelns. Zeitschrift für Soziologie 34/5: 344-363.

Kroneberg, Clemens/Heintze, Isolde/Mehlkop, Guido, 2008: On Shoplifting and Tax Fraud: An Action-Theoretic Analysis of Crime (Sonderforschungsbereich 504, Working Paper 08-16). Mannheim: Universität Mannheim.

Kroneberg, Clemens/Heintze, Isolde/Mehlkop, Guido, 2010: The Interplay of Moral Norms and Instrumental Incentives in Crime Causation. Criminology 48/1: 259-294.

Kubrin, Charis E./Stucky, Thomas D./Krohn, Marvin D., 2009: Researching Theories of Crime and Deviance. Oxford: Oxford University Press.

Kubrin, Charis E./Weitzer, Ronald, 2003: New Directions In Social Disorganization Theory. Journal of Crime and Delinquency 40/4: 374-402.

Kubrin, Charis E./Weitzer, Ronald, 2003: Retaliatory Homicide: Concentrated Disadvantage and Neighborhood Culture. Social Problems 50/2: 157-180.

Kunczik, Michael/Zipfel, Astrid, 2006: Gewalt und Medien [5. Auflage]. Ein Studienhandbuch. Köln: Böhlau.

Kunz, Karl-Ludwig, 2004: Kriminologie. Eine Grundlegung. Bern; Stuttgart; Wien: Haupt Verlag.

Kury, Helmut (Hrsg.), 2007: Fear of Crime - Punitivity. New Developments in Theory and Research. Bochum: Universitätsverlag Brockmeyer.

Kury, Helmut/Brandenstein, Martin/Obergfell-Fuchs, Joachim, 2009: Dimensions of Punitiveness in Germany. European Journal on Criminal Policy and Research 15: 63-81.

Kury, Helmut/Ferdinand, Theodore, 1999: Public Opinion and Punitivity. International Journal of Law and Psychiatry 22/3-4: 373-392.

Kury, Helmut/Ferdinand, Theodore, 2008: International Perspectives on Punitivity. Bochum: Universitätsverlag Brockmeyer.

Kury, Helmut/Kania, Harald/Obergfell-Fuchs, Joachim, 2004: Worüber sprechen wir, wenn wir über Punitivität sprechen? Versuch einer konzeptionellen und empirischen Begriffsbestimmung. S. 51-88 in: Lautmann, R./ Klimke, D./Sack, F. (Hrsg.), Punitivität (Kriminologisches Journal, 8. Beiheft). Weinheim: Juventa.

Kury, Helmut/Obergfell-Fuchs, Joachim/Würger, Michael, 2000: Gemeinde und Kriminalität. Eine Untersuchung in Ost- und Westdeutschland. Freiburg i. Br.: edition iuscrim.

Kürzinger, Josef, 1978: Private Strafanzeige und polizeiliche Reaktion. Berlin: Duncker \& Humblot.

Lacey, Nicola, 2008: The Prisoners Dilemma. Political Economy and Punishment in Contemporary Democracies. Cambridge: Cambridge University Press.

LaGrange, Randy L./Ferraro, K. F./Supancic, M., 1992: Perceived Risk and Fear of Crime: Role of Social and Physical Incivilities. Journal of Research in Crime and Delinquency 29/3: 311-334.

Lahey, Benjamin B./Van Hulle, Carol A./Onofrio, Brian M./Lee Rodgers, Joseph/Waldman, Irwin D., 2008: Is Parental Knowledge of their Adolescent Offspringe"s Whereabouts and Peer Associations Spuriously Associated with Offspring Delinquency? Journal of Abnormal Child Psychology 36: 807-823.

Lamnek, Siegfried, 2007: Theorien abweichenden Verhaltens I: „Klassische“ Ansätze [8. Auflage]. Paderborn: Wilhelm Fink. 
Lamnek, Siegfried, 2008: Theorien abweichenden Verhaltens II: „Moderne“ Ansätze [3. Auflage]. Paderborn: Wilhelm Fink.

Land, Kenneth C./McCall, Patricia L./Cohen, Lawrence E., 1990: Structural Covariates of Homicide Rates. Are there any Invariances across Time and Social Space? American Journal of Sociology 95/4: 922-963.

Laub, John H./Sampson, Robert J., 2001: Understanding Desistance from Crime. S. 1-69 in: Tonry, M. (Hrsg.), Crime and Justice. A Review of Research, Vol. 28. Chicago: University of Chicago Press.

Laub, John H./Sampson, Robert J., 2003: Shared Beginnings, Divergent Lives. Delinquent Boys to Age 70. Cambridge, Mass.: Harvard University Press.

Laub, John H./Sampson, Robert J./Sweeten, Gary A., 2006: Assessing Sampson and Laub"s Life-Course Theory of Crime. S. 313-333 in: Cullen, F.T./Wright, J.P./Blevins, K.R. (Hrsg.), Taking Stock. The Status of Criminological Theory (Advances in Criminological Theory, Vol. 15). New Brunswick: Transaction.

Lauritsen, Janet L., 2001: The Social Ecology of Violent Victimization: Individual and Contextual Effects in the NCVS. Journal of Quantitative Criminology 17/1: 3-32.

Lauritsen, Janet L./Laub, John H., 2007: Understanding the Link between Victimization and Offending. New Reflections on an Old Idea. S. 55-76 in: Hough, M./Maxfield, M. (Hrsg.), Surveying Crime in the 21st Century. Commemorating the 25th Anniversary of the British Crime Survey Monsy. New York: Criminal Justice Press.

Lauritsen, Janet L./White, Norman A., 2001: Putting Violence in its Place: The Influence of Race, Ethnicity, Gender, and Place on the Risk for Violence. Criminology and Public Policy 1/1: 37-60.

Lautmann, Rüdiger/Klimke, Daniela, 2004: Punitivität als Schlüsselbegriff für eine Kritische Kriminologie. S. 929 in: Lautmann, R./Klimke, D./Sack, F. (Hrsg.), Punitivität (Kriminologisches Journal, 8. Beiheft). Weinheim: Juventa.

Lautmann, Rüdiger/Klimke, Daniela/Sack, Fritz (Hrsg.), 2004: Punitivität (Kriminologisches Journal, 8. Beiheft). Weinheim: Juventa.

Lee, Royce/Coccaro, Emil F., 2007: Neurobiology of Impulsive Aggression. Focus on Serotonin and the Orbitofrontal Cortex. S. 170-186 in: Flannery, D.J./Vazsonyi, A.T./Waldman, I.D. (Hrsg.), The Cambridge Handbook of Violent Behavior and Aggression. Cambridge: Cambridge University Press.

Legewie, Joscha/Simons, Arne, 2009: Zur Rationalität von Selbstmordattentätern. Kritische Überlegungen zur Anwendung von Rational-Choice Theorien. S. 254-274 in: Shikano, S./Behnke, J./Bräuninger, T. (Hrsg.), Jahrbuch für Handlungs- und Entscheidungstheorie, Band 5: Theorien der Verfassungsreform. Wiesbaden: VS Verlag für Sozialwissenschaften.

Lemert, Edwin M., 1967: Human Deviance: Social Problems and Social Control. Englewood Cliffs, N.J.: Prentice Hall.

Lemert, Edwin M., 1974: Beyond Mead: The Societal Reaction to Deviance. Social Problems 21/4: 457-468.

Lemert, Edwin M., 1981: Diversion in Juvenile Justice: What Hath been Wrought. Journal of Research in Crime and Delinquency 18: 34-46.

Leps, Marie-Christine, 1992: Apprehending the Criminal. The Production of Deviance in the Nineteenth-Century Discourse. Durham, NC: Duke University Press.

Levitt, Steven D., 1997: Using Electoral Cycles in Police Hiring to Estimate the Effect of Police on Crime. The American Economic Review 87/3: 270-290.

Levitt, Steven D., 1998: Juvenile Crime and Punishment. Journal of Political Economy 106: 1156-1185.

Liberman, Akiva (Hrsg.), 2008: The Long View of Crime. A Synthesis of Longitudinal Research. New York: Springer.

Linebaugh, Peter, 2003: The London Hanged. Crime and Civil Society in the Eighteenth Century. London: Verso.

Loader, Ian/Walker, Neil, 2007: Civilizing Security. Cambridge: Cambridge University Press.

Loeber, Rolf/Farrington, David P./Waschbusch, Daniel A., 1998: Serious and Violent Juvenile Offenders. S. 13-29 in: Loeber, R./Farrington, D.P. (Hrsg.), Serious and Violent Juvenile Offenders. Risk Factors and Successful Interventions. Thousand Oaks.: Sage.

Loeber, Rolf/Pardini, Dustin, 2009: Neurobiology and the Development of Violence. Common Assumptions and Controversies. Monatsschrift für Kriminologie und Strafrechtsreform 92/2/3: 206-225.

Loeber, Rolf/Pardini, Dustin/Homish, D. L./Wei, Evelyn H./Crawford, Anne M./Farrington, David P./StouthamerLoeber, Magda/Creemers, Judith/Koehler, Steven A./Rosenfeld, Richard, 2005: The Prediction of Violence and Homicide in Young Men. Journal of Consulting and Clinical Psychology 73/6: 1074-1088.

Lösel, Friedrich, 1975: Handlungskontrolle und Jugenddelinquenz. Persönlichkeitspsychologische Erklärungsansätze delinquenten Verhaltens - theoretische Integration und empirische Prüfung. Stuttgart: Enke.

Lösel, Friedrich/Bliesener, Thomas, 2003: Aggression, Gewalt und Delinquenz unter Jugendlichen. Neuwied: Luchterhand.

Lösel, Friedrich/Bliesener, Thomas/Averbeck, Mechthild, 1998: Hat die Delinquenz von Schülern zugenommen? Ein Vergleich im Dunkelfeld nach 22 Jahren? DVJJ-Journal 9: 115-125. 
Lüdemann, Christian, 2000: Normen, Sanktionen und soziale Kontrolle in der Theorie rationalen Handelns von James S. Coleman. S. 87-110 in: Peters, H. (Hrsg.), Soziale Kontrolle. Zum Problem der Normkonformität in der Gesellschaft. Opladen: Westdeutscher Verlag.

Lüdemann, Christian, 2005: Benachteiligte Wohngebiete, lokales Sozialkapital und „Disorder“. Eine Mehrebenenanalyse zu den individuellen und sozialräumlichen Determinanten der Perzeption von Physical und Social Incivilities im städtischen Raum. Monatsschrift für Kriminologie und Strafrechtsreform 88/4: 240-256.

Lüdemann, Christian, 2006: Kriminalitätsfurcht im urbanen Raum. Eine Mehrebenenanalyse zu individuellen und sozialräumlichen Determinanten verschiedener Dimensionen von Kriminalitätsfurcht. Kölner Zeitschrift für Soziologie und Sozialpsychologie 58/2: 285-306.

Lüdemann, Christian/Ohlemacher, Thomas, 2002: Soziologie der Kriminalität. Theoretische und empirische Perspektiven. Weinheim: Juventa.

Ludwig-Mayerhofer, Wolfgang (Hrsg.), 2000: Soziale Ungleichheit, Kriminalität und Kriminalisierung. Opladen: Leske + Budrich.

Lynam, Donald R./Caspi, Avshalom/Moffitt, Terrie E./Wikström, Per-Olof H./Loeber, Rolf/Novak, Scott, 2000 : The Interaction Between Impulsivity and Neighborhood Context on Offending. The Effects of Impulsivity are Stronger in Poorer Neighborhoods. Journal of Abnormal Psychology 109/4: 563-574.

Lynch, James P., 2006: Problems and Promise of Victimization Surveys for Cross-National Research. S. 229-287 in: Tonry, M. (Hrsg.), Crime and Justice. A Review of Research, Vol. 34. Chicago: Chicago University Press.

Lynch, James P./Addington, Lynn A., 2007: Understanding Crime Statistics. Revisiting the Divergence of the NCVS and UCR. Cambridge: Cambridge University Press.

Mahoney, Joseph L./Stattin, Hakan/Lord, Heather, 2004: Unstructured Youth Recreation Centre Participation and Antisocial Behaviour Development. Selection Influences and the Moderating Role of Antisocial Peers. International Journal of Behavioral Development 28/6: 553-560.

Mansel, Jürgen/Hurrelmann, Klaus, 1998: Aggressives und delinquentes Verhalten Jugendlicher im Zeitvergleich, Befunde der Dunkelforschung aus den Jahren 1988, 1990 und 1996. Kölner Zeitschrift für Soziologie und Sozialpsychologie 50/1: 78-109.

Markowitsch, Hans J./Siefer, Werner, 2007: Tatort Gehirn. Auf der Suche nach dem Ursprung des Verbrechens. Frankfurt/M.: Campus.

Markowitz, Fred E./Bellair, Paul E./Liska, Allen E./Liu, Jianhong, 2001: Extending Social Disorganization Theory. Modeling the Relationships between Cohesion, Disorder, and Fear. Criminology 39/2: 293-319.

Maruna, Shadd, 2001: Making Good: How Ex-Convicts Reform and Rebuild Their Lives. Washington, DC: American Psychological Assocation Books.

Maruna, Shadd/Farrall, Stephen, 2004: Desistance from Crime: A Theoretical Reformulation. S. 171-194 in: Oberwittler, D./Karstedt, S. (Hrsg.), Soziologie der Kriminalität (Sonderheft 43 der Kölner Zeitschrift für Soziologie und Sozialpsychologie). Wiesbaden: VS - Verlag für Sozialwissenschaften.

Maruna, Shadd/King, Anna, 2004: Public Opinion and Community Sanctions. S. 83-112 in: Bottoms, A.E./Rex, S. (Hrsg.), Alternatives to Prisons. Options for an Insecure Society. Cullompton: Willan.

Maruna, Shadd/Matravers, Amanda/King, Anna, 2004: Disowning our Shadow. A Psychoanalytic Approach to Understanding Punitive Public Attitudes. Deviant Behavior 25: 277-299.

Mascini, Peter/Houtman, Dick, 2006: Rehabilitation and Repression. Reassessing their Ideological Embeddedness. British Journal of Criminology 46: 822-836.

Massey, Douglas S., 1990: American Apartheid. Segregation and the Making of the Underclass. American Journal of Sociology 96/2: 329-357.

Matsueda, Ross L., 2006: Criminological Implications of the Thought of George Herbert Mead. S. 77-108 in: Deflem, M. (Hrsg.), Sociological Theory and Criminological Research. Views from Europe and the United States. Amsterdem: Elsevier.

Matsueda, Ross L./Anderson, Kathleen, 1998: The Dynamics of Delinquent Peers and Delinquent Behavior. Criminology 36/2: 269-308.

Matsueda, Ross L./Kreager, Derek A./Huizinga, David, 2006: Deterring Delinquents. A Rational Choice Model of Theft and Violence. American Sociological Review 71: 85-122.

Matza, David, 1990: Delinquency and Drift. New Brunswick: Transaction. [zuerst 1964].

Matza, David/Sykes, Gesham M., 1961: Juvenile Delinquency and Subterranean Values. American Sociological Review 26/5: 712-719.

Mayhew, Henry, 1861: London Labour and the London Poor. The Condition and Earnings of those that will Work, those that cannot Work, and those that will not Work. London: Griffin.

McCarthy, Bill, 2002: New Economics of Sociological Criminology. Annual Review of Sociology 28: 417-442.

McClure, Samuel M./Laibson, David I./Loewenstein, George/Cohen, Jonathan D., 2004: Separate Neural Systems Value Immediate and Delayed Monetary Rewards. Science 306: 503-507. 
McGowan, Patrick O./Sasaki, Aya/D"eAlessio, Ana C./Dymov, Sergiy/Labonté, Benoit/Szyf, Moshe/Turecki, Gustavo/Meaney, Michael J., 2009: Epigenetic Regulation of the Glucocorticoid Receptor in Human Brain Associates with Childhood Abuse. Nature Neuroscience 12/3: 342-348.

Mead, George H., 1918: The Psychology of Punitive Justice. The American Journal of Sociology XXIII/5: 577 602 .

Meany, Michale J., 2010: Epigenetics and the Biological Definition of Gene x Environment Interactions. Child Development 81/1: 41-79.

Mehlum, Halvor/Miguel, Edward/Ragnar, Torvik, 2006: Poverty and Crime in 19th Century Germany. Journal of Urban Economics 59/3: 370-388

Mehlum, Halvor/Moene, Karl/Ragnar, Torvik, 2005: Crime Induced Poverty Traps. Journal of Development Economics 77: 325-340.

Melchers, Alexander, 1992: Kriminalstatistik im 19. Jahrhundert. Ein Beitrag zur Geschichte der Kriminalsoziologie und ihrer Methodik (Dissertation Universität Frankfurt). Frankfurt/M.: Universität Frankfurt.

Meldrum, Ryan C./Young, Jacob T. N./Weerman, Frank M., 2009: Reconsidering the Effect of Self-Control and Delinquent Peers Implications of Measurement for Theoretical Significance. Journal of Research in Crime and Delinquency 46/3: 353-376.

Melossi, Dario, 2001: Changing Representations of the Criminal. S. 9-44 in: Albrecht, H.-J./Koukoutsaki, A./Serassis, T. (Hrsg.), Images of Crime. Freiburg: edition iuscrim.

Menzel, Birgit/Peters, Helge, 2003: Sexuelle Gewalt. Eine definitionstheoretische Untersuchung. Konstanz: Universitätsverlag.

Merton, Robert K., 1938: Social Structure and Anomie. American Sociological Review 3: 672-682.

Messner, Steven F., 2002: Gewaltkriminalität im Ländervergleich. S. 875-895 in: Heitmeyer, W./Hagan, J. (Hrsg.), Internationales Handbuch der Gewaltforschung. Wiesbaden: Westdeutscher Verlag.

Messner, Steven F., 2004: An Institutional-Anomie Theory of Crime: Continuities and Elaborations in the Study of Social Structure and Anomie. S. 93-109 in: Oberwittler, D./Karstedt, S. (Hrsg.), Soziologie der Kriminalität (Sonderheft 43 der Kölner Zeitschrift für Soziologie und Sozialpsychologie). Wiesbaden: VS - Verlag für Sozialwissenschaften.

Messner, Steven F./Rosenfeld, Richard, 1994: Crime and the American Dream [2. Auflage]. Belmont, Cal.: Wadsworth.

Messner, Steven F./Rosenfeld, Richard, 2006: The Present and Future of Institutional-Anomie Theory. S. 127-148 in: Cullen, F.T./Wright, J.P./Blevins, K.R. (Hrsg.), Taking Stock. The Status of Criminological Theory (Advances in Criminological Theory, Vol. 15). New Brunswick: Transaction.

Messner, Steven F./Thome, Helmut/Rosenfeld, Richard, 2008: Institutions, Anomie, and Violent Crime. Clarify- ing and Elaborating Institutional-Anomie Theory. International Journal of Conflict and Violence 2/2: 163-181.

Meulemann, Heiner, 2003: Der Sozialstaat in der amtlichen Statistik. Angebote und Nachfragen. Bonn: Informationszentrum Sozialwissenschaften.

Miller, Walter B., 1958: Lower Class Culture as a Generating Milieu of Gang Delinquency. Journal of Social Issues 14: 5-19.

Mischel, Walter, 1974: Processes in the Delay of Gratification. S. 249-293 in: Berkowitz, L. (Hrsg.), Advances in Experimental Social Psychology, Vol. 8. New York: Academic Press.

Mischel, Walter/Shoda, Yuichi/Rodriguez, Monica, 1989: Delay of Gratification in Children. Science 244: 933938

Mischkowitz, Robert, 1993: Kriminelle Karrieren und ihr Abbruch. Empirische Ergebnisse einer kriminologischen Langzeituntersuchung als Beitrag zur „Age-Crime Debate“. Bonn: Forum Verlag Godesberg.

Mocan, Naci H./Rees, Daniel I., 2005: Economic Conditions, Deterrence and Juvenile Crime: Evidence vom Micro Data. American Law and Economics Review 7/2: 319-349.

Moffitt, Terrie E., 1993: Adolescence-Limited and Life-Course-Persistent Antisocial Behavior. A Developmental Taxonomy. Psychological Review 100: 674-701.

Moffitt, Terrie E., 2005: The New Look of Behavioral Genetics in Developmental Psychopathology. Gene Environment Interplay in Antisocial Behaviors. Psychological Bulletin 131/4: 533-554

Moffitt, Terrie E., 2006: A Review of Research on the Taxonomy of Life-Course-Persistent versus AdolescentLimited Antisocial Behavior. S. 277-312 in: Cullen, F.T./Wright, J.P./Blevins, K.R. (Hrsg.), Taking Stock. The Status of Criminological Theory (Advances in Criminological Theory, Vol. 15). New Brunswick: Transaction.

Moffitt, Terrie E./Caspi, Avshalom, 2001: Childhood Predictors Differentiate Life-Course Persistent and Adolescence-Limited Antisocial Pathways among Males and Females. Development and Psychopathology 13: 355375. 
Moffitt, Terrie E./Caspi, Avshalom, 2003: Preventing the Inter-Generational Continuity of Anti-Social Behavior. Implications of Partner Violence. S. 109-129 in: Farrington, D.P./Coid, J. (Hrsg.), Early Prevention of Adult Antisocial Behaviour. Cambridge: Cambridge University Press.

Moffitt, Terrie E./Caspi, Avshalom/Harrington, Hona L./Milne, Barry J., 2002: Males on the Life-Course-Persistent and Adolescence-Limited Antisocial Pathways. Follow-Up at Age 26 Years. Development and Psychopathology 14: 179-207.

Morenoff, Jeffrey D./Sampson, Robert J., 1997: Violent Crime and the Spatial Dynamics of Neighborhood Transition: Chicago, 1970-1990. Social Forces 76/1: 31-64.

Müller, Ursula/Schröttle, Monika, 2004: Lebenssituation, Sicherheit und Gesundheit von Frauen in Deutschland. Eine repräsentative Untersuchung zu Gewalt gegen Frauen in Deutschland. Berlin: Bundesministerium für Familie, Senioren, Frauen und Jugend.

Murck, Manfred, 1978: Die Angst vor Verbrechen und Einstellungen zu Problemen der öffentlichen Sicherheit. Kriminologisches Journal 10/3: 202-214.

Nagin, Daniel S., 1998: Criminal Deterrence Research at the Outset of Twenty-First Century. S. 1-42 in: Tonry, M. (Hrsg.), Crime and Justice. A Review of Research, Vol. 23. Chicago: University of Chicago Press.

Nagin, Daniel S., 1999: Analyzing Developmental Trajectories. A Semiparametric, Group-Based Approach. Psychological Methods 4/2: 139-157.

Nagin, Daniel S., 2005: Group-Based Modeling of Development. Cambridge, Mass.: Havard University Press.

Nagin, Daniel S./Tremblay, Richard E., 2005: Developmental Trajectory Groups: Fact or a Useful Statistical Fiction? Criminology 43/4: 873-904.

Naplava, Thomas, 2005: Jugenddelinquenz im interethnischen Vergleich. Erklärungsmöglichkeiten delinquenten Verhaltens einheimischer und immigrierter Jugendlicher (Dissertation). Bielefeld: Fakultät für Soziologie. Universität Bielefeld. Internetquelle: [http://nbn-resolving.de/urn/resolver.pl?urn=urn:nbn:de:hbz:361-10128]

Naplava, Thomas/Oberwittler, Dietrich, 2002: Methodeneffekte bei der Messung selbstberichteter Delinquenz von männlichen Jugendlichen. Ein Vergleich zwischen schriftlicher Befragung in der Schule und mündlicher Befragung im Haushalt. Monatsschrift für Kriminologie und Strafrechtsreform 85/6: 401-423.

Newburn, Tim/Jones, Trevor, 2005: Symbolic Politics and Penal Populism. The Long Shadow of Willie Horton. Crime, Media, Culture 1/1: 72-87.

Nicholas, Sian/Kershaw, Chris/Walker, Alison, 2007: Crime in England and Wales 2006/07 (Home Office Statistical Bulletin 2006/7). London: Home Office. Internetquelle: [http://rds.homeoffice.gov.uk/rds/pdfs07/hosb11 07.pdf].

Obergfell-Fuchs, Joachim/Kury, Helmut, 1996: Sicherheitsgefühl und Persönlichkeit. Monatsschrift für Kriminologie und Strafrechtsreform 79/2: 97-113.

Oberwittler, Dietrich, 2004a: Re-Balancing Routine Activity and Social Disorganization Theories in the Explanation of Urban Violence. A New Approach to the Analysis of Spatial Crime Patterns Based on Population at Risk (Working Paper 10 für das Projekt "Social Problems and Juvenile Delinquency in Ecological Perspective"). Freiburg i. Br. (unveröffentlichtes Manuskript).

Oberwittler, Dietrich, 2004b: Stadtstruktur, Freundeskreise und Delinquenz. Eine Mehrebenenanalyse zu sozialökologischen Kontexteffekten auf schwere Jugenddelinquenz. S. 135-170 in: Oberwittler, D./Karstedt, S. (Hrsg.), Soziologie der Kriminalität (Sonderheft 43 der Kölner Zeitschrift für Soziologie und Sozialpsychologie). Wiesbaden: VS - Verlag für Sozialwissenschaften.

Oberwittler, Dietrich, 2007: The Effects of Neighbourhood Poverty on Adolescent Problem Behaviours: A Multilevel Analysis Differentiated by Gender and Ethnicity. Housing Studies 22/5: 781-803.

Oberwittler, Dietrich, 2008: Armut macht Angst. Ansätze einer sozialökologischen Interpretation der Kriminalitätsfurcht. S. 215-230 in: Groenemeyer, A./Wieseler, S. (Hrsg.), Soziologie sozialer Probleme und sozialer Kontrolle. Realitäten, Repräsentationen und Politik. Festschrift für Günter Albrecht. Wiesbaden: VS - Verlag für Sozialwissenschaften.

Oberwittler, Dietrich, 2010: Jugendkriminalität in sozialen Kontexten. Zur Rolle von Wohngebieten und Schulen bei der Verstärkung von abweichendem Verhalten Jugendlicher. S. 213-227 in: Dollinger, B./Schmidt-Semisch, H. (Hrsg.), Handbuch Jugendkriminalität. Kriminologie und Sozialpädagogik im Dialog. Wiesbaden: VS - Verlag für Sozialwissenschaften.

Oberwittler, Dietrich/Blank, Thomas/Köllisch, Tilman/Naplava, Thomas, 2001: Soziale Lebenslagen und Delinquenz von Jugendlichen. Ergebnisse der MPI-Schulbefragung 1999 in Freiburg und Köln (Arbeitsberichte aus dem Max-Planck-Institut für ausländisches und interationales Strafrecht). Freiburg i. Br.: edition iuscrim. Internetquelle: [http://www.iuscrim.mpg.de/shared/data/pdf/a1.pdf].

Oberwittler, Dietrich/Hofer, Sven, 2005: Crime and Justice in Germany. An Analysis of Recent Trends and Research. European Journal of Criminology 2/4: 465-508. 
Oberwittler, Dietrich/Köllisch, Tilman, 2004: Nicht die Jugendgewalt, sondern deren polizeiliche Registrierung hat zugenommen. Ergebnisse einer Vergleichsstudie nach 25 Jahren. Neue Kriminalpolitik 16/4: 81-120.

Oberwittler, Dietrich/Lukas, Tim, 2010: Schichtbezogene und ethnisierende Diskriminierung im Prozess der strafrechtlichen Sozialkontrolle. S. 221-254 in: Scherr, A./Hormel, U. (Hrsg.), Diskriminierung: Grundlagen und Forschungsergebnisse. Wiesbaden: VS - Verlag für Sozialwissenschaften.

Oberwittler, Dietrich/Naplava, Thomas, 2002: Auswirkungen des Erhebungsverfahrens bei Jugendbefragungen zu heiklen Themen. Schulbasierte schriftliche Befragung und haushaltsbasierte mündliche Befragung im Vergleich. ZUMA-Nachrichten 51: 49-77.

Oberwittler, Dietrich/Rabold, Susann/Baier, Dirk, 2011: Städtische Armutsquartiere - Kriminelle Lebenswelten? Studien zu sozialräumlichen Kontexteffekten auf Jugendkriminalität und Kriminalitätswahrnehmungen. Wiesbaden: VS - Verlag für Sozialwissenschaften.

Oberwittler, Dietrich/Wikström, Per-Olof H., 2006: Behavioural Contexts and Victimization (Presentation at the ASC Annual Meeting 2006 in Los Angeles). (unveröffentlichtes Manuskript):

O"Brian, Robert, 2003: UCR Violent Crime Rates, 1958-2000: Recorded and Offender-Generated Trends. Social Science Research 32: 499-518.

Osgood, D. W./Anderson, Amy L., 2004: Unstructured Socializing and Rates of Delinquency. Criminology 42/3: 519-549.

Oswald, Margit E./Hupfeld, Jörg/Klug, Stefan/Gabriel, Ute, 2002: Lay-Perspectices on Criminal Deviance, Goals of Punishment, and Punitivity. Social Justice Research 15/2: 85-98

Pearson, Geoffrey, 1984: Hooligan. A History of Respectable Fears. London: MacMillan.

Perkins, D.D./Taylor, Ralph B., 1996: Ecological Assessments of Community Disorder. Their Relationship to Fear of Crime and Theoretical Implications. American Journal of Community Psychology 24/1: 63-107.

Peters, Helge, 1996: Als Partisanenwissenschaft ausgedient, als Theorie aber nicht sterblich: der Labeling Approach. Kriminologisches Journal 28/2: 107-115.

Peters, Helge, 2009: Devianz und soziale Kontrolle. Eine Einführung in die Soziologie abweichenden Verhaltens. (3. Auflage). Weinheim: Juventa.

Peterson, Ruth D./Krivo, Lauren J./Hagan, John, 2006: The Many Colors of Crime. Inequalities of Race, Ethnicity, and Crime in America. New York: New York University Press.

Pfeiffer, Christian/Delzer, Ingo/Enzmann, Dirk/Wetzels, Peter, 1998: Ausgrenzung, Gewalt und Kriminalität im Leben junger Menschen.Kinder und Jugendliche als Opfer und Täter. (DVJJ, Sonderdruck zum 24. Deutschen Jugendgerichtstag vom 18.-22. September 1998 in Hamburg). Hamburg.

Pfeiffer, Christian/Windzio, Michael/Kleimann, Matthias, 2004: Die Medien, das Böse und wir. Zu den Auswirkungen der Mediennutzung auf Kriminalitätswahrnehmung, Strafbedürfnisse und Kriminalpolitik. Monatsschrift für Kriminologie und Strafrechtsreform 87/6: 415-435.

Pfeiffer, Christian/Windzio, Michael/Kleimann, Matthias, 2005: Media Use and its Impacts on Crime Perception, Sentencing Attitudes and Crime Policy. European Journal of Criminology 2/3: 259-285.

Pfeiffer, Dietmar/Scheerer, Sebastian, 1979: Kriminalsoziologie. Einführung in Theorien und Themen. Stuttgart: Kohlhammer.

Piquero, Alex R./Farrington, David P./Blumstein, Alfred, 2007: Key Issues in Criminal Career Research. New Analyses of the Cambridge Study in Delinquent Development. Cambridge: Cambridge University Press.

Pitts, John, 2008: Reluctant Gangsters. The Changing Face of Youth Crime. Cullompton: Willan.

Pratt, John, 2007: Penal Populism. London: Routledge.

Pratt, John/Brown, David/Brown, Mark/Hallsworth, Simon/Morrison, Wayne, 2005: The Pew Punitiveness. Trends, Theories, Perspectives. Cullompton: Willan.

Pratt, Travis C./Cullen, Francis T., 2000: The Empirical Status of Gottfredson and Hirschies General Theory of Crime: A Meta-Analysis. Criminology 38/3: 931-964.

Pratt, Travis C./Cullen, Francis T., 2005: Assessing Macro-Level Predictors and Theories of Crime. A Meta-Analysis. S. 373-450 in: Tonry, M. (Hrsg.), Crime and Justice. An Annual Review of Research, Vol. 32.32. Jg., Heft Chicago: Chicago University Press.

Pratt, Travis C./Cullen, Francis T./Blevins, Kristie R./Daigle, Leah E./Madensen, Tamara D., 2006: The Empirical Status of Deterrence Theory. S. 367-396 in: Cullen, F.T./Wright, J.P./Blevins, K.R. (Hrsg.), Taking Stock. The Status of Criminological Theory, Vol. 15. Roxbury: Transaction.

Pratt, Travis C./Cullen, Francis T./Blevins, Kristie R./Daigle, Leah/Unnever, James D., 2002: The Relationship of Attention Deficit Hyperactivity Disorder to Crime and Delinquency. A Meta-Analysis. International Journal of Police Science and Management 4/4: 344-360.

Pridemore, William A., 2002: What We Know About Social Structure and Homicide. A Review of the Theoretical and Empirical Literature. Violence and Victims 17/2: 127-156. 
Pridemore, William A./Chamlin, Mitchell B./Cochran, John K., 2007: An Interrupted Time-Series Analysis of Durkheim 'es Social Deregulation Thesis: The Case of the Russian Federation. Justice Quarterly 24/2: 271-290. Pridemore, William A./Kim, Sang-Weon, 2007: Socioeconomic Change and Homicide in a Transitional Society. Sociological Quarterly 48/2: 229-251.

Prinz, Wolfgang, 2008: Der Wille als Artefakt. S. 642-655 in: Rehberg, K.-S. (Hrsg.), Die Natur der Gesellschaft. Verhandlungen des 33. Kongresses der Deutschen Gesellschaft für Soziologie in Kassel 2006. Frankfurt/M.: Campus.

Quensel, Stephan, 2009: Wer raucht, der stiehlt ... Zur Interpretation quantitativer Daten in der Jugendsoziologie. Eine jugendkriminologische Studie. Wiesbaden: VS - Verlag für Sozialwissenschaften.

Quételet, Adolphe, 1921: Soziale Physik oder Abhandlung über die Entwicklung der Fähigkeiten des Menschen. Jena: Gustav Fischer [fr. org 1869: Physique sociale, ou, Essai sur le développement des facultés de l'homme. Bruxelles: C. Muquard].

Rabold, Susann/Baier, Dirk, 2007: Delinquentes Verhalten von Jugendlichen. Zur differentiellen Bedeutsamkeit verschiedener Bedingungsfaktoren. S. 9-42 in: GESIS (Hrsg.), soFid Sozialwissenschaftlicher Fachinformationsdienst Kriminalsoziologie + Rechtssoziologie 2007/2. Bonn: GESIS.

Raithel, Jürgen, (Hrsg.) 2001: Risikoverhaltensweisen Jugendlicher. Opladen: Leske + Budrich.

Raithel, Jürgen/Mansel, Jürgen, 2003: Kriminalität und Gewalt im Jugendalter. Hell- und Dunkelfeldbefunde im Vergleich. Weinheim: Juventa.

Ranulf, Svend, 1964: Moral Indignation and Middle Class Psychology. A Sociological Study [org. 1938]. New York: Schocken Books.

Raphael, Steven/Winter-Ebmer, Rudolf, 2001: Identifying the Effect of Unemployment on Crime. Journal of Law and Economics 44/1: 259-284.

Raudenbush, Stephen W./Sampson, Robert J., 1999: Ecometrics: Toward a Science of Assessing Ecological Settings, with Appliance to the Systematic Social Observation of Neighborhoods. Sociological Methodology 29: $1-41$.

Reinecke, Jost, 2008: Klassifikation von Delinquenzverläufen: Eine Anwendung mit Mischverteilungsmodellen. S. 189-218 in: Reinecke, J./Tarnai, C. (Hrsg.), Klassifikationsanalysen in Theorie und Praxis. Münster: Waxmann.

Reiner, Robert, 2007: Media-Made Criminality. The Representation of Crime in the Mass Media. S. $302-340$ in: Maguire, M./Morgan, R./Reiner, R. (Hrsg.), The Oxford Handbook of Criminology [4. Auflage]. Oxford: Oxford University Press.

Reuband, Karl H., 1992a: Kriminalitätsfurcht in Ost- und Westdeutschland. Zur Bedeutung psychosozialer Einflußfaktoren. Soziale Probleme 3/2: 211-219.

Reuband, Karl H., 1992b: Objektive und subjektive Bedrohung durch Kriminalität. Ein Vergleich der Kriminalitätsfurcht in der BRD und den USA 1965-1990. Kölner Zeitschrift für Soziologie und Sozialpsychologie 44/2: 341-353.

Reuband, Karl H., 1996: Gesellschaftlicher Wandel, Kriminalität und Kriminalitätsfurcht. Neue Praxis 26/6: 494504.

Reuband, Karl-Heinz, 1998: Kriminalität in den Medien. Erscheinungsformen, Nutzungsstruktur und Auswirkungen auf die Kriminalitätsfurcht. Soziale Probleme 9/2: 122-153.

Reuband, Karl-Heinz, 2002: Law and Order als neues Thema bundesdeutscher Politik? Wie es zum Wahlerfolg der Schill Partei in Hamburg kam und welche Auswirkungen dies hat. Neue Kriminalpolitik 1: 8-13.

Reuband, Karl-Heinz, 2004: Steigende Straflust der Bundesbürger? Ein Vergleich bundesweiter Umfragen zwischen 1970 und 2003. S. 89-103 in: Lautmann, R./Klimke, D./Sack, F. (Hrsg.), Punitivität (Kriminologisches Journal, 8. Beiheft). Weinheim: Juventa.

Reuband, Karl-Heinz, 2007: Steigende Kriminalitätsbedrohung, Medienberichterstattung und Kriminalitätsfurcht der Bürger. S. 71-86 in: Hess, H./Ostermeier, L./Paul, B. (Hrsg.), Kontrollkulturen. Texte zur Kriminalpolitik in Anschluss an David Garland (Kriminologisches Journal, 9. Beiheft). Weinheim: Juventa.

Reuter, Peter, 1983: Disorganized Crime. The Economics of the Visible Hand. Cambridge, Mass.: MIT Press.

Ribeaud, Denis/Eisner, Manuel, 2006: The Drug Crime Link from a Self-Control Perspective. An Empirical Test in a Swiss Youth Sample. European Journal of Criminology 3/1: 33-67.

Ribeaud, Denis/Eisner, Manuel, 2009: Entwicklung von Gewalterfahrungen Jugendlicher im Kanton Zürich (1999-2007). Oberentfelden: Sauerländer.

Richter, Harald, 1997: Opfer krimineller Gewalttaten. Individuelle Folgen und ihre Verarbeitung. Ergebnisse einer Untersuchung. Mainz: Weißer Ring.

Rippl, Susanne/Baier, Dirk, 2005: Das Deprivationskonzept in der Rechtsextremismusforschung. Eine vergleichende Analyse. Kölner Zeitschrift für Soziologie und Sozialpsychologie 57/4: 644-666. 
Robert, Philippe (Hrsg.), 2009:. Comparing Crime Data in Europe. Official Crime Statistics and Survey-Based Data. Brüssel: VUB Press.

Roberts, Julian V./Stalans, Loretta J./Indermaur, David/Hough, Mike, 2003: Penal Populism and Public Opinion. Lessons from Five Countries. Oxford: Oxford University Press.

Robinson, Jennifer B./Lawton, Brian A./Taylor, Ralph B./Perkins, Douglas D., 2003: Multilevel Longitudinal Impacts of Incivilities. Fear of Crime, Expected Safety, and Block Satisfaction. Journal of Quantitative Criminology 19/3: 237-274.

Roe, Stephen/Ashe, Jane, 2008: Young People and Crime. Findings from the 2006 Offending, Crime and Justice Survey (Home Office Statistical Bulletin 09/08). London: Home Office. Internetquelle: [http://rds.homeoffice.gov.uk/rds/pdfs08/hosb0908.pdf].

Roncek, Dennis W./Maier, Pamela A., 1991: Bars, Blocks and Crimes Revisited. Linking the Theory of Routine Activities to the Empiricism of Hot Spots. Criminology 29/4: 725-753.

Roshier, Bob, 1977: The Function of Crime Myth. The Sociological Review 25: 309-323.

Ross, Catherine E./Mirowsky, John/Pribesh, Shana, 2001: Powerlessness and the Amplification of Threat. Neighborhood Disadvantage, Disorder, and Mistrust. American Sociological Review 66: 568-591.

Roth, Gerhard, 2006: Willensfreiheit und Schuldfähigkeit aus Sicht der Hirnforschung. S. 9-28 in: Roth, G./Grün, K.-J. (Hrsg.), Das Gehirn und seine Freiheit. Beiträge zur neurowissenschaftlichen Grundlegung der Philosophie. Göttingen: Vandenhoeck \& Ruprecht.

Roth, Gerhard/Lück, Monika/Strüber, Daniel, 2006: „Freier Wille“ und Schuld von Gewaltstraftätern aus Sicht der Hirnforschung und Neuropsychologie. Neue Kriminalpolitik 2: 55-59.

Rountree, Pamela W./Land, Kenneth C./Miethe, Terance D., 1994: Macro-Micro Integration in the Study of Victimization. A Hierarchical Logistic Model Analysis Across Seattle Neighborhoods. Criminology 32/3: 387-414. Rutter, Michael/Silberg, Judy, 2002: Gene-Environment Interplay in Relation to Emotional and Behavioral Disturbance. Annual Review of Psychology 53: 463-490.

Sack, Fritz, 1968: Neue Perspektiven in der Kriminologie. S. 431-475 in: Sack, F./König, R. (Hrsg.), Kriminalsoziologie. Frankfurt/M.: Akademische Verlagsanstalt.

Sack, Fritz, 1969: Probleme der Kriminalsoziologie. S. 961-1049 in: König, R. (Hrsg.), Handbuch der empirischen Sozialforschung, Band 2. Stuttgart: Ferdinand Enke.

Sack, Fritz, 2007: Die deutsche Kriminologie - von „draußen“ betrachtet. S. 205-230 in: Hess, H./Ostermeier, L./ Paul, B. (Hrsg.), Kontrollkulturen. Texte zur Kriminalpolitik im Anschluss an David Garland (Kriminologisches Journal, 9. Beiheft). Weinheim: Juventa.

Sack, Fritz, 2008: Die deutsche Kriminologie im Lichte des Werkes von D. Garland. S. 301-322 in: Klimke, D. (Hrsg.), Exklusion in der Marktgesellschaft. Wiesbaden: VS - Verlag für Sozialwissenschaften.

Sameroff, Arnold, 2010: A Unified Theory of Development. A Dialectic Integration of Nature and Nurture. Child Development 81/1: 6-22.

Sampson, Robert J., 1999: Techniques of Research Neutralization (Review Symposium on R. Akers, Social Learing and Social Structure: A General Theory of Crime and Deviance, Boston 1998). Theoretical Criminology 3/4: 438-451.

Sampson, Robert J., 2001: Artikel: "Delinquency, Sociology of". S. 3380-3384 in: Smelser, N.J./Baltes, P.L. (Hrsg.), International Encyclopedia of the Social and Behavioral Sciences. Amsterdam: Elsevier.

Sampson, Robert J./Groves, Walter B., 1989: Community Structure and Crime. Testing Social-Disorganization Theory. American Journal of Sociology 94/4: 774-831.

Sampson, Robert J./Laub, John H., 1993: Crime in the Making. Pathways and Turning Points Through Life. Cambridge, Mass: Harvard University Press.

Sampson, Robert J./Laub, John H., 2003: Life-Course Desisters? Trajectories of Crime Among Delinquent Boys Followed To Age 70. Criminology 41/3: 555-592.

Sampson, Robert J./Laub, John H., 2005: A Life-Course View of the Development of Crime. Annals of the American Academy of Political and Social Sciences 602: 12-45.

Sampson, Robert J./Laub, John H., 2009: A Life-Course Theory and Long-Term Project on Trajectories of Crime. Monatsschrift für Kriminologie und Strafrechtsreform 92/2/3: 226-239.

Sampson, Robert J./Morenoff, Jeffrey D./Gannon-Rowley, Thomas, 2002: Assessing "Neighborhood Effects". Social Processes and New Directions in Research. Annual Review of Sociology 28: 443-478.

Sampson, Robert J./Raudenbush, Stephen W., 1999: Systematic Social Observation of Public Spaces. A New Look at Disorder in Urban Neighborhoods. American Journal of Sociology 105/3: 603-651.

Sampson, Robert J./Raudenbush, Stephen W./Earls, Felton, 1997: Neighborhoods and Violent Crime. A Multilevel Study of Collective Efficacy. Science 277: 918-924.

Sampson, Robert J./Wilson, William J., 1995: Towards a Theory of Race, Crime, and Urban Inequality. S. 37-54 in: Hagan, J./Peterson, R.D. (Hrsg.), Crime and Inequality. Stanford, Cal.: Stanford University Press. 
Savelsberg, Joachim J., 2004: Religion, Historical Contingencies, and Institutional Conditions of Criminal Punishment. The German Case and Beyond. Law \& Social Inquiry 29/2: 373-401.

Savelsberg, Joachim J./Sampson, Robert J., 2002: Mutual Engagement? Criminology and Sociology (Introduction). Crime, Law \& Social Change 37: 99-105.

Scheerer, Sebastian, 1978: Der politisch-publizistische Verstärkerkreislauf. Zur Beeinflussung der Massenmedien im Prozeß strafrechtlicher Normgenese. Kriminologisches Journal 10: 223-227.

Schetsche, Michael, 2008: Empirsche Analyse sozialer Probleme. Das wissenssoziologische Programm. Wiesbaden: VS - Verlag für Sozialwissenschaften.

Schneider, Hans J., 2007: Viktimologie. S. 395-434 in: Schneider, H.J. (Hrsg.), Internationales Handbuch der Kriminologie, Band 1: Grundlagen der Kriminologie.1. Jg., Heft Berlin: de Gruyter.

Schnell, Rainer, 1997: Nonresponse in Bevölkerungsumfragen. Ausmaß, Entwicklung und Ursachen. Opladen: Leske + Budrich.

Schoepfer, Andrea/Piquero, Alex R., 2006: Self-Control, Moral Beliefs, and Criminal Activity. Deviant Behavior 27: 51-71.

Schumann, Karl F. (Hrsg.), 2003a: Berufsbildung, Arbeit und Delinquenz. Bremer Längsschnittstudie zum Übergang von der Schule in den Beruf bei ehemaligen Hauptschülern, Band 1. Weinheim: Juventa.

Schumann, Karl F. (Hrsg.), 2003b: Delinquenz im Lebenslauf. Bremer Längsschnittstudie zum Übergang von der Schule in den Beruf bei ehemaligen Hauptschülern, Band 2. Weinheim: Juventa.

Schumann, Karl F., 2010: Jugenddelinquenz im Lebenslauf. S. 243-258 in: Dollinger, B./Schmidt-Semisch, H. (Hrsg.), Handbuch Jugendkriminalität. Kriminologie und Sozialpädagogik im Dialog. Wiesbaden: VS - Verlag für Sozialwissenschaften.

Schumann, Karl F./Huizinga, David/Ehret, Beate/Elliott, Amanda, 2009: Cross-National Findings about the Effect of Job Training, Gangs, and Juvenile Justice Reactions on Delinquent Behavior and Desistance. Monatsschrift für Kriminologie und Strafrechtsreform 92/2-3: 309-326.

Schwind, Hans-Dieter/Fetchenhauer, Detlef/Ahlbom, Wilfried/Weiß, Rüdiger, 2001: Kriminalitätsphänomene im Langzeitvergleich am Beispiel einer deutschen Großstadt. Bochum 1975 - 1986 - 1998. Neuwied: Luchterhand.

Seipel, Christian/Eifler, Stefanie, 2004: Gelegenheiten, Rational Choice und Selbstkontrolle. Zur Erklärung abweichenden Handelns in High-Cost- und Low-Cost-Situationen. S. 288-315 in: Oberwittler, D./Karstedt, S. (Hrsg.), Soziologie der Kriminalität (Sonderheft 43 der Kölner Zeitschrift für Soziologie und Sozialpsychologie). Wiesbaden: VS - Verlag für Sozialwissenschaften.

Seo, Dongju/Patrick, Christopher J./Kennealy, Patrick J., 2008: Role of Serotonin and Dopamine System Interactions in the Neurobiology of Impulsive Aggression and its Comorbidity with other Clinical Disorders. Aggression and Violent Behavior 13: 383-395.

Sessar, Klaus, 1992: Wiedergutmachung oder Strafen. Einstellungen in der Bevölkerung und der Justiz. Pfaffenweiler: Centaurus.

Sessar, Klaus, 1997: Zu einer Kriminologie ohne Täter. Oder auch: Die kriminogene Tat. Monatsschrift für Kriminologie und Strafrechtsreform 80/1: 1-24.

Sessar, Klaus/Stangl, Wolfgang/van Swaaningen, René, (Hrsg.) 2007: Großstadtängst - Anxious Cities. Untersuchungen zu Unsicherheitsgefühlen und Sicherheitspolitiken in europäischen Kommunen. Münster: Lit.

Shaw, Clifford R./McKay, Henry D., 1969: Juvenile Delinquency and Urban Areas [2., revidierte Auflage, zuerst 1942]. Chicago: University of Chicago Press.

Shea, Evelyn, 2009: Elections and the Fear of Crime. The Case of France and Italy. European Journal on Criminal Policy and Research 15/1: 83-102.

Sherman, Lawrence W., 1997: Policing for Crime Prevention. S. 8/1-8/41 in: Sherman, L.W./Gottfredson, D./ Mackenzie, D./Eck, J./Reuter, P./Bushwa, S. (Hrsg.), Preventing Crime: What Works, What Doesn"t, What"s Promising. A Report to the United States Congress. College Park, MA. Internetquelle: [http://www.ncirs. gov/works/].

Sherman, Lawrence W./Gartin, Patrick R./Buerger, Micheal E., 1989: Hot Spots of Predatory Crime. Routine Activities and the Criminolgy of Place. Criminology 27/1: 27-55.

Sherman, Lawrence W./Weisburd, David, 1995: General Deterrent Effects of Police Patrol in Crime "Hot Spots". A Randomized, Controlled Trial. Justice Quarterly 12/4: 625-648.

Shonkoff, Jack P./Phillips, Deborah A., 2000: From Neurons to Neighborhoods. The Science of Early Childhood Development. Washington D.C.: National Acadamy Press.

Short, James F., 1997: Poverty, Ethnicity, and Violent Crime. Boulder, Col.: Westview.

Simmons, Jon/Legg, Clarissa/Hosking, Rachel, 2003: National Crime Recording Standard (NCRS). An Analysis of the Impact on Recorded Crime (On-Line Report 32/03). London: Home Office. Internetquelle: [http://rds. homeoffice.gov.uk/rds/pdfs2/rdsolr3203intro.pdf]. 
Simon, Jonathan, 2007: Governing Through Crime. How the War on Crime Formed American Democracy and Created a Culture of Fear. Oxford: Oxford University Press.

Sivarajasingam, Vaseekaran/Wells, John P./Moore, Simon/Shepherd, Jonathan P., 2009: Violence in England and Wales 2009. An Accident and Emergency Perspective (Violence and Society Research Group, Cardiff University). Internetquelle: [http://www.vrg.cf.ac.uk/nvit/NVIT_2009.pdf].

Skogan, Wesley G., 1990: Disorder and Decline. Crime and the Spiral of Decay in American Neighborhoouds. New York: Free Press.

Spitzer, Manfred/Fischbacher, Urs/Hermberger, Bärbel/Georg, Grön/Fehr, Ernst, 2007: The Neural Signature of Social Norm Compliance. Neuron 56: 185-196.

Spitzer, Steven, 1975: Punishment and Social Organization: A Study of Durkheim ees Theory of Penal Evolution. Law \& Society Review 9: 613-637.

Stadt Frankfurt/M., 2008: Die größten Stadtprobleme aus Sicht der Frankfurterinnen und Frankfurter. ERgebnisse der Frankfurter Bürgerbefragung vom Dezember 2007. statistik aktuell 08/2008. Statd Frankfurt/M. Internetquelle: [http://www.frankfurt.de/sixcms/media.php/678/08_gr\%C3\%B6\%C3\%9Fte_Stadtprobleme_aus_Sicht _der_Frankfurter.pdf].

Stamatel, Janet P., 2009: Correlates of National-Level Homicide Variation in Post-Communist East-Central Europe. Social Forces 87/3: 1423-1448.

Statistisches Bundesamt/Gesellschaft Sozialwissenschaftlicher Infrastruktureinrichtungen (GESIS-ZUMA)/Wissenschaftszentrum Berling (WZB), 2008: Datenreport 2008: Ein Sozialbericht für die Bundesrepublik Deutschland. Bonn: Bundeszentrale für politische Bildung.

Stedman Jones, Gareth, 1976: Outcast London. A Study in the Relationship Between Classes in Victorian Society. Harmondsworth: Penguin Books.

Stehr, Johannes, 1998: Sagenhafter Alltag. Über die private Aneignung herrschender Moral. Frankfurt/M.: Campus.

Steinberg, Laurence, 2005: Cognitive and Affective Development in Adolescence. Trends in Cognitive Science 9/2: 69-74.

Stelly, Wolfgang/Thomas, Jürgen, 2001: Einmal Verbrecher - Immer Verbrecher? Wiesbaden: Westdeutscher Verlag.

Stelly, Wolfgang/Thomas, Jürgen, 2004: Wege aus schwerer Jugendkriminalität: Eine qualitative Studie zu Hintergründen und Bedingungen einer erfolgreichen Reintegration von mehrfachauffälligen Jungtätern. Tübingen: Universitätsbibliothek Tübingen. Internetquelle: [http://nbn-resolving.de/urn:nbn:de:bsz:21-opus-11254].

Stelly, Wolfgang/Thomas, Jürgen/Kerner, Jürgen, 2003: Verlaufsmuster und Wendepunkte in der Lebensgeschichte. Eine Untersuchung des Einflusses soziobiographischer Merkmale auf sozial abweichende und sozial integrierte Karrieren. Tübingen: Universitaet Tuebingen, TOBIAS-lib.

Stephan, Egon, 1976: Die Stuttgarter Opferbefragung. Eine kriminologisch-viktimologische Analyse zur Erforschung des Dunkelfeldes unter besonderer Berücksichtigung der Einstellung der Bevölkerung zur Kriminalität. Wiesbaden: BKA.

Stoop, Ineke, 2005: The Hunt for the Last Respondent. Nonresponse in Sample Surveys. The Hague: Social and Cultural Planning Office.

Straus, Murray A./Gelles, Richard J./Steinmetz, Suzanne K., 2006: Behind Closed Doors. Violence in the American Family. New Brunswick: Transaction.

Streng, Franz, 2008: Kriminalpolitische Extreme - die Sicht junger Menschen. S. 767-782 in: Görgen, T./Hoffmann-Holland, K./Schneider, H./Stock, J. (Hrsg.), Interdisziplinäre Kriminologie. Festschrift für Arthur Kreuzer zum 70. Geburtstag. Frankfurt/M.: Verlag für Polizeiwissenschaft.

Strüber, Daniel/Lück, Monika/Roth, Gerhard, 2008: Sex, Aggression and Impulse Control. An Integrative Account. Neurocase 14/1: 93-121.

Sutherland, Edwin H., 1945: Is ,,White Collar Crime ${ }^{e e}$ Crime? American Sociological Review 10/2: 132-139.

Sutherland, Edwin H./Cressey, Donald R., 1960: Principles of Criminology. Chicago: J. B. Lippincott.

Svensson, Robert/Oberwittler, Dietrich, 2010: It"es not the Time they Spend, it ${ }^{\text {ec }} \mathrm{s}$ What they Do. The Interaction between Delinquent Friends and Unstructured Routine Activity on Delinquency. Findings from two Countries. Journal of Criminal Justice 38/5: 1006-1014.

Svensson, Robert/Pauwels, Lieven, 2008: Is a "Risky" Lifestyle Always Risky? The Interaction Between Individual Propensity and Lifestyle Risk in Adolescent Offending A Test in Two Urban Samples. Crime \& Delinquency 56/4: 608-624.

Svensson, Robert/Ring, Jonas, 2007: Trends in Self-Reported Youth Crime and Victimization in Sweden, 19952005. Journal of Scandinavian Studies in Criminology and Crime Prevention 8: 185-209. 
Takahashi, Hidehiko/Kato, Motoichiro/Matsuura, Masato/Mobbs, Dean/Suhara, Tetsuya/Okuba, Yoshiro, 2009: When Your Gain Is My Pain and Your Pain Is My Gain: Neural Correlates of Envy and Schadenfreude. Science 323: 937-939.

Taylor, Ralph B., 2001: Breaking Away from Broken Windows. Baltimore Neighborhoods and the Nationwide Fight against Crime, Grime, Fear, and Decline. Boulder, Col.: Westview Press.

Thomas, Jürgen/Stelly, Wolfgang, 2008: Kriminologische Verlaufsforschung zu Jugendkriminalität. Entwicklungen und Befunde. Forensische Psychiatrie, Psychologie, Kriminologie 2/3: 199-206.

Thome, Helmut, 2004: Theoretische Ansätze zur Erklärung langfristiger Gewaltkriminalität seit Beginn der Neuzeit. S. 315-345 in: Heitmeyer, W./Soeffner, H.-G. (Hrsg.), Gewalt. Frankfurt/M.: Suhrkamp.

Thome, Helmut, 2005: Sozialer Wandel und Gewaltkriminalität. Erklärungskonzepte und Methodenprobleme. S. 209-234 in: Heitmeyer, W./Imbusch, P. (Hrsg.), Integrationspotenziale einer modernen Gesellschaft. Wiesbaden: VS - Verlag für Sozialwissenschaften.

Thome, Helmut, 2007: Explaining the Long-Term Trend in Violent Crime. A Heuristic Scheme and some Methodological Considerations. International Journal of Conflict and Violence 1/2: 185-202.

Thome, Helmut/Birkel, Christoph, 2007: Sozialer Wandel und Gewaltkriminalität. Deutschland, England und Schweden im Vergleich, 1950 bis 2000 . Wiesbaden: VS - Verlag für Sozialwissenschaften.

Thornberry, Terence P., 1996: Empirical Support for Interactional Theory: A Review of the Literature. S. 198-235 in: Hawkins, J.D. (Hrsg.), Delinquency and Crime. Current Theories. Cambridge: Cambridge University Press.

Thornberry, Terence P./Krohn, Marvin D./Lizotte, Alan J./Smith, Carolyn A./Tobin, Kimberly, 2003: Gangs and Delinquency in Developmental Perspective. Cmabridge: Cambridge University Press.

Thornberry, Terence P./Lizotte, Alan/Krohn, Marvin D./Farnworth, Margaret/Jang, Sung J., 1994: Delinquent Peers, Beliefs, and Delinquent Behavior. A Longitudinal Test of Interactional Theory. Criminology 32/1: 4783.

Thurman, Quint C., 1989: General Prevention of Tax Evasion. A Factorial Survey Approach. Journal of Quantitative Criminology 5/2: 127-146.

Tillmann, Klaus-Jürgen/Holler-Nowitzki, Birgit/Holtappels, Heinz G./Meier, Ulrich/Popp, Ulrike, 1999: Schülergewalt als Schulproblem. Verursachende Bedingungen, Erscheinungsformen und pädagogische Handlungsperspektiven. Weinheim: Juventa.

Tittle, Charles R., 1983: Social Class and Criminal Behavior. A Critique of the Theoretical Foundation. Social Forces 62/2: 334-358.

Tittle, Charles R./Villemez, Wayne J./Smith, Douglas A., 1978: The Myth of Social Class and Criminality: An Empirical Assessment of the Empirical Evidence. American Sociological Review 43: 643-656.

Tolnay, Stewart E./Beck, E. M., 1995: A Festival of Violence. An Analysis of Southern Lynchings, 1882-1930. Urbana, Ill.: University of Illinois Press.

Tonry, Michael, 2004: Why Aren "t German Penal Policies Harsher and Imprisonment Rates Higher? German Law Journal 5/10: 1187-1206.

Tonry, Michael, (Hrsg.) 2007: Crime, Punishment, and Politics in a Comparative Perspective (Crime and Justice. A Review of Research, Vol. 36). Chicago: Chicago University Press.

Tricomi, Elizabeth/Rangel, Antonio/Camerer, Rangel/O'Doherty, John P., 2010: Neural Evidence for InequalityAverse Social Preferences. Nature 463: 1089-1092.

Trotha, Trutz von, 1982: Zur Entstehung von Jugend. Kölner Zeitschrift für Soziologie und Sozialpsychologie 34/2: 254-277.

Trotha, Trutz von, 2002: Über die Zukunft der Gewalt. Monatsschrift für Kriminologie und Strafrechtsreform 85/5: 349-368.

Tseloni, Andromachi, 2000: Personal Criminal Victimization in the United States. Fixed and Random Effects of Individual and Household Characteristics. Journal of Quantitative Criminology 16/4: 415-442.

Tseloni, Andromachi, 2006: Multilevel Modeling of the Number of Property Crimes: Household and Area Effects. Journal of the Royal Statistical Society, Series A 169/2: 205-233.

Tseloni, Andromachi/Pease, Ken, 2005: Population Inequality. The Case of Repeat Crime Victimization. International Review of Victimology 12: 75-90.

Tseloni, Andromachi/Wittebrood, Karin/Farrell, Graham/Pease, Ken, 2004: Burglary Victimization in England and Wales, the United States and The Netherlands. A Cross-National Comparative Test of Routine Activities and Lifestyle Theories. British Journal of Criminology 44/1: 66-91.

Tyler, Tom R., 1990: Why People Obey the Law. New Haven: Yale University Press.

Tyler, Tom R./Boeckmann, Robert J., 1997: Three Strikes and You are Out, but Why? The Psychology of Public Support for Punishing Rule Breakers. Law Society Review 31/2: 237-266. 
van der Heijden, Peter G. M./van Gils, Ger/Bouts, Jan/Hox, Joop J., 2000: A Comparison of Randomized Response, Computer-Assisted Self-Interview, and Face-to-Face Direct Questioning. Eliciting Sensitive Information in the Context of Welfare and Unemployment Benefit. Sociological Methods Research 28/4: 505-537.

van Dijk, Jan/van Kesteren, John/Smit, Paul, 2007: Criminal Victimization in International Perspective. Key Findings from the 2004-2005 ICVS and EU ICS. Den Haag: WODC.

Vanberg, Viktor, 1993: Rational Choice vs. Adaptive Rule-Following. On the Behavioral Foundations of the Social Sciences. Jahrbuch für Neue Politische Ökonomie 12: 93-110.

Vazsonyi, Alexander T., 2004: The General Theory of Crime in the European Context. Revisiting Hedonic Calculus. S. 337-355 in: Oberwittler, D./Karstedt, S. (Hrsg.), Soziologie der Kriminalität (Sonderheft 43 der Kölner Zeitschrift für Soziologie und Sozialpsychologie). Wiesbaden: VS - Verlag für Sozialwissenschaften.

Vazsonyi, Alexander T./Pickering, Lloyd E./Junger, Marianne/Hessing, Dick, 2001: An Empirical Test of a General Theory of Crime. A Four-Nation Comparative Study of Self-Control and the Prediction of Deviance. Journal of Research in Crime \& Delinquency 38/2: 91-131.

Vidmar, Neil, 2001: Retributive Justice: Its Social Context. S. 291-313 in: Ross, M./Miller, D.T. (Hrsg.), The Justice Motive in Everyday Life. Essays in Honor of Melvin J. Lerner. Cambridge: Cambridge University Press.

Villmow, Bernhard/Stephan, Egon, 1983: Jugendkriminalität in einer Gemeinde: Eine Analyse erfragter Delinquenz und Viktimisierung sowie amtlicher Registrierung. Freiburg i. Br.: edition isucrim.

Voß, Hans-Georg W./Hoffmann, Jens/Wondrak, Isabel (Hrsg.), 2006: Stalking in Deutschland aus Sicht der Betroffenen und Verfolger. Baden-Baden: Nomos.

Wacquant, Loïc/Wilson, William J., 1989: The Cost of Racial and Class Exclusion in the Inner City. Annales of the American Academy of Political and Social Science 501: 8-25.

Waldmann, Peter, 2007: Is There a Culture of Violence in Colombia? Terrorism and Political Violence 19/4: 593609.

Walklate, Sandra, 2007: Imaging the Victim of Crime. Maidenhead: Open University Press.

Wallander, Lisa, 2009: 25 Years of Factorial Surveys in Sociology. A Review. Social Science Quarterly 38/3: 505520.

Walter, Michael, 1999: Von der Kriminalität in den Medien zu einer bedrohlichen Medienkriminalität und Medienkriminologie. DVJJ-Journal 11: 348-254.

Walter, Michael, 2001: Jugendkriminalität. Eine systematische Darstellung [2. Auflage]. Stuttgart: Boorberg.

Walter, Michael/Albrecht, Hans-Jörg/Kania, Harald, 2004: Alltagsvorstellung von Kriminalität. Individuelle und gesellschaftliche Bedeutung von Kriminalitätsbildern für die Lebensgestaltung. Münster: Lit.

Warr, Mark, 2002: Companions in Crime: The Social Aspects of Criminal Conduct. Cambridge: Cambridge University Press.

Weerman, Frank M./Bijleveld, Catrien, 2007: Birds of Different Feathers: School Networks of Serious Delinquent, Minor Delinquent and Non-delinquent Boys and Girls. European Journal of Criminology 4/4: 357-383.

Weerman, Frank M./Smeenk, Wilma H., 2005: Peer Similarity in Delinquency for Different Types of Friends. A Comparison Using Two Measurement Methods. Criminology 43/2: 499-524.

Weigend, Thomas, 2001: Sentencing and Punishment in Germany. S. 188-221 in: Tonry, M./Frase, R.S. (Hrsg.), Sentencing and Sanctions in Western Countries. Oxford: Oxford University Press.

Weitekamp, Elmar G./Kerner, Hans-Jürgen/Stelly, Wolfgang/Thomas, Jürgen, 2000: Desistance from Crime. Life History, Turning Points, and Implications for Theory Construction in Criminology. S. 197-218 in: Karstedt, S./Bussmann, K.-D. (Hrsg.), Social Dynamics of Crime and Crime Control. New Theories for a World in Transition. Oxford: Hart.

West, Donald J./Farrington, David P., 1973: Who Becomes Delinquent? London: Heinemann.

Wetzell, Richard F., 2000: Inventing the Criminal. A History of German Criminology 1880-1945. Chapel Hill: University of North Carolina Press.

Wetzels, Peter, 1996: Krimimalität und Opferleben. Immer öfter das Gleiche? Defizite und Perspektiven repräsentativer Opferbefragungen als Methode empirisch-viktimologischer Forschung in der Kriminologie. Monatsschrift für Kriminologie und Strafrechtsreform 79/1: 1-24.

Wetzels, Peter/Enzmann, Dirk/Mecklenburg, Eberhard/Pfeiffer, Christian, 2001: Jugend und Gewalt. Eine repräsentative Dunkelfeldanalyse in München und acht anderen deutschen Städten. Baden-Baden: Nomos.

White, Jennifer L./Moffitt, Terrie E./Earls, Felton J./Robins, Lee/Silva, Phil A., 1990: How Early Can We Tell? Predictors of Childhood Conduct Disorder and Adolescent Delinquency. Criminology 28/4: 507-527.

Whitman, James Q., 2003: Harsh Justice. Criminal Punishment and the Widening Divide between America and Europe. Oxford: Oxford University Press.

Whitman, James Q., 2005: The Comparative Study of Criminal Punishment. Annual Review of Law and Social Science 1: 17-34. 
Wiener, Martin, 1990: Reconstructing the Criminal. Culture, Law and Poicy in England, 1830-1914. Cambridge: Cambridge University Press.

Wikström, Per-Olof H., 1991: Urban Crime, Criminals, and Victims. New York: Springer.

Wikström, Per-Olof H., 2006: Individuals, Settings, and Acts of Crime. Situational Mechanisms and the Explanation of Crime. S. 61-107 in: Wikström, P.-O.H./Sampson, R.J. (Hrsg.), Crime and Its Explanation: Contexts, Mechanisms and Development. Cambridge: Cambridge University Press.

Wikström, Per-Olof H., 2007a: In Search of Causes and Explanations of Crime. S. 117-140 in: King, R.D./Wincup, E. (Hrsg.), Doing Research on Crime and Justice [2. Auflage]. Oxford: Oxford University Press.

Wikström, Per-Olof H., 2007b: The Social Ecology of Crime. The Role of the Environment in Crime Causation. S. 333-357 in: Schneider, H.J. (Hrsg.), Internationales Handbuch der Kriminologie, Band 1: Grundlagen der Kriminologie. Berlin: de Gruyter.

Wikström, Per-Olof H./Butterworth, David, 2006: Adolescent Crime. Individual Differences and Lifestyle Risks. Cullompton: Willan.

Wikström, Per-Olof H./Sampson, Robert J., 2006: Introduction: Toward a Unified Approach to Crime and its Explanation. S. 1-7 in: Wikström, P.-O.H./Sampson, R.J. (Hrsg.), The Explanation of Crime. Context, Mechanisms, and Development. Cambridge: Cambridge Univerity Press.

Wikström, Per-Olof H./Treiber, Kyle, 2007: The Role of Self-Control in Crime in Causation Beyond Gottfredson and Hirschi's General Theory of Crime. European Journal of Criminology 4/2: 237-264.

Wikström, Per-Olof H./Tseloni, Andromachi/Karlis, Dimitris, 2008: Do People Abide by the Law Because they Fear Getting Caught? (Paper Presented at the European Society of Criminology Conference in Edinburgh 2008). Edinburgh.

Wilmers, Nicola/Enzmann, Dirk/Schaefer, Dagmar/Herbers, Karin/Greve, Werner/Wetzels, Peter, 2002: Jugendliche in Deutschland zur Jahrtausendwende: Gefährlich oder gefährdet? Ergebnisse wiederholter, repräsentativer Dunkelfelduntersuchungen zu Gewalt und Kriminalität im Leben junger Menschen 1998-2000. BadenBaden: Nomos.

Wilson, James Q./Kelling, George E., 1982: The Police and Neighborhood Safety. Broken Windows. Atlantic Monthly 249/March: 29-38 [deutsche Übersetzung 1996: Polizei und Nachbarschaftssicherheit. Zerbrochene Fenster. Kriminologisches Journal 28/2: 121-137].

Wilson, William J., 1987: The Truly Disadvantaged. The Inner City, the Underclass, and Public Policy. Chicago: Chicago University Press.

Wittebrood, Karin/Junger, Marianne, 2002: Trends in Violent Crime. A Comparison Between Police Statistics and Victimization Surveys. Social Indicators Research 59: 153-173.

Wittebrood, Karin/Nieuwbeerta, Paul, 1999: Wages of Sin? The Link between Offenders, Lifestyle and Violent Victimisation. European Journal on Criminal Policy and Research 7/63: 80-.

Wolfgang, Marvin E./Ferracuti, Franco, 1967: The Subculture of Violence. Towards an Integrated Theory in Criminology. Beverly Hills: Sage.

Wolfgang, Marvin E./Figlio, Robert M./Sellin, Thorsten, 1972: Delinquency in a Birth Cohort. Chicago: University of Chicago Press.

Wright, Bradley R. E./Caspi, Avshalom/Moffitt, Terrie E./Paternoster, Ray, 2004: Does the Perceived Risk of Punishment Deter Criminally Prone Individuals? Rational Choice, Self-Control, and Crime. Journal of Research in Crime \& Delinquency 41/2: 180-213.

Wrong, Dennis H., 1961: The Oversocialized Conception of Man in Modern Sociology. American Sociological Review 26/2: 183-193.

Zauberman, Renée (Hrsg.), 2008: Victimization and Insecurity in Europe: A Review of Surveys and their Use. Brüssel: VUB Press.

Zauberman, Renée (Hrsg.), 2009: Self-Reported Crime and Deviance Studies in Europe: Current State of Knowledge and Review of Use. Brüssel: VUB Press.

Zimring, Franklin E., 2004: The Contradictions of American Capital Punishment. Oxford: Oxford University Press.

Zuckermann, Marvin, 1979: Sensation Seeking. Beyond the Optimal Level of Arousal. Hillsdale, N.J.: Erlbaum. 